\title{
Cavity magnon-polaritons in cuprate parent compounds
}

\author{
Jonathan B. Curtis $\odot,{ }^{1,2,{ }^{*}}$ Andrey Grankin,${ }^{3}$ Nicholas R. Poniatowski, ${ }^{2}$ Victor M. Galitski, ${ }^{3}$ \\ Prineha Narang ${ }^{\circ},{ }^{1,2}$ and Eugene Demler ${ }^{2,4}$ \\ ${ }^{1}$ John A. Paulson School of Applied Sciences and Engineering, Harvard University, Cambridge, Massachusetts 02138, USA \\ ${ }^{2}$ Department of Physics, Harvard University, Cambridge, Massachusetts 02138, USA \\ ${ }^{3}$ Joint Quantum Institute, Department of Physics, University of Maryland, College Park, Maryland 20742, USA \\ ${ }^{4}$ Institute for Theoretical Physics, ETH Zurich, 8093 Zurich, Switzerland
}

(Received 8 August 2021; revised 9 December 2021; accepted 15 December 2021; published 10 February 2022)

\begin{abstract}
Cavity control of quantum matter may offer new ways to study and manipulate many-body systems. A particularly appealing idea is to use cavities to enhance superconductivity, especially in unconventional or high- $T_{c}$ systems. Motivated by this, we propose a scheme for coupling terahertz resonators to the antiferromagnetic fluctuations in a cuprate parent compound, which are believed to provide the glue for Cooper pairs in the superconducting phase. First, we derive the interaction between magnon excitations of the Neél order and polar phonons associated with the planar oxygens. This mode also couples to the cavity electric field, and in the presence of spin-orbit interactions mediates a linear coupling between the cavity and magnons, forming hybridized magnon-polaritons. This hybridization vanishes linearly with photon momentum, implying the need for near-field optical methods, which we analyze within a simple model. We then derive a higher-order coupling between the cavity and magnons, which is only present in bilayer systems, but does not rely on spin-orbit coupling. This interaction is found to be large, but only couples to the bimagnon operator. As a result, we find a strong, but heavily damped, bimagnon-cavity interaction which produces highly asymmetric cavity line shapes in the strong-coupling regime. To conclude, we outline several interesting extensions of our theory, including applications to carrier-doped cuprates and other strongly correlated systems with terahertz-scale magnetic excitations.
\end{abstract}

DOI: 10.1103/PhysRevResearch.4.013101

\section{INTRODUCTION}

Using light to control the properties of quantum materials not only holds the potential to realize new and interesting quantum many-body phases [1-16] but may also hold the key to create novel devices and functionalities [17-29]. In most cases, this optical control is achieved by externally applying intense electromagnetic radiation to the system in question [30-59]. Recently, however, an appealing alternative route has been put forward which bypasses the need for intense external radiation. Instead, one may attempt to use resonantly coupled electromagnetic cavities to custom tailor the properties of the electromagnetic vacuum fluctuations directly [14,27,28,60-86].

A potentially powerful application of this approach is to use cavities to control antiferromagnetic correlations in a high- $T_{c}$ cuprate superconductor. These correlations are believed to underlie many of the exotic, and potentially useful, aspects of the unconventional high- $T_{c}$ superconductivity in these materials [87-91]. It therefore stands to reason that

\footnotetext{
*jcurtis@ seas.harvard.edu

Published by the American Physical Society under the terms of the Creative Commons Attribution 4.0 International license. Further distribution of this work must maintain attribution to the author(s) and the published article's title, journal citation, and DOI.
}

the ability to optically manipulate, and ultimately enhance, superconductivity in these materials [20,30,43,72,92-95] is extremely appealing from both a theoretical and practical perspective, and may pave the way toward realizing room-temperature superconductivity - a holy grail of modern condensed matter and material science.

The concept of using cavities to manipulate superconductivity has already been put forward in the context of electron-phonon systems $[61,67,96]$. In this case, it has been proposed that strongly coupling resonant cavities to infrared-active optical phonons [97-100] may offer a way to manipulate the pairing between electrons in conventional superconductors. While indeed this may be a promising avenue [79] toward achieving cavity-enhanced superconductivity, these systems still remain fundamentally limited by the relatively weak coupling strengths afforded by electronphonon interaction. In contrast, while the exact mechanism responsible for superconductivity in cuprates is still unclear, it is widely believed to be driven by interactions of electrons with spin fluctuations rather than phonons [87-89,101-118]. As a result, these systems have a higher ceiling for potential enhancement effects. At the same time, it is also less clear how cavities may be used to modify these spin fluctuations.

Motivated by these considerations, we propose a scheme for realizing strong coupling of a resonant cavity to the spin fluctuations in an antiferromagnetic cuprate parent compound. Previously, it has been established that optical methods can be used to manipulate magnons in antiferromagnets, ei- 


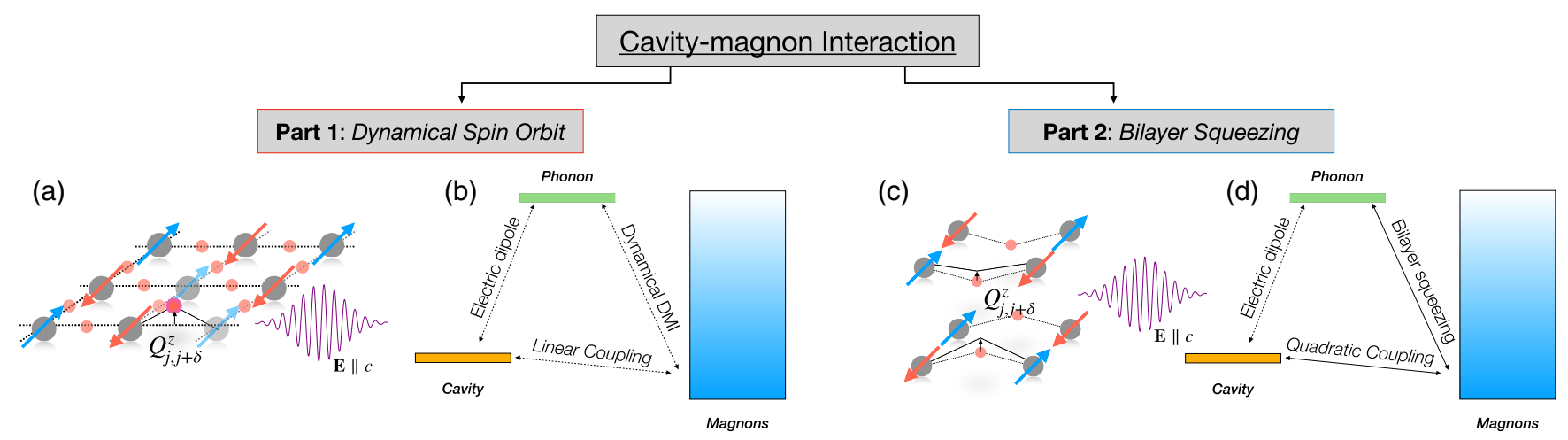

FIG. 1. Overview of our proposal for forming cavity magnon-polaritons in insulating cuprates. The paper is structured into two parts. In part one (Sec. IV), we study a mechanism based on the dynamical Dzyaloshinskii-Moriya interaction. This interaction originates from spin-orbit effects, and the relevant displacement of the phonon mode is shown in (a). We illustrate the energy scales and interaction mechanisms for the cavity-phonon and magnon-phonon interactions, which results in an effective linear magnon-cavity coupling, shown in (b). In part two (Sec. V), we consider a mechanism which is based on a bilayer-squeezing interaction. This interaction doesn't require spin-orbit effects and involves a bilayer-odd perturbation to the scalar exchange. The relevant phonon displacement is shown in (c). We illustrate the energy scales and interaction mechanisms for the cavity-phonon and magnon-phonon interactions, which results in an effective quadratic magnon-cavity coupling, shown in (d).

ther through Raman processes [119-126] or ultrafast optical methods [31,32,57,93,124,127-133]. However, most of these protocols are difficult to adapt to the cavity setting due to the fact that they essentially rely on nonlinear or strongly offresonant processes [74]. Unlike their ferromagnetic counterparts, which reside at $\mathrm{GHz}$ frequencies [134-137], magnons in an antiferromagnetic typically reside in the $1-10 \mathrm{THz}$ regime, owing to their larger superexchange energy scales [138]. This presents additional challenges due to the notorious difficulties in terahertz engineering, which are only recently being overcome [27,28,75,77-79,97,99,139-151]. In this paper, we theoretically address these challenges, thereby taking the first crucial step toward achieving cavity control of high-temperature superconductivity in cuprates.

This leads us to our central proposal of using terahertz resonators to strongly couple to the magnons in a cuprate system, which is schematically depicted in Fig. 1. Specifically, we propose two suitable microscopic mechanisms by which magnons in the insulating cuprate may be coupled to cavity photons, which are organized into two main parts of this paper. Both of these employ an infrared-active phonon mode [57] as an intermediary between the electric field of the cavity and the electron spins. In the first scenario, discussed in part one, we examine how in the presence of spin-orbit coupling [66,152-159] this phonon mode can lead to a linear coupling between the cavity photons and spin-waves [23,160-168]. The relevant displacement of the phonon mode and how it couples to the cavity and magnons is illustrated in Figs. 1(a) and 1(b), respectively. Our key finding is that this generically leads to a linear effective magnetoelectric coupling, but in the absence of inversion-symmetry breaking [169] this coupling vanishes in the far-field limit and the magnons decouple from the electric field. While this poses a problem for conventional terahertz optics, since terahertz resonators are localized well within the subwavelength regime, this will not fundamentally impede the coupling to a resonant cavity. Inspired by recent advances in terahertz near-field optics
[141,144,146,148,170-177], we explore a particular model in which a strong near-field coupling is realized.

In the second scenario, discussed in part two, we examine how the same infrared-active phonon mode may couple to the scalar spin-exchange in a bilayer cuprate. Due to a buckling distortion in equilibrium, in a bilayer the planar bonds are not linear and therefore there may be a linear coupling to a polar mode $[114,178]$. The relevant displacement of the phonon mode in this case and how it couples to the cavity and magnons is illustrated in Figs. 1(c) amd 1(d) respectively. This coupling yields an effective magnetoelectric coupling which is linear in electric field but quadratic in the spin waves. While this coupling doesn't lead to the formation of polaritons, we show that, nevertheless, this does lead to a strong coupling between the photons and cavity, and may be promising in the future since it more naturally allows for controlling the correlations which are moderated by the spin-waves [67,179-184]. Both of these processes are argued to be within the reach of current experiments. A summary of the main results, and which section they are discussed in, is encapsulated in Table I.

TABLE I. Summary of the coupling schemes considered in this paper and the section where they are discussed. For each, we have indicated the structure of the system (monolayer versus bilayer) considered, the physical mechanism underlying the coupling (spin-orbit interaction versus buckling modulation), the order of magnonoperator coupled to (linear coupling to single magnon operator or quadratic coupling to bimagnon operator), and whether a near-field or conventional cavity is needed for experimental detection.

\begin{tabular}{llll}
\hline \hline Section & IV A & IV C & \multicolumn{1}{c}{$\mathrm{V}$} \\
\hline Structure & monolayer & bilayer & bilayer \\
Mechanism & spin-orbit & spin-orbit & buckling \\
Order & linear & linear & quadratic \\
Cavity type & near field & near field & conventional \\
\hline \hline
\end{tabular}


The remainder of this paper is structured as follows: In Sec. II, we review the equilibrium structure of the model cuprate system and its interactions with lattice vibrations and spin-orbit effects. In Sec. III, we briefly review the equilibrium properties of the model described in the previous section. Next, in Sec. IV we focus our attention on a resulting linear magnetoelectric coupling and show how it can lead to hybridized cavity magnon-polaritons. After examining the linear coupling, we proceed to Sec. V, where we examine a quadratic coupling between the cavity photons and bimagnons also present in our model, and argue it can also give rise to strong-coupling signatures between the cavity-photons and bimagnons. Finally, we conclude with a discussion in Sec. VI, wherein we outline future interesting directions and applications of our paper.

\section{MODEL}

We begin with the nature of spin-orbit coupling in the cuprates. We will mainly focus on modeling bilayer cuprate systems, as realized by the $\mathrm{YBa}_{2} \mathrm{Cu}_{3} \mathrm{O}_{6}$ (YBCO) family of compounds. However, it is often qualitatively similar, but technically simpler, to consider a model system which consists of a single monolayer with easy-plane anisotropy. The details of the derivation of the easy-plane toy model will be relegated to Appendix B, though it will also straightforwardly follow as a limiting case of decoupled bilayers.

This section is devoted to deriving the relevant spin model and its coupling to the cavity electric field, summarized as

$$
\hat{H}_{\mathrm{tot}}=\hat{H}_{\mathrm{sp}}+\hat{H}_{\mathrm{int}} \text {. }
$$

First, we will derive the appropriate spin-model, which can be found later in Eq. (4). We will then derive via a spinphonon coupling the magnon-cavity interaction Hamiltonian $H_{\text {int }}$, which can be found later in Eq. (11). First, we consider the effects of spin-orbit coupling and derive the stand-alone spin Hamiltonian.

\section{A. Spin-orbit coupling}

We will first focus on the case of a bilayer system, such as YBCO. Importantly, in the compound YBCO there are two copper oxide planes in each unit cell which are related to each other by horizontal mirror plane symmetry. Therefore, overall inversion symmetry is preserved but can still act nontrivially on each layer [185].

To lowest order, we expect that at half filling the lowenergy Hamiltonian for the system should roughly map onto a nearest-neighbor Heisenberg model with weak interlayer coupling [153] of the form

$$
\hat{H}_{0}=\sum_{\ell} \sum_{\langle j, k\rangle} J_{0} \hat{\boldsymbol{S}}_{j \ell} \cdot \hat{\boldsymbol{S}}_{k \ell}+\sum_{j} J_{u d} \hat{\boldsymbol{S}}_{j u} \cdot \hat{\boldsymbol{S}}_{j d},
$$

where the vector operator $\hat{\boldsymbol{S}}_{j \ell}$ describes the spin- $\frac{1}{2}$ moment located at site $\mathbf{R}_{j}$ of layer $\ell=u, d$, and $\langle j, k\rangle$ indicates that $j$ and $k$ are nearest-neighbor sites within the $a b$ plane. The interlayer coupling $J_{u d}$ involves the hopping along the $c$ axis and is typically two orders of magnitude smaller than the intralayer processes [153].
So far, this model neglects spin-orbit effects which, though small, are important at the terahertz scale, as they set the scale for the magnon gap $[138,153]$. To obtain the spin-orbit coupling corrections to superexchange [186-188], we start with a single-band bilayer Hubbard model with spin-orbit coupled hopping $i \lambda \cdot \sigma$ [189]. This is then downfolded, in the usual way, onto the ground-state spin manifold. Since it is not relevant for our discussion going forward, we relegate the actual derivation of the corresponding spin model to Appendix A. Ultimately, the important parameter reflecting the spin-orbit coupling is $\lambda_{\delta \ell}$, which depends on the bond direction and layer index. In particular, time-reversal symmetry constrains $\lambda$ to be real and Hermiticity requires $\lambda_{\delta}=-\lambda_{-\delta}$. In a monolayer system, inversion symmetry then requires $\lambda_{\delta}=\lambda_{-\delta}$, and thus the spin-orbit coupling must vanish. However, the crucial observation is that in the case of YBCO, inversion symmetry only constrains the spin-orbit coupled hopping to obey $\lambda_{-\delta u}=\lambda_{\delta d}$, which allows for nonzero spin-orbit coupling on each layer. In this paper, we consider the effect of a Rashba spin-orbit texture, with

$$
\lambda_{\delta \ell}=(-1)^{\ell} \lambda \mathbf{e}_{z} \times \delta .
$$

Following Ref. [159], we take as a rough estimate the spin-orbit interaction $\lambda \sim 10 \mathrm{meV}$. For the isotropic superexchange, as a rough estimate, we will assume typical values of $t \sim 400 \mathrm{meV}$ and $U \sim 4.2 \mathrm{eV}$ [87].

Microscopically, this Rashba spin-orbit coupling emerges naturally if one notes that in a bilayer system like YBCO, local crystal field gradients on each layer cause a uniform buckling of the planar oxygens in the copper oxide planes toward the interior of the bilayers [66,152-154,157-159,178,189]. This displaces the oxygen ligands by about $0.22 \AA$ from the copper oxide plane, leading to a nonlinear bond angle and, as a result, an unquenched Dzyaloshinskii-Moriya (DM) interaction may exist on each bond. It is also worth commenting that recently, effects of similar spin-orbit couplings have been seen in the dynamics of charge carriers in hole-doped cuprates YBCO $[159,190]$ and $\mathrm{Bi}-2212[66,191]$, reinforcing this theoretical model.

It is now straightforward to obtain the spin-orbit corrections to Hamiltonian Eq. (2) due to spin-orbit coupling in Eq. (3). As we derive in Appendix A, one arrives at the lowenergy Rashba bilayer spin model, which replaces Eq. (2), of

$$
\begin{aligned}
\hat{H}_{\mathrm{sp}}= & \sum_{j \in A \ell \delta} J_{0} \hat{S}_{j \ell} \cdot \hat{S}_{j+\delta \ell}+\Gamma_{\delta}^{a b} \hat{S}_{j \ell}^{a} \hat{S}_{j+\delta \ell}^{b}+\mathbf{D}_{\boldsymbol{\delta} \ell} \cdot \hat{\mathbf{S}}_{j \ell} \times \hat{\mathbf{S}}_{j+\delta \ell} \\
& +\sum_{j} J_{u d} \hat{\mathbf{S}}_{j u} \cdot \hat{\mathbf{S}}_{j d}
\end{aligned}
$$

with intralayer exchange tensors

$$
\begin{aligned}
J_{0} & =4 \frac{t^{2}-\lambda^{2}}{U}, \\
\Gamma_{\delta}^{a b} & \equiv \Gamma\left(\mathbf{e}_{z} \times \boldsymbol{\delta}\right)_{a}\left(\mathbf{e}_{z} \times \boldsymbol{\delta}\right)_{b}=\frac{8 \lambda^{2}}{U}\left(\mathbf{e}_{z} \times \boldsymbol{\delta}\right)_{a}\left(\mathbf{e}_{z} \times \boldsymbol{\delta}\right)_{b}, \\
\mathbf{D}_{\boldsymbol{\delta} \ell} & \equiv D_{\ell} \mathbf{e}_{z} \times \boldsymbol{\delta}=-(-1)^{\ell} \frac{8 t \lambda}{U} \mathbf{e}_{z} \times \boldsymbol{\delta} .
\end{aligned}
$$


To avoid double counting, we have implemented the sum over bonds as a sum over sites in the $A$ sublattice, and a sum over its neighbors (which reside in the $B$ sublattice).

We are now interested in coupling this spin system to the optical field of an electromagnetic cavity. We will outline a few possible mechanisms to achieve this coupling, all of which exploit electron-phonon interactions. Within the context of ultrafast optics, phonomagnetic interactions are by now a well-established way of coupling terahertz optical fields to electronic spins $[31,34,36,49,161,192-196]$. The combination of a relatively large electric dipole matrix element and strong electron-phonon interaction [197] at the appropriate energy scale also makes this a promising route for engineering linear coupling of spins to the cavity electromagnetic field. However, before proceeding, it is worth briefly commenting that other mechanisms, such as direct magnetic-dipole coupling $\quad[57,129-133,137,142,143,145,147,149,198-202]$ or more complicated electric-dipole couplings $[74,119,127,128,203-210]$ may also be potentially fruitful but lie outside the scope of this paper and will be discussed in future works. In the next subsection, we will consider one particular phonon mode and show how it can be used to obtain a coupling between the spin system and the cavity mode.

\section{B. Polar phonon coupling}

Typically, a polar phonon mode cannot couple linearly to the spin degrees of freedom in a centrosymmetric system due to inversion symmetry. We will investigate two ways around this obstruction. The first is through spin-orbit interactions [160-168]. This mechanism is generic to both the monolayer and bilayer systems and generates both linear and quadratic couplings. The second mechanism is specific to the bilayer system and only generates quadratic couplings, but is expected to be much stronger than the spin-orbit interaction [211]. This interaction can be understood as arising from linearizing the standard biquadratic $\sim Q^{2} \mathbf{S} \cdot \mathbf{S}$ coupling of the polar mode around the buckled equilibrium structure, which is allowed only in the bilayer system.

For the purpose of specificity, we will focus on the well-known infrared-active $B_{1 u}$ mode which is located at a frequency of roughly $9 \mathrm{THz} \quad\left(41 \mathrm{meV}, 320 \mathrm{~cm}^{-1}\right)$ $[30,51,93,122,123,178,212-222]$ in YBCO. This mode corresponds to a uniform, in-phase $c$-axis vibration of all of the planar oxygen atoms in both bilayers.

We will first write down the stand-alone phonon Hamiltonian (including the coupling to the electric field) $H_{\mathrm{ph}}$ before going on to derive the relevant spin-phonon Hamiltonian $H_{\mathrm{sp}-\mathrm{ph}}$. The bare phonon dynamics is modeled by the Hamiltonian $H_{\mathrm{ph}}$, given as

$$
\begin{aligned}
\hat{H}_{\mathrm{ph}}= & \sum_{j \in A, \delta} \frac{1}{2 M_{\mathrm{ph}}} \hat{P}_{j, j+\delta}^{2}+\frac{1}{2} M_{\mathrm{ph}} \Omega_{\mathrm{ph}}^{2} \hat{Q}_{j, j+\delta}^{2} \\
& -Z_{\mathrm{ph}} e \hat{E}_{j, j+\delta}^{z} \hat{Q}_{j, j+\delta},
\end{aligned}
$$

where to avoid double counting we have instituted the sum over all bonds by introducing the two sublattices $A$ and $B$ and summing over each site in $A$ and all of its neighboring sites at $j+\delta$, which are in sublattice $B$. Here $\hat{Q}_{j, j+\delta}$ describes the phonon displacement of the oxygen residing on the bond connecting sites $j \in A$ and $j+\delta \in B$, and $\hat{P}_{j, j+\delta}$ is the corresponding canonically conjugate momentum. In addition to the resonance frequency of $\Omega_{\mathrm{ph}} \sim 41 \mathrm{meV}$, we also have introduced the phonon effective mass $M_{\mathrm{ph}} \sim 8 M_{\mathrm{O}}$, and Born effective charge $Z_{\mathrm{ph}} \sim 8 Z_{\mathrm{O}}$, with $Z_{\mathrm{O}}, M_{\mathrm{O}}$ the effective charge and mass of one of the planar oxygens.

To obtain the spin-phonon coupling Hamiltonian $H_{\text {sp-ph }}$, we could once again begin with an electron-phonon interaction and then downfold this to obtain a spin-phonon coupling. As before, the actual details of the electronic model are not relevant and the form of the couplings could, in principle, largely be surmised on symmetry grounds alone. As a result, the details of this procedure are again relegated to Appendix A.

Then the important parameters are essentially $\alpha$, which is the phonon-induced change in the spin-isotropic hopping $t$, and $\kappa$, which is the phonon-induced change in the spin-orbit coupled hopping $\lambda$ [223]. In a bilayer structure, both of these are nonzero, while in a monolayer system only $\kappa$ is present. The resulting spin-phonon coupling is then found in the bilayer system to be

$$
\begin{aligned}
\hat{H}_{\mathrm{sp}-\mathrm{ph}}= & \sum_{j \ell \delta} \frac{8 t \alpha}{U}(-1)^{\ell} \hat{Q}_{j, j+\delta} \hat{\mathbf{S}}_{j \ell} \cdot \hat{\mathbf{S}}_{j+\delta \ell} \\
& +\frac{8 t \kappa}{U} \hat{Q}_{j, j+\delta}\left(\mathbf{e}_{z} \times \boldsymbol{\delta}\right) \cdot \hat{\mathbf{S}}_{j \ell} \times \hat{\mathbf{S}}_{j+\delta \ell} .
\end{aligned}
$$

We now perform a rough estimation of the sizes of the electron-phonon coupling constants, based on the known equilibrium properties. To estimate the spin-orbit parameter $\kappa$, we observe that in equilibrium the spin-orbit exchange in the bilayer system is roughly $\lambda \sim 10 \mathrm{meV}$ [159]. For small displacements, this should be roughly proportional to the equilibrium oxygen displacement $Q_{\mathrm{O}} \sim 0.22 \AA$. We therefore extrapolate that the constant $\kappa \sim \lambda / Q_{\mathrm{O}} \sim 45 \mathrm{meV} \AA^{-1}$. This then yields a spin-phonon coupling constant of $8 t \kappa / U \sim$ $34 \mathrm{meV}^{-1}$.

The parameter $\alpha$ is more difficult to estimate accurately. We will use the rough estimates provided in Refs. [114,178], where it is estimated that $2 \alpha / t \sim 5 / 0.22 \AA$ and therefore this coupling can be quite large, with effective spin-phonon coupling constant $8 \alpha t / U \sim 2.7 \mathrm{eV} \AA^{-1}$.

If we approximate the phonon frequency to be large compared to the frequency of the spin fluctuations of interest, we can treat the phonon in the Born-Oppenheimer approximation. This is acceptable for magnetic zone-center spin waves, which have frequencies of order $2-3 \mathrm{meV}$, however, in general, the magnon bandwidth is still much larger than the phonon resonance frequency and, in this case, a more involved treatment of the full spin-phonon-photon model is needed. Since the interaction with the cavity is dominated by the zone-center magnons (see discussion in Sec. IV B), we proceed to make the Born-Oppenheimer approximation obtain a direct magnetoelectric coupling.

The details of this procedure can be found in Appendix D, where we use an effective action approach to integrate out the phonon mode. We now roughly outline the intuition. 
The term of interest in this paper is the resulting coupling between the electric field and the magnons, which we obtain in the static limit by assuming the phonon mode is driven by the electric field via the standard response kernel

$$
Q^{z}(\omega)=\frac{Z_{\mathrm{ph}} e}{M_{\mathrm{ph}}\left(\Omega_{T}^{2}-\omega^{2}\right)} E^{z}(\omega) \sim \frac{Z_{\mathrm{ph}} e}{M_{\mathrm{ph}} \Omega_{T}^{2}} E^{z}(\omega),
$$

with the last relation holding at low frequencies as compared to the phonon resonance.

This is replaced into the spin-phonon coupling Hamiltonian $H_{\mathrm{sp}-\mathrm{ph}}$, yielding the effective magnetoelectric interaction Hamiltonian we use in this paper. In general, we will also find a number of other terms which result from a more careful treatment, even in the adiabatic limit. These include effective four-body spin interactions and the regular dielectric response of the phonons, both of which we neglect in this paper since they don't immediately lead to a cavity-magnon interaction and are present regardless of the cavity. Additionally, near the resonance one cannot justifiably integrate out the phonon mode, and the three coupled systems (the cavity, magnons, and phonons) ought to all be treated on equal footing.

This is both a very interesting and important direction for future work. However, since the focus of this paper is solely on the cavity-magnon coupling aspect, we proceed onward with only the magnetoelectric interaction kept. We also comment that one could, on symmetry grounds, essentially posit the form of the magnetoelectric interaction from the outset, as is done, for instance, in Ref. [127]. We find the result

$$
\begin{aligned}
H_{\text {int }}= & \sum_{j \ell \delta} g^{\prime}(-1)^{\ell} \hat{E}_{j, j+\delta}^{z} \hat{\mathbf{S}}_{j \ell} \cdot \hat{\mathbf{S}}_{j+\delta \ell} \\
& +g \hat{E}_{j, j+\delta}^{z}\left(\mathbf{e}_{z} \times \boldsymbol{\delta}\right) \cdot \hat{\mathbf{S}}_{j \ell} \times \hat{\mathbf{S}}_{j+\delta \ell} .
\end{aligned}
$$

The magnetoelectric coupling constants $g, g^{\prime}$ are given by

$$
\begin{aligned}
g & =\frac{Z_{\mathrm{ph}} e}{M_{\mathrm{ph}} \Omega_{\mathrm{ph}}^{2}} \frac{8 t \kappa}{U}, \\
g^{\prime} & =\frac{Z_{\mathrm{ph}} e}{M_{\mathrm{ph}} \Omega_{\mathrm{ph}}^{2}} \frac{8 t \alpha}{U} .
\end{aligned}
$$

Using a rough crystal-field approximation, we estimate that $Z_{\mathrm{ph}}=2 e, M_{\mathrm{ph}}=8 \mathrm{u}$, and $\Omega_{\mathrm{ph}}=10 \mathrm{THz}$. Therefore, the static polarizability of the phonon mode in question is $Z_{\mathrm{ph}} e /\left(M_{\mathrm{ph}} \Omega_{\mathrm{ph}}^{2}\right) \sim 1.3 \times 10^{-4} \AA / \mathrm{kV} \mathrm{cm}^{-1}$. This corresponds to a value of $g \sim 4.4 \times 10^{-3} \mathrm{meV} / \mathrm{kV} \mathrm{cm}^{-1}$. An electric field strength on the order of $E^{z} \sim 100 \mathrm{kV} \mathrm{cm}^{-1}$ would then lead to a change in the strength of the DM interaction by an amount of order $0.4 \mathrm{meV}$ or roughly $5 \%$ of the equilibrium value. We also find that $g^{\prime}=(\alpha / \kappa) g \sim 80 g$ can be quite large, though there is a notable degree of uncertainty in the size of our estimate of the coupling constant $\alpha$.

Altogether, we are now tasked with studying the dynamics of the full effective Hamiltonian, as claimed above, of

$$
\begin{aligned}
\hat{H}_{\mathrm{tot}}= & \hat{H}_{\mathrm{sp}}+\hat{H}_{\mathrm{int}}=\sum_{j \in A \ell \delta} J_{\delta}^{a b} \hat{S}_{j \ell}^{a} \hat{S}_{j+\delta \ell}^{b} \\
& +\mathbf{D}_{\delta \ell} \cdot \hat{\mathbf{S}}_{j \ell} \times \hat{\mathbf{S}}_{j+\delta \ell}+\sum_{j \in A, B} J_{u d} \hat{\mathbf{S}}_{j u} \cdot \hat{\mathbf{S}}_{j d}
\end{aligned}
$$

$$
\begin{aligned}
& +\sum_{j \in A \ell \delta} g \hat{E}_{j, j+\delta}^{z}\left(\mathbf{e}_{z} \times \boldsymbol{\delta}\right) \cdot \hat{\mathbf{S}}_{j \ell} \times \hat{\mathbf{S}}_{j+\delta \ell} \\
& +g^{\prime}(-1)^{\ell} \hat{E}_{j, j+\delta}^{z} \hat{\mathbf{S}}_{j \ell} \cdot \hat{\mathbf{S}}_{j+\delta \ell} .
\end{aligned}
$$

Here we have included the weak interlayer superexchange $J_{u d}$, which is known to play a crucial role in stabilizing the observed Néel order [153]. Furthermore, in the absence of spin-orbit considerations, it is important to include an interlayer coupling term, otherwise for a uniform $E^{z}$ the dynamics of the $g^{\prime}$ interaction will actually be trivial since it will commute with the unperturbed dynamics. We have also implemented the convention that the sum over intralayer bonds is carried out by introducing the $A$ and $B$ sublattices and summing over sites only in the $A$ sublattice, along with each nearest-neighbor bond of that site (which is a site lying in the $B$ sublattice). Before examining the consequences of the magnetoelectric coupling, we will review the equilibrium spin-wave dynamics of Hamiltonian Eq. (11). In addition, since the full spin dynamics of Hamiltonian Eq. (11) is rather complicated, we will also analyze a stand-in easy-plane model which, when possible, we will use to simplify the analysis. This Hamiltonian can be found below in Eq. (12) and essentially captures the most important aspect of the full spin-orbit interaction above, which is that it favors in-plane Neel order.

\section{EQUILIBRIUM SPIN-WAVE SPECTRUM}

We now quickly review the equilibrium spin-wave dispersion relations. We will first study the dynamics of the simplified monolayer easy-plane antiferromagnet before proceeding on to study the full bilayer Rashba model Eq. (11), which will end up bearing a number of similarities to the monolayer toy model.

\section{A. Easy-plane toy model}

To gain intuition, we begin by studying a simplified model of a single copper oxide plane with Hamiltonian

$$
H_{\mathrm{EP}}=\sum_{j \in A, \delta} J_{0} \hat{\mathbf{S}}_{j} \cdot \hat{\mathbf{S}}_{j+\delta}-\Gamma \hat{S}_{j}^{z} \hat{S}_{j+\delta}^{z},
$$

which has a uniaxial anisotropy $\Gamma>0$. In this case, we take estimates for the parameters of $J_{0}=150 \mathrm{meV}$ and $\Gamma=$ $0.01 \mathrm{meV}$, which gives a spin-wave gap of order of $3.4 \mathrm{meV}$ for the gapped mode. We emphasize again, this model is meant to serve as a conceptual aid in understanding the behavior of the more complicated mode given above in Eq. (11). The mean-field ground state of this system has Néel order which lies in the $a-b$ plane; we take it to lie at an angle $\theta$ with respect to the crystalline $a$ axis. We now pass to a right-handed coordinate system, with unit vectors $\mathbf{e}_{1}, \mathbf{e}_{2}, \mathbf{e}_{3}$, such that $\mathbf{e}_{3}$ is aligned with the Neel order and $\mathbf{e}_{1}=\mathbf{e}_{z}$. For details, we refer the interested reader to Appendix B.

We now perform the standard Holstein-Primakoff expansion for the spin operators in this coordinate system. These are compactly collected into a four-component bosonic Nambu spinor $\Psi_{\mathbf{p}}=\left(a_{\mathbf{p}}, b_{\mathbf{p}}, a_{-\mathbf{p}}^{\dagger}, b_{-\mathbf{p}}^{\dagger}\right)^{T}$. We also introduce the Nambu-space Pauli-matrices $\tau$ and sublattice-space Pauli matrices $\zeta$. Expanding the spin-wave Hamiltonian to quadratic 


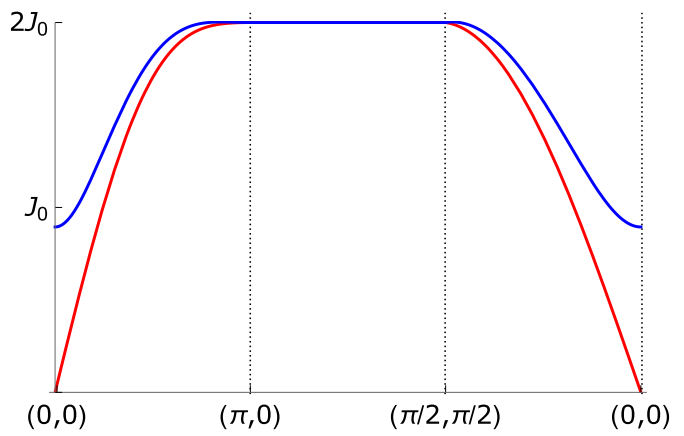

FIG. 2. Dispersion of the two spin-wave bands for easy plane toy model with dispersion given in Eq. (15). This is shown for an exaggerated anisotropy of $\Gamma \sim .1 J_{0}$, which results in a large spinwave gap for one of the modes (blue, upper). In reality, the anisotropy is expected to be much smaller, since the gap scales with the square root of the anisotropy.

order we then find

$$
H_{\mathrm{EP}}=\frac{1}{2} \sum_{\mathbf{p}} \Psi_{\mathbf{p}}^{\dagger}\left[2 J_{0}-\Gamma \gamma_{\mathbf{p}}^{s} \zeta_{1}+\left(2 J_{0}-\Gamma\right) \gamma_{\mathbf{p}}^{s} \zeta_{1} \tau_{1}\right] \Psi_{\mathbf{p}},
$$

where $\gamma_{\mathbf{p}}^{s}$ is the cubic harmonic of (extended) $s$-wave symmetry

$$
\gamma_{\mathbf{p}}^{s}=\frac{1}{2}\left(\cos p_{x}+\cos p_{y}\right) .
$$

This can be diagonalized by standard unitary and Bogoliubov transformations (see Appendix B). We find two dispersion relations indexed by their sublattice quantum number $\zeta= \pm 1$ :

$$
\Omega_{\mathbf{p}, \zeta}=\sqrt{\left(2 J_{0}-\zeta \Gamma \gamma_{\mathbf{p}}^{s}\right)^{2}-\left(2 J_{0}-\Gamma\right)^{2}\left(\gamma_{\mathbf{p}}^{s}\right)^{2}} .
$$

This dispersion relation is plotted in Fig. 2. The $\zeta=-1$ mode is gapped to a frequency $\sqrt{8 J_{0} \Gamma}$, while the $\zeta=+1$ mode remains a gapless Goldstone mode, with spin-wave velocity $v \sim \sqrt{2 J_{0}\left(2 J_{0}-\Gamma\right)}$. In the absence of interlayer exchange or further in-plane anisotropies, true long-range Néel order is then destroyed at finite temperatures in accordance with the Mermin-Wagner theorem.

\section{B. Full bilayer Rashba model}

We now proceed to consider the full bilayer Hamiltonian of Eq. (4). Following Ref. [153], we make the ansatz that the ground state is a Néel order which lies in the $a-b$ plane (again at angle $\theta$ with respect to the $a$ axis) and that is opposite on the two layers. The motivation for the easy-plane Néel order is that all anisotropies in the Hamiltonian Eq. (11) lie within the $a-b$ plane. Specifically, the Rashba spin-orbit coupling results in an easy-axis anisotropy which favors Néel ordering along the $b$ axis for $x$-oriented bonds, and $a$ axis ordering along the $y$-oriented bonds. While this is clearly frustrated between ordering along $a$ or $b$, it is clear that no anisotropies favor $c$-axis order, and therefore the Néel order in the ground state lies in the $a-b$ plane [224].

Since the Néel order is assumed to lie in the $a-b$ plane, we adopt the same axes as in the easy-plane calculation, and again expand to quadratic order in the Holstein-Primakoff bosons. For details of the expansion, see Appendix C. Unlike the previous easy-plane case, there are now two layers and therefore the spin-wave operators now carry a bilayer quantum number. This brings the bosonic Nambu spinor $\Psi_{\mathbf{p}}$ up to eight components and introduces a new set of Pauli matrices, $\boldsymbol{l}$, which act on the layer space. The full spin-wave Hamiltonian is $H=\frac{1}{2} \sum_{\mathbf{p}} \Psi_{\mathbf{p}}^{\dagger} \mathbb{M}_{\mathbf{p}} \Psi_{\mathbf{p}}$, with $8 \times 8$ bilinear matrix

$$
\begin{aligned}
\hat{\mathbb{M}}_{\mathbf{p}}= & \frac{1}{2} J_{u d}\left[1+\tau_{1} \zeta_{1} l_{1}\right]+2 J_{0}+\Gamma-\frac{1}{2} \Gamma\left(\gamma_{\mathbf{p}}^{s}+\cos 2 \theta \gamma_{\mathbf{p}}^{d}\right) \zeta_{1} \\
& +\left[2 J_{0} \gamma_{\mathbf{p}}^{s}+\frac{1}{2} \Gamma\left(\gamma_{\mathbf{p}}^{s}+\cos 2 \theta \gamma_{\mathbf{p}}^{d}\right)\right] \tau_{1} \zeta_{1}+D \gamma_{\mathbf{p}}^{p} \tau_{2} \zeta_{2} l_{3} .
\end{aligned}
$$

In addition to the $s$-wave harmonic, we now must introduce the additional cubic harmonics

$$
\begin{aligned}
\gamma_{\mathbf{p}}^{p} & =\sin \theta \sin p_{x}-\cos \theta \sin p_{y}, \\
\gamma_{\mathbf{p}}^{d} & =\frac{1}{2}\left[\cos p_{x}-\cos p_{y}\right],
\end{aligned}
$$

which depend on the Néel order orientation $\theta$ and are of $p$ - and $d$-wave symmetries, respectively. We also have the interlayer exchange constant $J_{u d}>0$ and intralayer exchange energies, which are given in Eq. (5).

This system has four different magnon bands, which can be split into two different representations based on their eigenvalue under the parity operator $\Pi=\zeta_{1} l_{1}$, which commutes with the spin-wave Hamiltonian and assumes the two eigenvalues $\Pi= \pm 1$. Each of these representations is itself doubly degenerate. The dispersions may be obtained analytically, as in Ref. [153], and are given in full in Appendix C. In particular, we find that the even-parity representation $(\Pi=$ +1 ) contains the gapless acoustic pseudo-Goldstone mode, and one gapped optical mode with resonance frequency $\Omega_{0,+-}=\sqrt{\left(2 J_{0}+\Gamma\right)\left(2 \Gamma+2 J_{u d}\right)}$. The odd-parity $(\Pi=-1)$ representation contains two gapped modes with gaps $\Omega_{0,-+}=$ $\sqrt{J_{u d}\left(4 J_{0}+\Gamma\right)}$ and $\Omega_{0,--}=\sqrt{\Gamma\left(4 J_{0}+J_{u d}+\Gamma\right)}$.

Unless $J_{u d}$ is above a critical value (which is not very large for realistic parameters), it will turn out that this mean-field is dynamically unstable since the acoustic mode energies will become complex, signaling the failure of the ansatz of easyplane Néel-order. For $\theta=0$ (Néel order along the $a$ axis), we find a critical threshold of order $J_{u d} / J_{0} \gtrsim 6(\lambda / t)^{2}$, with $\lambda / t \sim .025$ for realistic parameters, which leads to an easily satisfied stability criterion. For the parameters of $t=400 \mathrm{meV}$ and $\lambda=10 \mathrm{meV}$, this is satisfied by $J_{u d} \sim 0.5 \mathrm{meV}$, which we take in the calculations henceforth. The equilibrium spinwave dispersion relation is shown in Fig. 3 and is reasonably well described by a simple easy-plane model with additional interlayer coupling.

Having developed this picture of the equilibrium properties, we now turn our attention toward understanding the role of the magnetoelectric coupling when $g>0$. We will study this in two parts; first we will study the linear coupling of $E^{z}$ to the magnons, which produces magnon-polaritons. We will then examine the quadratic coupling which allows for the squeezing of magnons by the electric field.

\section{MAGNON-POLARITONS}

We now switch on the magnetoelectric coupling $g$. We first note that, except for the presence of the layer-index quantum number in the case of the bilayer model, the coupling 


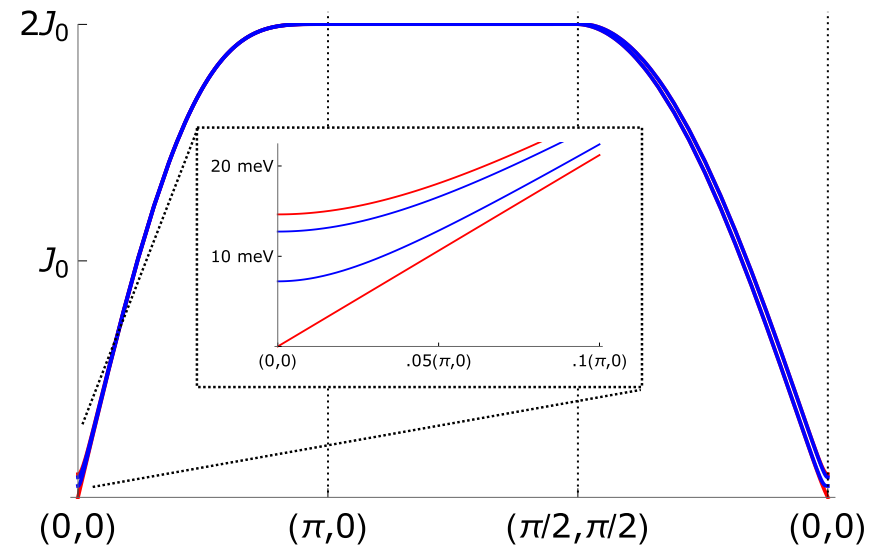

FIG. 3. Spin-wave spectrum of the bilayer system for realistic parameters of $J_{0}=150 \mathrm{meV}, \lambda / t=0.017, J_{u d} / J_{0}=0.0018$ for the case of Néel order along the $a$ axis $(\theta=0)$. We see that is very close to a doubling of the easy-plane dispersion, except for one of the Goldstone modes is rendered massive by the bilayer coupling. Inset: Expanded view of the behavior near the origin, illustrating the masses of various modes. The bands are classified according to their parity under $\Pi=\zeta_{1} l_{1}$, with the upper and lower bands (red) being odd parity and the two middle bands (blue) being even parity.

to in-plane Néel order is the same for the two models. In particular, we will find that there is a linear coupling between the electric field $\hat{E}_{\mathbf{q}}^{z}$ and the spin-wave operators $a_{\mathbf{q}}, b_{-\mathbf{q}}$ which goes as $|\mathbf{q}|$ at long wavelengths. At finite momentum, this will produce hybridization between the magnons and photons at finite momentum-magnon-polaritons. In addition to this linear coupling, we will find a term which couples the electric field $\hat{E}_{\mathbf{q}}^{z}$ to magnon bilinears and tends to a constant as the electric field momentum $\mathbf{q} \rightarrow 0$. We will investigate this coupling in the next section. For now, we start by analyzing the easy-plane toy model.

\section{A. Easy-plane model}

We supplement the easy-plane Hamiltonian Eq. (12) with the magnetoelectric interaction term:

$$
\hat{H}_{\text {int-EP }}=g \sum_{j \in A, \delta} \hat{E}_{j, j+\delta}^{z}\left(\mathbf{e}_{z} \times \boldsymbol{\delta}\right) \cdot \hat{\mathbf{S}}_{j} \times \hat{\mathbf{S}}_{j+\delta} .
$$

We now expand around the ground state found in Sec. III A and the details of this procedure are provided in Appendix B. To first order, we find the magnetoelectric coupling,

$$
\hat{H}_{\mathrm{int}}^{(1)}=\sum_{\mathbf{q}} i \frac{1}{4} g \hat{E}_{-\mathbf{q}}^{z} \gamma_{\mathbf{q}}^{\|}\left(a_{\mathbf{q}}-b_{\mathbf{q}}\right)+\text { H.c. },
$$

with the new longitudinal form factor

$$
\gamma_{\mathbf{q}}^{\|}=2 \cos \theta \sin \frac{q_{x}}{2}+2 \sin \theta \sin \frac{q_{y}}{2} \sim q_{x} \cos \theta+q_{y} \sin \theta
$$

appearing alongside the old $p$-wave form factor. The last relation $\sin q_{j} \sim q_{j}$ is valid due to the long wavelengths of the probe electric field, which is an excellent approximation even in the near field of a terahertz resonator.

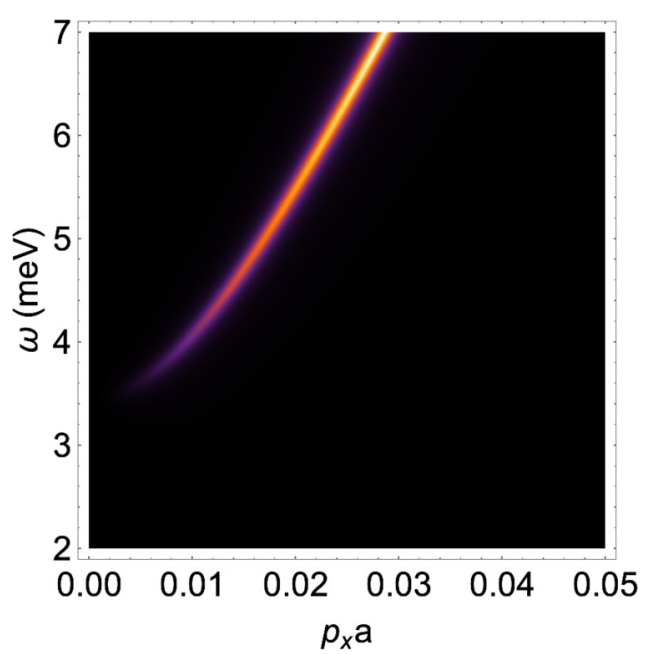

FIG. 4. Magnon contribution to the electromagnetic absorption $\Im \chi_{z z}^{\text {diel }}(\omega, \mathbf{q})$ for the easy-plane model, from Eq. (22). We take characteristic values of $J_{0}=150 \mathrm{meV}, \Gamma=0.01 \mathrm{meV}$, and oscillator strength $F=6.4 \times 10^{4} \mathrm{meV}^{2}$, and choose to consider $\mathbf{q}\|\hat{\mathbf{N}}\| \hat{\mathbf{e}}_{x}$. We note that the effective oscillator strength vanishes as $\mathbf{q} \rightarrow 0$, due to the hybridization form factor $\gamma_{\mathbf{q}}^{\|}$. We also note that the dispersion of the spin wave sets in fairly rapidly.

We now analyze the effect of the linear coupling by determining the linear response of the magnon system to the electric field [203,204]. Standard methods yield the contribution to the $z z$ element of the dielectric susceptibility tensor [225] due to the linear coupling (in lattice units) as

$$
\chi_{z z}^{\text {diel }}(\omega, \mathbf{q})=-\frac{g^{2}\left|\gamma_{\mathbf{q}}^{\|}\right|^{2}}{4} \frac{2 J_{0}\left(1+\gamma_{\mathbf{q}}^{s}\right)}{\left(\omega+i 0^{+}\right)^{2}-\Omega_{\mathbf{q},-}^{2}} .
$$

The imaginary part of this expression is plotted in Fig. 4 in the $\left(\omega, q_{x}\right)$ plane. We can note a few salient features. The first is that the electric field only linearly couples to the massive mode, characterized by $\zeta=-1$, with frequency given by Eq. (15). At long wavelengths, this recovers the spin-wave gap $\Delta_{\mathrm{sw}}=\sqrt{8 J_{0} \Gamma}$.

The next salient feature is that the oscillator strength is clearly proportional to $\mathbf{q}$ through the longitudinal form factor $\gamma_{\mathbf{q}}^{\|}=(\mathbf{N} \cdot \mathbf{q})$, where $\mathbf{N}$ is the direction of the Néel vector. Consequently, this response must arise beyond the dipole approximation and, in general, will require near-field techniques to detect $[141,144,170,172,174,176,226]$. This can be anticipated on symmetry grounds by observing that a fluctuation in the magnetization can only couple to the electric field through a matrix element which is odd under spatial inversion.

In the presence of this coupling, the dielectric function becomes

$$
\epsilon_{z z}^{(1)}(\omega, \mathbf{q}) / \epsilon_{\infty}=1-\frac{F\left|\gamma_{\mathbf{q}}^{\|}\right|^{2}\left(1+\gamma_{\mathbf{q}}^{s}\right)}{\left(\omega+i 0^{+}\right)^{2}-\Omega_{\mathbf{q},-}^{2}} .
$$

The zeros of this indicate the dispersion of the longitudinal collective modes, which are dubbed longitudinal magnonpolaritons. The oscillator strength is given by

$$
F=\frac{g^{2} J_{0}}{\epsilon_{\infty} \mathcal{V}}
$$


Here we have replaced the lattice units, with $\mathcal{V}$ the unit-cell volume and $\epsilon_{\infty}$ is the high-frequency bare dielectric constant. We assume a phonon oscillator strength of $Z_{\mathrm{ph}}^{2} e^{2} / M_{\mathrm{ph}} \epsilon_{\infty} \mathcal{V}=$ $S_{\mathrm{ph}} \sim 2.8 \Omega_{\mathrm{ph}}^{2}$ [220], which yields an estimate of $F \sim 6.2 \times$ $10^{4} \mathrm{meV}^{2}$. However, the actual oscillator strength of the resonance is momentum dependent such that it vanishes at long wavelengths, going as $F a^{2} \mathbf{q}^{2}$ (again, replacing lattice units such that $a$ is the $a b$-plane lattice constant). Therefore, at small momentum the splitting off of the longitudinal magnon-polariton collective mode vanishes and coincides with the standard magnon resonance. To couple a cavity to the magnon-polaritons, one has to ensure that the cavity does not simply couple to the $\mathbf{q}=0$ dielectric response but also samples features from finite momentum. We now address this requirement by considering a toy model of a near-field terahertz resonator.

\section{B. Cavity-magnon polaritons}

The combined requirement of a strong terahertz field and strong terahertz field gradient requires us to couple via a near-field coupling scheme [141,144,170$173,175,176,226-235]$. There are many routes by which this is achievable; we will only explore one potential technique, which essentially combines a terahertz resonator $[75,77,78,86,139,140,148,236]$ with a scanning near-field optical microscopy (SNOM) setup to generate the near-field coupling [141,144,170-173,175,176,226-235]. This particular model is expanded upon in Appendix E. We emphasize that this is only a qualitative discussion of one possible route toward engineering this coupling.

For our purposes, it will be sufficient to model the resonator as an $R L C$ circuit with linewidth $\kappa \sim R / L$ and resonance $\Omega_{\text {cav }} \sim 1 / \sqrt{L C}$, where $L, R, C$ are the effective inductance, resistance, and capacitance, respectively $[77,139,145,148,236,237]$. To generate the near-field coupling, we imagine placing a small metal object, with size of order $R$ within the electric-field antinode of the resonator. For more details, we refer the reader to Appendix E, with a schematic depiction shown in Fig. 13. In the good metal limit, this object will become polarized by the terahertz electric field, with induced polarization

$$
\mathbf{P}_{\text {ind }}=4 \pi \epsilon_{0} R^{3} \mathbf{E}_{\mathrm{cav}} .
$$

Thus, the electric field of the cavity $\mathbf{E}_{\mathrm{cav}}$ induces an effective dipole which oscillates along with the resonator. We then assume that the sample has a thickness $h$ and is placed at a depth $d \sim R$ below the induced dipole. In the presence of the induced dipole, the magnons will contribute to the electrostatic energy of the resonator and therefore change the effective capacitance, such that it includes a contribution from the finite-momentum $\chi_{z z}^{\text {diel }}(\omega, \mathbf{q})$, where the relevant momenta are of order $|\mathbf{q}| \sim 1 / d$.

In Appendix E, we use a simple electrostatic model to evaluate the magnonic substrate contribution to the effective capacitance $K_{\text {magnon }}(\omega)=C(\omega) / C_{\text {bare }}-1$. We find

$$
K_{\text {magnon }}(\omega)=\frac{4 \pi^{2} R^{6} h}{V_{\text {eff }}} \int_{\mathbf{q}} \frac{\chi_{z z}^{\text {diel }}(\omega, \mathbf{q})}{\epsilon_{0}} e^{-2 d|\mathbf{q}|}\left|\mathbf{q} \cdot \mathbf{e}_{\mathrm{cav}}\right|^{2} .
$$

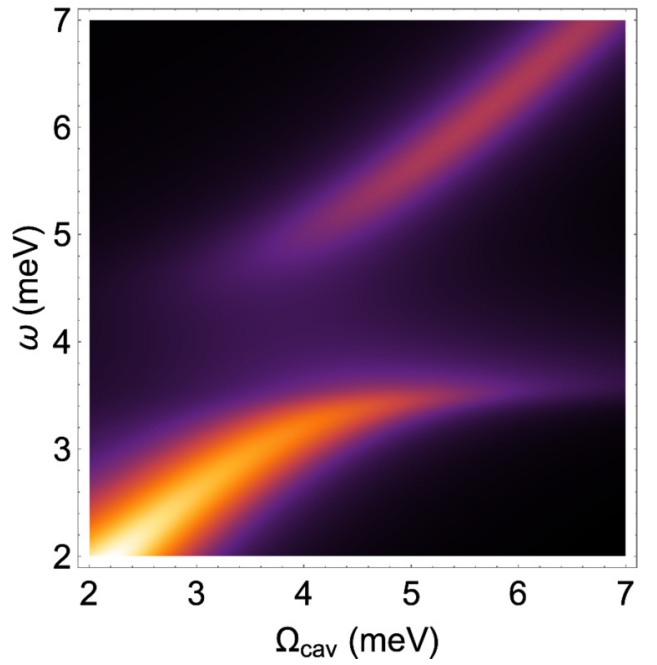

FIG. 5. Cavity spectral function including capacitive coupling to magnons obtained from Eq. (25). We take $V_{\text {eff }}=(40 \mathrm{~nm})^{3}, d \sim R \sim$ $300 a, h \sim 10 a$, and parameters of spin-wave system from main text. We assume a cavity linewidth of $\kappa=1 \mathrm{meV}$. We see a clear avoided crossing in the cavity spectral function as the cavity comes in to resonance with the spin-wave.

Here $\mathbf{e}_{\text {cav }}$ is the unit vector corresponding to the polarization of the electric field at the field antinode, which we take to lie parallel to the $a b$ plane, and $h$ is the sample thickness, which we take in the limit $h \ll d$. For the result in the $h \sim d$ limit, we refer the reader to Appendix E. We also have introduced the effective volume of the cavity, $V_{\text {eff }}$, which is interpreted as the effective volume occupied by the electric field, weighted by the distribution of the electric-field energy density. In the parallel plate model, we would find $V_{\text {eff }}=A_{\text {eff }} l_{\text {eff }}$, where $A_{\text {eff }}$ is the cross-sectional area of the plates and $l_{\text {eff }}$ is their separation.

We evaluate this contribution, taking the oscillator strength estimated in Eq. (23) of $F \sim 6 \times 10^{4} \mathrm{meV}^{2}$, with the resulting cavity spectral function shown in Fig. 5. The conventional notion of strong coupling and ultrastrong coupling do not strictly apply in this sense since the cavity is coupled to a continuum of magnon modes, each with a different resonance and hybridization matrix element. Nevertheless, we may attempt to introduce a suitable notion of hybridization constant, $G_{\text {eff }}$ by studying the ratio of the high-frequency and low-frequency dielectric constants (note that in a single-mode hybridization problem, this is exactly equal to the avoided-crossing size, as per the usual $g^{2} / \Omega_{0}^{2}$ metric. In this case, this turns out to be exactly the value of $K(0)$, so we see that the size of the avoided crossing is governed by

$$
G_{\mathrm{eff}}^{2} / \Omega_{\mathrm{sw}}^{2}=K(0) \sim \frac{h(R / d)^{6} a^{2}}{V_{\mathrm{eff}}} \times F / \Omega_{\mathrm{sw}}^{2} .
$$

In general, this should be evaluated numerically for the chosen cavity geometry, but we argue that one can intuit a scaling relation with the typical momentum $q_{0} \sim 1 / d$, as indicated in the last relation above (here and in this discussion we have replaced explicit lattice units). The scaling of $1 / d^{6}$ is understood as two powers coming from the matrix elementsquared going as $q^{2}$, two powers coming from the onset of the electric field $\left|\mathbf{E}_{\mathbf{q}}\right|^{2} \sim q^{2}$, and the remaining two powers 


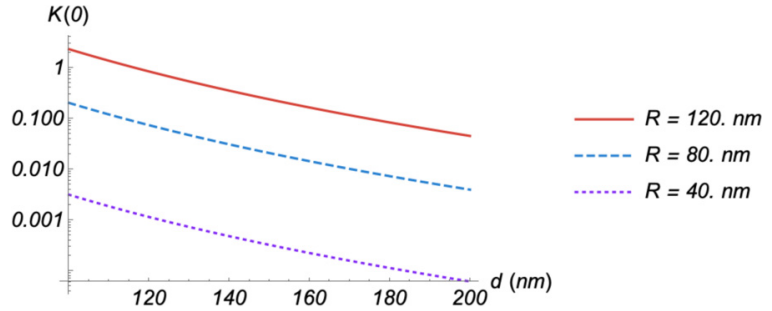

FIG. 6. Size of effective coupling constant, characterized by $K(0)$, as per Eq. (26), as a function of distance $d$ to the resonator dipole, for three different values of the size of the dipole radius $R$ (for details on the specific model, see Appendix E). Other than varying $R$, we use the same parameters as in Fig. 5. In general, this depends on $R$ and $d$ as $(R / d)^{6}$.

coming from the integration measure $d^{2} q$. This is explicitly confirmed numerically, where we plot the evaluated $K(0)$ as a function of the distance parameter $d$ for a variety of values of the polarizability parameter $R$ in Fig. 6 .

Assuming $R \sim d$, this ends up being suppressed by a factor of roughly $a^{2} / A_{\text {eff }}$, where $A_{\text {eff }}$ is the effective cross-sectional area of the capacitor. In what follows, we take an effective mode volume of $V_{\text {eff }} \sim(40 \mathrm{~nm})^{3}$, a sample thickness of $h \sim$ $10 a$, and take $R=d$ for the purposes of our calculations. In this regime, we do find the possibility for $G_{\text {eff }} \sim \Omega_{\mathrm{sw}}$, that is, strong coupling. It is also worth remarking that our toy model is quite crude and a more realistic and optimized model may find even better coupling strengths.

We confirm our dimensional-analysis estimate by numerically evaluating Eq. (25) and computing the resulting dressed cavity spectral function $A_{\text {cav }}(\omega)$ as a function of the bare cavity resonance. In the presence of a finite $K_{\text {magnon }}(\omega)$, we find the cavity spectral function (including Ohmic damping) of

$$
A_{\mathrm{cav}}(\omega)=-\frac{1}{\pi} \mathfrak{s} \frac{1}{\omega^{2}+i \kappa \omega-\Omega_{\mathrm{cav}}^{2} /\left(1+K_{\mathrm{cav}}(\omega)\right)},
$$

as derived in Appendix E. This is illustrated in Fig. 5. We see that as the bare cavity resonance $\Omega_{\text {cav }}$ passes through the bulk spin-wave resonance frequency [238], the dressed cavity spectral function experiences level repulsion, indicating our crude estimate of the strong-coupling regime was correct.

\section{Bilayer-Rashba model}

The bilayer Rashba Hamiltonian is only slightly more complicated to analyze than the easy-plane Hamiltonian. The bulk of the calculation is carried out in Appendix $\mathrm{C}$ and we merely present the result here. By expanding the interaction Hamiltonian in Eq. (11) up to quadratic order, we obtain essentially the same linear interaction but now summed over the two layers

$$
H_{\mathrm{int}}^{(1)}=\frac{i g}{4} \sum_{\mathbf{p} \ell} \hat{E}_{-\mathbf{p}}^{z} \gamma_{\mathbf{p}}^{\|}\left(a_{\mathbf{q} \ell}-b_{\mathbf{q} \ell}\right)+\text { H.c. }
$$

We apply the same methods as in the easy-plane case, however, we must now also keep track of the layer quantum number. We see immediately that the electric field only couples to the $\zeta_{1}=-1$ and $l_{1}=+1$ modes. The complication is that, in the presence of the DM interaction, $l_{1}$ is no longer a good quantum number. Therefore, we evaluate in the energy eigenbasis and then take the appropriate matrix element, producing the dielectric susceptibility

$$
\chi_{z z}^{\operatorname{diel}}(\omega, \mathbf{q})=-\frac{g^{2}\left|\gamma_{\mathbf{q}}^{\|}\right|^{2}}{16} w^{T} \cdot \hat{\mathbb{D}}^{R}(\omega, \mathbf{p}) \cdot w,
$$

where $w^{T}=(1,1,-1,-1,1,1,-1,-1)$ is the (nonnormalized) Nambu spinor corresponding to the direct product of the +1 eigenstates of $\tau_{1}$ and $l_{1}$, and -1 eigenstate of $\zeta_{1}$, and $\mathbb{D}^{R}=\left(\left(\omega+i 0^{+}\right) \tau_{3}-\hat{\mathbb{M}}_{\mathbf{p}}\right)^{-1}$ is the retarded spin-wave propagator for the bilayer system, with $\hat{\mathbb{M}}_{\mathbf{p}}$ the quadratic form from Eq. (16).

The form of the dissipative part of the dielectric susceptibility in Eq. (29) is depicted in Fig. 7 for a few different orientations of the in-plane Neel vector relative to the $a$ axis, while scanning momentum $\mathbf{q}$ also along the $a$ axis. We note this has a slightly more complicated behavior due to the absence of spin-rotation symmetry, but broadly speaking it is qualitatively similar to the easy-plane system. What is not visible in the spectral plots of Fig. 7 is that another higher-lying magnon branch also is optically active at finite momentum, but has a much smaller oscillator strength and we will not dwell on this mode.

We again analyze the near-field interaction with a terahertz cavity using the setup outlined in the previous subsection. The derivation of Eq. (25) remains valid if we use the correct dielectric susceptibility $\chi_{z z}^{\text {diel }}$, which was just computed above in Eq. (29). Likewise, the cavity spectral function retains the same dependence on $K_{\text {magnon }}$. This is depicted for a particular orientation of Neel order in Fig. 8.

As we can see, the result is qualitatively similar to the case of the single monolayer. To summarize, we clearly see that at finite momentum, spin-orbit coupling admixes spin waves and optical phonons such that magnons acquire a finite electric dipole moment. This then allows for their coupling to cavity photons, provided there is a sufficiently large electric field gradient. In the section above, we outline one possible way to generate the necessary field gradient-however, we emphasize that the scheme proposed above is only one of many potential ways this may be done. Other promising routes may involve making smaller split-ring resonators with larger fringing fields, using wave-guide based modes [83,143], plasmonic nanocavities [227], or employing photonic crystal metamaterials [177].

In contrast, in the next section we will consider a different coupling mechanism which allows for the cavity to strongly couple to pairs of magnons without the need to introduce nearfield coupling schemes.

\section{BIMAGNON INTERACTION}

In this section, we will demonstrate how, in a bilayer system like YBCO, the same optical phonon can also lead to a coupling between the cavity electromagnetic field and pairs of magnons-bimagnons. Furthermore, this mechanism doesn't rely on spin-orbit coupling and therefore is anticipated to be much stronger. In principle, this mechanism can be used to generate correlated pairs of magnons [16,23,58,59,195,239] via parametric driving from the cavity field, but in this paper 

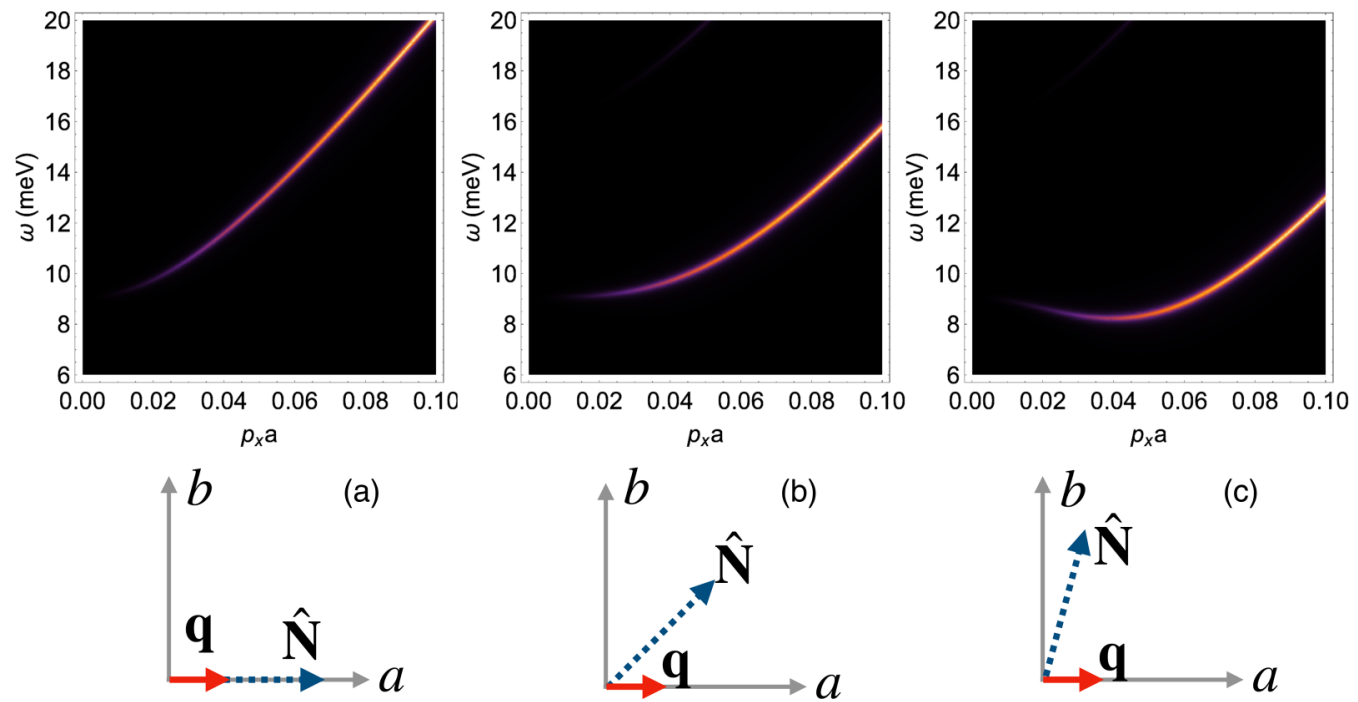

FIG. 7. Magnon contribution to the $\Im \chi_{z z}^{\text {diel }}(\omega, \mathbf{q})$ for the Rashba bilayer model, from Eq. (29). We again observe the vanishing oscillator strength at long wavelengths. In this case, the dispersion relation of the magnon is less trivial and we illustrate the susceptibility for three different configurations of the in-plane momentum and Neel order, illustrated in the lower part of the figure. This is shown specifically for three scenarios of Neel order at angles relative to the $a$ axis of (a) $\theta=0$, (b) $\theta=45^{\circ}$, and (c) $\theta=75^{\circ}$, respectively. We for simplicity only show q $\| a$. While it is not visible on the chosen scale, there is a small contribution for a higher-lying spin-wave band which behaves similarly as the contribution from the dominant band.

we will focus on the linear response regime and leave a more in-depth analysis to future work.

\section{A. Cavity-bimagnon interaction}

For brevity, we will focus on the linear response of the bilayer system to a homogeneous electric field and limit our attention to the larger $g^{\prime}$ coupling. As a reminder, this coupling is due to the buckled nature of the bilayer structure in equilibrium, and is independent of spin-orbit coupling. In the buckled

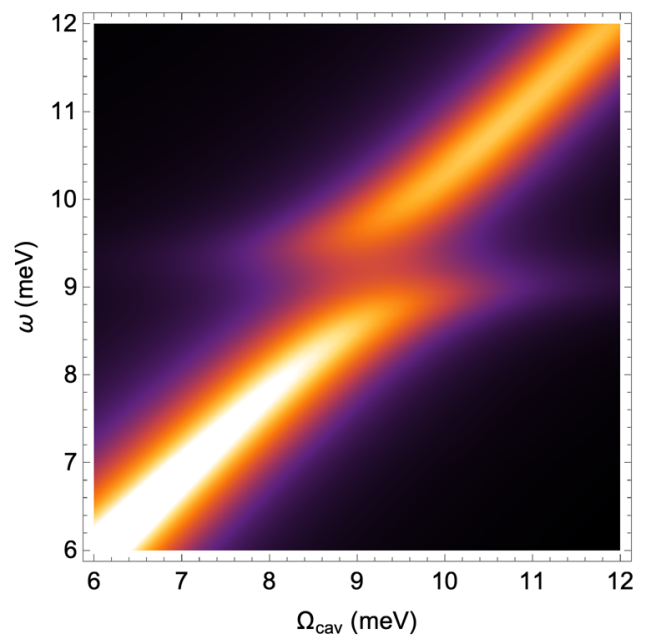

FIG. 8. Cavity spectral function including capacitive coupling to magnons obtained from Eq. (25) and using $\chi_{z z}^{\text {diel }}$ as obtained in Eq. (29). We take $V_{\text {eff }}=(40 \mathrm{~nm})^{3}, d \sim R \sim 300 a, h \sim 10 a$, and parameters of spin-wave system from main text. We assume a cavity linewidth of $\kappa=1 \mathrm{meV}$. We see a clear avoided crossing in the cavity spectral function as the cavity comes in to resonance with the spin wave. structure, the $\mathrm{Cu}-\mathrm{O}-\mathrm{Cu}$ bond length and angle vary linearly with phonon displacement, and oppositely between the two layers (such that overall inversion symmetry is preserved).

The relevant term in the Hamiltonian is

$$
H_{\text {int }}=\sum_{j \in A \delta \ell} g^{\prime}(-1)^{\ell} \hat{E}^{z} \mathbf{S}_{j \ell} \cdot \mathbf{S}_{j+\delta \ell},
$$

where $g^{\prime}=Z_{\mathrm{ph}} e / M_{\mathrm{ph}} \Omega_{\mathrm{ph}} \times 8 t \alpha / U$, and $\alpha=\left.\frac{d t}{d Q}\right|_{\mathrm{eq}}$ is the equilibrium linear variation of the nearest-neighbor hopping with respect to the oxygen displacement. We see the $(-1)^{\ell}$ factor ensures the coupling has odd parity under inversion, which swaps the two layers and also inverts the electric field z-component $\hat{E}_{z}$.

Applying the linear spin-wave expansion, we find that the lowest order coupling due to Hamiltonian Eq. (30) is quadratic in the Holstein-Primakoff bosons. We obtain the coupling (at zero electric field momentum)

$$
\hat{H}_{\mathrm{int}}^{(2)}=\frac{1}{2} \sum_{\mathbf{p}} \Psi_{\mathbf{p}}^{\dagger} \hat{E}^{z} \hat{\mathbb{V}}_{\mathbf{p}} \Psi_{\mathbf{p}}
$$

with the scattering vertex

$$
\hat{\mathbb{V}}_{\mathbf{p}}=2 g^{\prime} l_{3}\left[1+\gamma_{\mathbf{p}}^{s} \tau_{1} \zeta_{1}\right]
$$

Here $\Psi_{\mathbf{p}}$ is the eight-component Nambu spinor from Sec. III.

To simplify the calculations, we will replace the full microscopic Rashba bilayer model with an approximately equivalent model of two layers with in-plane easy-plane anisotropy and weak interlayer coupling, such that the relevant quadratic form for spin-waves is $\hat{\mathbb{M}}_{\mathbf{p}}=\frac{1}{2} J_{u d}\left[1+\tau_{1} \zeta_{1} l_{1}\right]+$ $2 J_{0}-\Gamma \gamma_{\mathbf{p}}^{s} \zeta_{1}+\left(2 J_{0}-\Gamma\right) \gamma_{\mathbf{p}}^{s} \zeta_{1} \tau_{1}$. This dispersion is depicted in Fig. 9(a), and in more detail near the $\Gamma$ point in Fig. 9(c). 
(a)

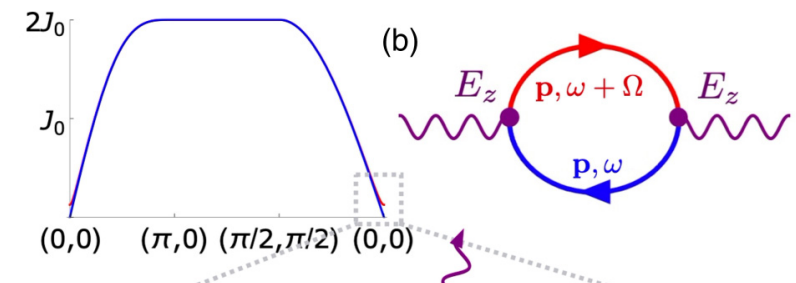

(c)

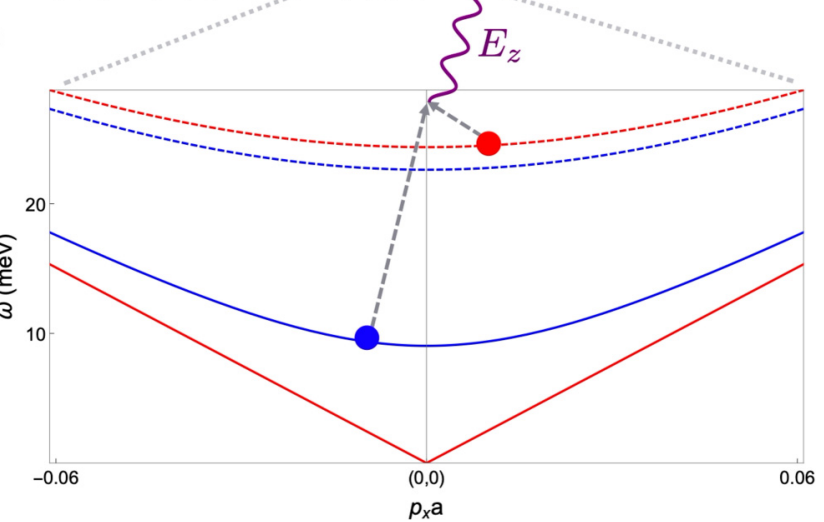

FIG. 9. Magnon bands in the bilayer system: (a) The set of four magnon bands for $J_{0}=128 \mathrm{meV}, J_{u d}=0.5 \mathrm{meV}$, and $\Gamma=0.016$ $\mathrm{meV}$; (b) Feynman diagram illustrating the bimagnon contribution to the dielectric susceptibility; (c) expanded view of the magnon band dispersion near the center of the Brillouin zone, with the interband pairs of magnons with opposite momenta shown schematically.

We find, in general, four bands with dispersions

$\Omega_{\mathbf{p}, l}^{(\zeta)}=\sqrt{\left(2 J_{0}-\Gamma \gamma_{\mathbf{p}}^{s} \zeta+\frac{1}{2} J_{u d}\right)^{2}-\left(\left[2 J_{0}-\Gamma\right] \gamma_{\mathbf{p}}^{s}+\frac{1}{2} J_{u d} l\right)^{2}}$,

where $\zeta= \pm$ is the parity under sublattice symmetry $\zeta_{1}$, and $l= \pm 1$ is the parity under bilayer inversion $l_{1}$.

To compute the response, we use the Matsubara finitetemperature method to compute the magnon contribution to the electromagnetic response function, corresponding to the Feynamn diagram in Fig. 9(b). We present the result at $T=0$, and after performing the relevant analytic continuations.

Since the scattering vertex in Eq. (32) commutes with the sublattice parity $\zeta_{1}$, we can express the resulting response function $\chi_{z z}^{\text {diel }}$ as a sum over two processes-one for each value of $\zeta$. Furthermore, because the vertex flips the interlayer parity $l_{1}$, we find that only interband processes contribute to $\chi^{\text {diel }}$, as depicted in Fig. 9(c). We therefore find

$$
\chi_{z z}^{\mathrm{diel}}(\omega)=\chi_{z z}^{(+)}(\omega)+\chi_{z z}^{(-)}(\omega),
$$

indicating the contribution from the bands with $\zeta=+1,-1$ respectively. The full result of this calculation is cumbersome and relegated to Appendix C 7 a. In the following, we will numerically evaluate the resulting expressions, as well as present simplified results in the appropriate regimes.

We begin by studying the limiting case when anisotropy $\Gamma=0$. At this point, the result simplifies further since both of the contributions $\chi^{( \pm)}$become degenerate. This leads to the

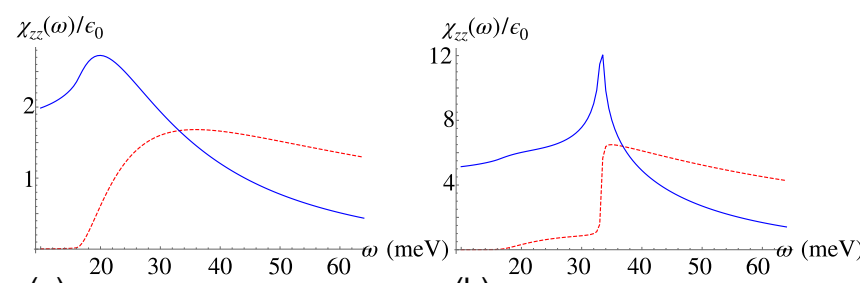

(a)

FIG. 10. Contribution to the dielectric susceptibility due to bimagnon coupling, corresponding to the Feynman diagram in the inset of Fig. 9(b). We show the real part (solid blue) and imaginary part (dashed red) of the relative susceptibility $\chi_{z z}^{\text {diel }}(\omega, \mathbf{q}=0) / \epsilon_{0}$ as a function of the electric field frequency $\omega$. We use scalar exchange $J_{0}=128 \mathrm{meV}$ and take interlayer exchange $J_{u d}=0.5 \mathrm{meV}$, compatible with our estimates from Sec. III B. (a) Response when easy-plane anisotropy $=0$, corresponding to the case of two doubly degenerate magnon bands. The response sets in once the single-magnon threshold is passed, since in this case the bimagnon is actually a single gapped magnon, along with a soft Goldstone mode. (b) Response when $\Gamma=0.16 \mathrm{meV}$, the value obtained in previous sections. The dominant feature corresponds to a bimagnon excitation gap $\Omega_{\text {bimag }} \sim$ $33 \mathrm{meV}$. In contrast to (a), we find a strong response at this frequency driven by the Van Hove singularity, which in two dimensions is logarithmic for the real part and steplike for the imaginary part.

analytic form

$\chi_{z z}^{\text {diel }}(\omega)=\int_{\mathbf{p}} \frac{8\left(g^{\prime}\right)^{2}\left[1-\left(\gamma_{\mathbf{p}}^{s}\right)^{2}\right]\left(\Omega_{-, \mathbf{p}}-\Omega_{+, \mathbf{p}}\right)^{2}\left(\Omega_{-, \mathbf{p}}+\Omega_{+, \mathbf{p}}\right)}{\Omega_{-, \mathbf{p}} \Omega_{+, \mathbf{p}}\left[\left(\Omega_{-, \mathbf{p}}+\Omega_{+, \mathbf{p}}\right)^{2}-\left(\omega+i 0^{+}\right)^{2}\right]}$.

Here we have suppressed the dependence on the $\zeta$ quantum number, since the $\zeta= \pm 1$ bands are both degenerate in this limit.

There is a nontrivial response even when $\Gamma=0$, with the corresponding dielectric susceptibility $\chi_{z z}^{\text {diel }}$ shown in Fig. 10(a). We see that the response is fairly muted and mostly corresponds to an onset in the electromagnetic absorption once the threshold for magnon pair creation is surpassed. Note that because when $\Gamma=0$, one magnon branch involved in the pair processes is gapless, such that the two-magnon threshold energy coincides with the single-magnon threshold energy of the gapped band.

In contrast, when $\Gamma>0$ we find the response exhibits a singular response due to the Van Hove singularity in twodimensions. This is illustrated in Fig. 10(b). We see that there is a meager response as the frequency passes through the single-magnon threshold, but this is largely overshadowed by the response at the two-magnon threshold corresponding to the production of two gapped magnons depicted in Fig. 9(c) and also clearly visible in the dielectric response shown in Fig. 10(b).

To understand the origin of this strong bimagnon response, we study the contribution $\chi_{z z}^{(-)}(\omega)$, which originates from the production of two magnons, one on each of the $\zeta=-1$ bands. We find this assumes the form

$$
\chi_{z z}^{(-)}(\omega)=\int_{\mathbf{p}} \frac{C_{\mathbf{p}}^{(-)}}{\left(\Omega_{\mathrm{p}-}^{(-)}+\Omega_{\mathrm{p}+}^{(-)}\right)^{2}-\left(\omega+i 0^{+}\right)^{2}},
$$




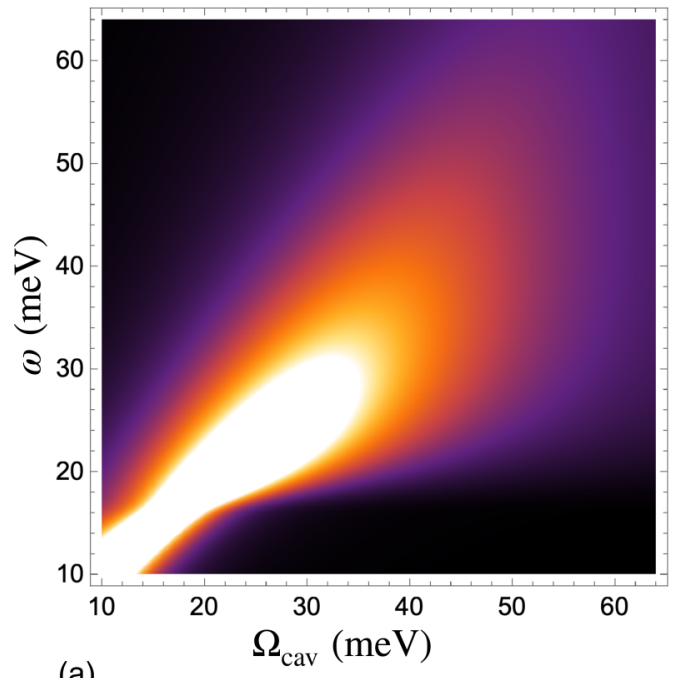

(a)

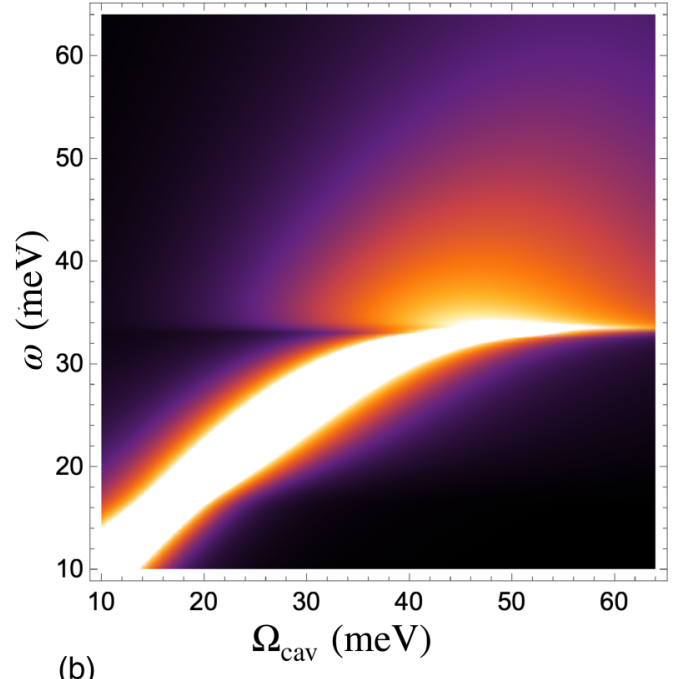

(b)

FIG. 11. Cavity spectral functions include contribution to the dielectric susceptibility due to bimagnon coupling illustrated in Fig. 7. (a) Density plot illustrating the behavior of the cavity spectral function $A_{\text {cav }}(\omega)$ as the bare cavity resonance frequency $\Omega_{\text {cav }}$ is tuned across the bimagnon resonance for the case of $\Gamma=0$, which corresponds to $\chi_{z z}$ from Fig. 7(a). Upon crossing the threshold for pair production, the imaginary part of $\chi^{\text {diel }}$ sets in rapidly, leading to a mostly dissipative response and resulting diffuse line shape. (b) Density plot for cavity spectral function with finite $\Gamma=0.16 \mathrm{meV}$. At the edge of the bimagnon continuum, located around $\omega=33 \mathrm{meV}$, the logarithmic singularity manifests as a visible feature in the cavity spectral function. Though not a true avoided crossing due to the onset of strong damping by the continuum, we do see some type of level repulsion as we attempt to tune the bare cavity frequency $\Omega_{\text {cav }}$ through the resonance. Note the cavity spectral function is renormalized such that the resonance occurs at $\omega^{2}=\Omega_{\text {cav }}^{2}$ as $\Omega_{\text {cav }}^{2} \rightarrow 0$.

where we recall that $\Omega_{\mathbf{p}, l}^{(\zeta)}$ are given in Eq. (33) and $C_{\mathbf{p}}$ is a complicated expression involving matrix elements, which importantly tends to a constant as $\mathbf{p} \rightarrow 0$. Therefore, at low frequencies we may expand the integrand for small momentum $\mathbf{p}$ and invoke rotational symmetry of the resulting expansion, such that the dispersion may be characterized by the bimagnon dispersion

$$
\Omega_{\mathrm{p}-}^{(-)}+\Omega_{\mathrm{p}+}^{(-)} \sim \Omega_{\text {bimag }}+\frac{1}{2 M_{\text {bimag }}} \mathbf{p}^{2}+O\left(|\mathbf{p}|^{3}\right),
$$

where the bimagnon resonance occurs at

$$
\Omega_{\text {bimag }}=\Omega_{-, 0}^{(-)}+\Omega_{+, 0}^{(-)}=\sqrt{8 \Gamma J_{0}}+\sqrt{8 J_{0}\left(\Gamma+J_{\mathrm{ud}} / 2\right)} .
$$

We now see that the resulting integral acquires a singular contribution due to the quadratic nature of the dispersion of the bimagnon state, which leads to a logarithmically divergent contribution to the real-part of the response function $\chi_{z z}^{(-)}$in two dimensions.

In contrast, this is not the case for the other contribution since, as previously established, the bimagnon excitation involves one gapped spin wave and one soft Goldstone mode, which therefore yields a linear dispersion at sufficiently small momenta. We will, however, note in passing that, in principle, due to the lower orthorhombic symmetry of real cuprate materials, there are further magnetocrystalline anisotropies which will ultimately gap out this Goldstone mode, perhaps leading to another singular contribution. We leave the study of these more realistic models to future work [240].

Finally, given that we find a strong bimagnon contribution to the dielectric susceptibility, we consider a simple model of coupling to a cavity $[77,139,145,148,236,237]$ to assess whether we may find resulting bimagnon-polaritons. Using the computed dielectric response functions $\chi_{z z}^{\text {diel }}(\omega)$, we determine the effective capacitance of a hypothetical cavity enclosing the cuprate sample, with the homogeneous field polarized along the $c$ axis, such that $C(\omega)=A_{\mathrm{eff}} / \ell_{\mathrm{eff}} \epsilon_{z z}(\omega)$. We then study the cavity spectral function:

$$
A_{\mathrm{cav}}(\omega)=-\frac{1}{\pi} \mathfrak{s} \frac{1}{\omega^{2}+i \gamma \omega-\Omega_{\mathrm{cav}}^{2} \epsilon_{z z}(0) / \epsilon_{z z}(\omega)} .
$$

The factor of $\epsilon_{z z}(0) / \epsilon_{z z}(\omega)$ arises because we have specifically chosen to parametrize $\Omega_{\text {cav }}$ such that the dressed resonance agrees with the bare cavity frequency $\Omega_{\text {cav }}$ as $\Omega_{\text {cav }} \rightarrow 0$. The resulting cavity spectral function is shown in Fig. 11(a) for the case of $\Gamma=0$, and Fig. 11(b) for $\Gamma>0$ (parameter choice in caption).

We see that neither case exhibits a conventional strongcoupling splitting near the bimagnon feature, due to it being more like a two-particle continuum than true resonance. In the case of $\Gamma=0$, we see that once the cavity resonance crosses the peak in $\mathfrak{R} \epsilon(\omega)$, it rapidly dissolves into a diffuse line shape. While this does not mean that the coupling is weak (in fact, it implies the coupling is strong enough to substantially damp the cavity), it does mean that the resonance is too broad to participate in coherent level repulsion and is largely dissipative in nature.

For finite $\Gamma>0$, shown in Fig. 11(b), we see a more complicated line shape emerge due to the singular nature of the bimagnon coupling. We investigate this further in Fig. 12 by examining line cuts of the spectral function at constant cavity resonance frequency $\Omega_{\text {cav }}$. We see the strong coupling leads to an asymmetric split line shape which shows some signatures of a coherent avoided crossing. Increasing the cavity resonance further, we find the coherent contribution is quickly 


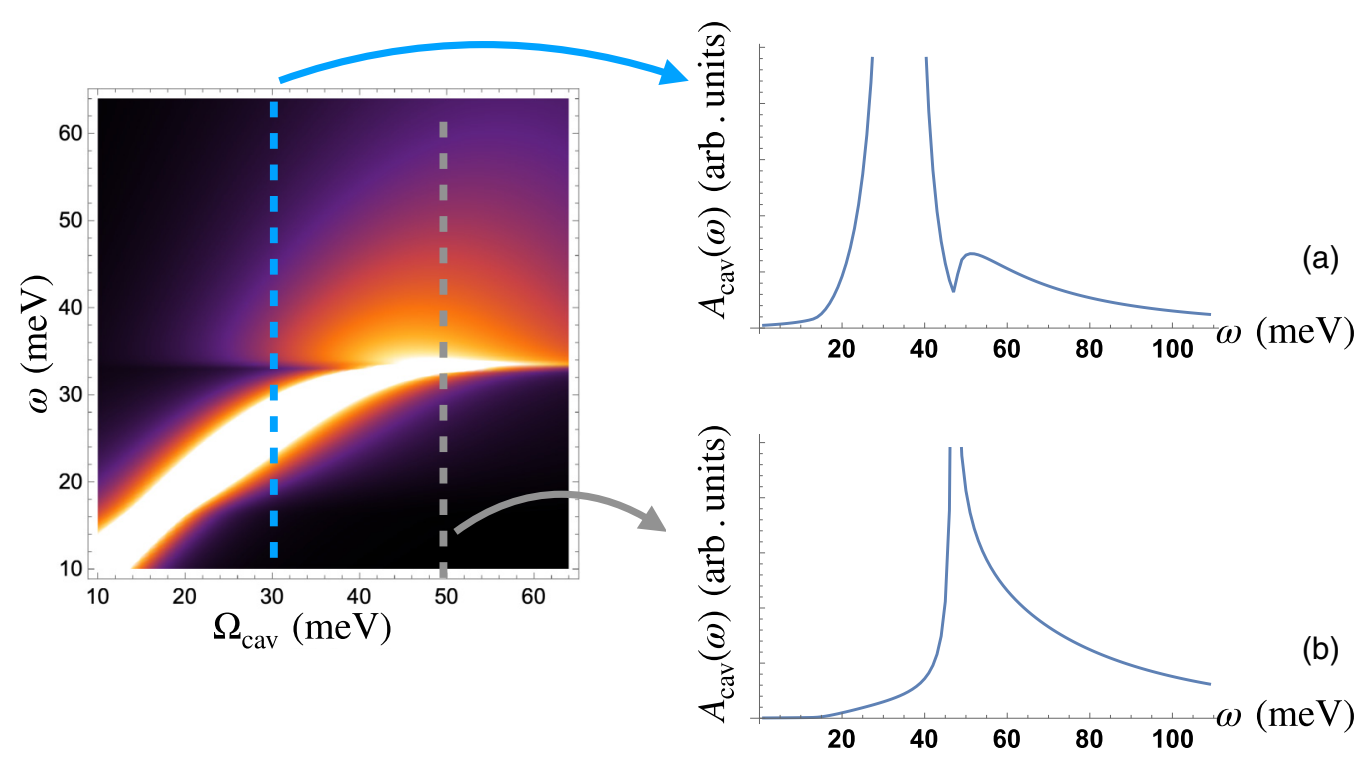

FIG. 12. Line cuts of cavity spectral function from Fig. 11(b), with finite $\Gamma=0.16 \mathrm{meV}$ for constant $\Omega_{\text {cav }}$. (a) Line cut along $\Omega_{\text {cav }}=$ $30 \mathrm{meV}$. We see a small secondary shoulderlike feature appears in the cavity spectral function at the bimagnon resonance, but it is largely overshadowed by the bare resonance of the cavity. (b) As the cavity is tuned through the resonance, such that now $\Omega_{\text {cav }}=50$ meV, the shoulderlike feature becomes washed out be a broad overdamped continuum. In this case, the dominant effect of the magnons is to provide an asymmetric damping to the cavity line shape, which essentially just reflects this contribution.

overcome by the strong damping, which now dominates the cavity spectral function. Therefore, it is plausible that in the presence of the strong bimagnon coupling for finite $\Gamma>0$, strong coherent cavity-magnon interactions may also be observed, provided the cavity lies below the continuum, which rapidly destroys any notion of avoided crossing.

\section{B. Implications for pairing and paramagnons}

Before concluding, we briefly discuss the implications of our work on magnon-mediated pairing in cuprates. We assume the magnons form the bosonic glue which causes electrons to form Cooper pairs. As extensively studied for the case of phonon-mediated pairing in, e.g., Ref. [182], the parametric excitation of the bosonic degree of freedom can enhance its ability to induce electron pairing. This results from the dynamical softening of the bosonic mode, leading to significant enhancement of the retarded response of these bosons in the vicinity of the parametric resonance. However, as we discuss below, extrapolation of these ideas to magnon-induced pairing is not straightforward. We start our discussion by considering the overdoped regime. In this case, the Neel order parameter is completely destroyed and the excitations of the electron liquid in the spin channel are represented by the so-called paramagnons. Coherence of these excitations depends on the geometry of the Fermi surface as discussed in Ref. [241]. In particular, if the decay of paramagnons into electron-hole excitations is kinematically possible, their dynamics is strongly damped. In the spin-density wave picture, the paramagnon propagator can be straightforwardly obtained within the random phase approximation and has the following form [241]:

$$
\chi_{\mathrm{SDW}}^{-1}\left(i \omega_{n}\right) \propto \omega_{n}^{2}+\gamma\left|\omega_{n}\right|+\Delta_{\mathrm{SDW}}^{2},
$$

where $\gamma$ is a constant which depends on the Fermi surface geometry and $\Delta_{\mathrm{SDW}}$ is the spin-wave gap. In this regime, parametric resonance [182] is difficult to achieve due to the strong dissipation [242].

Alternatively, in the underdoped regime when the Neel order is not completely destroyed by electron excitations, the magnon structure remains approximately the same as at the exact half filling, as described in Appendix B. Propagation of a single hole (holon) dressed by the magnon environment is studied in, e.g., the Kane-Lee-Read manner [104]. One may then consider applying our scheme to allow for the parametric excitation of magnons as studied in Sec. V, now also including the coupling to the magnetic polaron's motion, near the parametric resoance in a similar manner as was studied in the phonon-mediated case [182]. The effect on electron pairing may then be revealed by studying propagation of two holons subject to the parametric coupling. However, this is a hard problem and lies beyond the scope of the current paper, and will be studied in a future publication.

\section{CONCLUSION}

In conclusion, we have examined a variety of ways in which a terahertz cavity can be coupled to the spin fluctuations in a Mott insulating antiferromagnet, which is meant to model the parent compound for a high- $T_{c}$ cuprate superconductor. In our paper, we examined how an infrared-active phonon mode (in this case, associated to the in-phase motion of the planar oxygens) can mediate the coupling between the spin waves and the cavity [126]. The first scheme, outlined in Sec. IV, takes spin-orbit coupling into account [23,160-168] and is in principle present in both monolayer and bilayer cuprate systems. In a second scheme, we considered a 
coupling to the scalar exchange interaction due to linear changes of the ligand bond angle; this can only happen in a bilayer systems when inversion symmetry is broken [114,178]. We then proceeded to show how this phonon-mediated spinorbit mechanism could induce hybridization of the cavity photons with the Neel magnons-forming Neel magnonpolaritons.

Though always present, these Neel-magnon-polaritons only hybridize at finite momenta and therefore require nearfield terahertz engineering to be detected. While it presents an engineering challenge, the technologies associated to near-field coupling of terahertz resonators has seen rapid development and is now quite promising [26,75,77,78,139141,144,148,170-173,175,176,226-231,233-235].

We then studied the coupling to the bimagnons through phonon-modulated superexchange, which is only present in the bilayer system. In this case, it was found that the cavity only couples to the magnon bilinear operator in the absence of spin-orbit interactions. We nevertheless show that this bimagnon coupling still leads to a significant dielectric response, at a frequency governed by the sum of the two relevant magnon band gaps. In all likelihood, when present, this response will dominate over the spin-orbit mediated mechanism and allow for the strong coupling of cavity photons to the bimagnon resonance. Furthermore, going beyond linear response, it is evident that the bimagnon coupling outlined can also be used as a parametric drive, which presents very interesting possibilities for future studies [16,23,58,59,195,239].

Coupling of cavity photons to antiferromagnetic spin fluctuations, as we have proposed in this paper, opens the door to the study of a number of very interesting possibilities. One such avenue is to study whether strong coupling to a cavity can be used to enhance (or destroy) existing antiferromagnetic order or even produce novel magnetically ordered phases $[9,10,14]$. This would require extending our work to include effects such as strong frustration or spin-orbit coupling, both of which may lead to novel magnetic interactions.

Another extension, which is even more directly related to our paper, is to study the effect of finite carrier (e.g., hole) doping [101]. At finite doping, charge carriers are known to strongly interact with the background antiferromagnetic order, as well as the phonon modes themselves [56,178], and may exhibit a number of competing tendencies [91,112,243], including antiferromagnetic order, superconductivity, charge order [90,244], and possibly even topological order $[111,116,117,245]$. The possibility of manipulating this complex interplay using strong coupling to cavity photons may open a new avenue toward control of strongly correlated electronic materials [79].

As a preliminary study, determining the dynamics of a single hole moving in the background of the antiferromagnetic order, in the presence of the strong cavity dressing, already presents a compelling, but daunting, theoretical effort $[87,88,104,111,113,115,117,178,246]$. It would also be interesting to approach the problem from the Fermi-liquid perspective, treating the antiferromagnetic order as a collective mode of the Fermi liquid [103,109,110]. In this case, the coupling between the plasma oscillations of the electron Fermi liquid, cavity, and antiferromagnetic collective modes are all taken to be important. This may be important in the context of overdoped cuprates [91,110,112,116], nickelates [247-251], correlated oxide heterostructures [166,252,253], and other compounds with spin-density wave tendencies [107,116,254].

More broadly, the mechanism we discuss can easily be generalized to other kinds of magnetic systems. Perhaps some of the more interesting candidates would be various realizations of spin liquids in strongly correlated materials [9,14,53,192,209,255,256], iridate compounds with large spin-orbit interactions [127,257-259], electron-doped cuprates [260-264], or two-dimensional van der Waals materials $[26,126,143,149,174,177,189,265,266]$. Generically, we find that the cavity coupling is qualitatively enhanced by large spin-orbit interactions, and also in the presence of bilayer unit cells, which naturally lead to the above candidates.

Finally, we comment that our work is also relevant within the context of cavity sensing techniques [84] and may be useful for detecting novel properties of quantum matter [65,267-270]. This is particularly true once the system is doped away from the insulating phase, and especially within the enigmatic pseudogap region, where strong coupling to an electromagnetic resonator may afford further insight into puzzling reports of time-reversal symmetry breaking [268,271,272] and inversion symmetry breaking [169,273,274].

\section{ACKNOWLEDGMENTS}

The authors thank Dominik Juraschek, Fabian Menges, Ilya Esterlis, Stephan Jesse, Jérôme Faist, Andrea Cavalleri, Dmitri N. Basov, Ataç İmamoğlu, Richard Averitt, and Amir Yacoby for fruitful discussions regarding the paper. Work by J.B.C., N.R.P., and P.N. was partially supported by the Quantum Science Center (QSC), a National Quantum Information Science Research Center of the U.S. Department of Energy (DOE). J.B.C. gratefully acknowledges support from the Harvard Quantum Initiative. N.R.P. is supported by the Army Research Office through an NDSEG fellowship. V.G. and A. G. were supported by NSF No. DMR-2037158, US-ARO Contract No. W911NF1310172, and Simons Foundation. P.N. gratefully acknowledges support through Grant No. GBMF8048 from the Gordon and Betty Moore Foundation. E.D. and J.B.C. were supported by Harvard-MIT CUA, AFOSR-MURI: Photonic Quantum Matter Award No. FA95501610323, the ARO grant Control of Many-Body States Using Strong Coherent Light-Matter Coupling in Terahertz Cavities, and the Harvard Quantum Initiative.

\section{APPENDIX A: LOW-ENERGY THEORY OF HUBBARD MODEL}

Here we derive the low-energy superexchange interactions by integrating out charge fluctuations in the half-filled Hubbard model. We use a single-band Hubbard model with both regular and spin-orbit hopping of the form

$$
H=-\sum_{j} \sum_{\delta} c_{j+\delta \alpha}^{\dagger} t_{\delta}^{\alpha \beta} c_{j \beta}+U \sum_{j} n_{j \uparrow} n_{j \downarrow},
$$

where $\delta$ label the nearest-neighbor lattice sites of site $j$ and $\alpha, \beta$ are the spin-quantum numbers of the electrons. We assume that the Hubbard $U \gg t$ so we are well within the 
localized regime. The hopping matrix elements $t_{\delta}^{\alpha \beta}$ represent the amplitude for an electron of spin $\beta$ to hop to the neighboring site in the $\delta$ direction, with a final spin state of $\beta$. Time-reversal symmetry implies that we can write these as

$$
t_{\delta}^{\alpha \beta}=t_{\delta} \delta_{\alpha \beta}+i \lambda_{\delta} \cdot \sigma_{\alpha \beta}
$$

with $t, \lambda$ real parameters. Hermiticity and translational symmetry constrain these to obey $t_{\delta}=t_{-\delta}$ and $\lambda_{\delta}=-\lambda_{-\delta}$.

We now implement the Schrieffer-Wolf elimination of the doublon states. Let $\mathcal{P}_{n}$ be the projector onto the subspace with $n$ total doublons. We will restrict to the limit where only $\mathcal{P}_{0}, \mathcal{P}_{1}$ are needed, which is sufficient for second-order perturbation theory. We can partition the Hamiltonian into an effective block structure as

$$
H=\left(\begin{array}{cc}
0 & \mathcal{P}_{0} H \mathcal{P}_{1} \\
\mathcal{P}_{1} H \mathcal{P}_{0} & U
\end{array}\right),
$$

where we have used the fact that at exactly half filling $\mathcal{P}_{0} H \mathcal{P}_{0}=0$ and $\mathcal{P}_{1} H \mathcal{P}_{1}=U \mathcal{P}_{1}$ to lowest order in $t / U$. We then can adiabatically eliminate the doublon subspace, valid at low-frequencies $\omega \ll U$, such that we end up with an effective Hamiltonian (which acts on the half-filled manifold):

$$
H_{\mathrm{eff}}=-\frac{1}{U} \sum_{j} \sum_{\delta} c_{j+\delta \alpha}^{\dagger} t_{\delta}^{\alpha \beta} c_{j \beta} \mathcal{P}_{1} \sum_{j} \sum_{\delta} c_{j+\delta \alpha}^{\dagger} t_{\delta}^{\alpha \beta} c_{j \beta} .
$$

We evaluate this by choosing a site $j$ and summing over all virtual processes which go from $j$ to $j+\delta$ and back. Summing over all these terms exactly once will reproduce the superexchange model without double counting. This produces

$$
H_{\mathrm{eff}}=-\frac{1}{U} \sum_{j \delta} c_{j}^{\dagger}\left[t_{-\delta}+i \lambda_{-\delta} \cdot \sigma\right] c_{j+\delta} c_{j+\delta}^{\dagger}\left[t_{\delta}+i \lambda_{\delta} \cdot \sigma\right] c_{j},
$$

where the spin indices are suppressed, but understood to act according to usual matrix multiplication. The Hamiltonian is understood as being evaluated on the half-filling space, in which the projectors become redundant and are dropped. We evaluate this by first focusing on a single bond, where we have the term

$$
H_{12}=-\frac{1}{U} c_{1}^{\dagger}(t-i \lambda \cdot \sigma) c_{2} c_{2}^{\dagger}(t+i \lambda \cdot \sigma) c_{1} .
$$

We can evaluate this by rotating to the $\lambda$ axis, such that we have

$$
H_{12}=-\frac{1}{U} c_{1}^{\dagger}\left(t-i \lambda \sigma_{3}\right) c_{2} c_{2}^{\dagger}\left(t+i \lambda \sigma_{3}\right) c_{1} .
$$

There are now three terms; the regular superexchange term

$$
H_{12}^{0}=-\frac{t^{2}}{U} c_{1}^{\dagger} c_{2} c_{2}^{\dagger} c_{1}
$$

the antisymmetric DM exchange,

$$
H_{12}^{1}=-i \frac{t \lambda}{U}\left(c_{1}^{\dagger} c_{2} c_{2}^{\dagger} \sigma_{3} c_{1}-c_{1}^{\dagger} \sigma_{3} c_{2} c_{2}^{\dagger} c_{1}\right)
$$

and the symmetric anisotropy

$$
H_{12}^{2}=-\frac{\lambda^{2}}{U} c_{1}^{\dagger} \sigma_{3} c_{2} c_{2}^{\dagger} \sigma_{3} c_{1}
$$

Up to constants which are trivial in the half-filling sector, these can be evaluated to be

$$
\begin{aligned}
& H_{12}^{0}=2 \frac{t^{2}}{U} \hat{\mathbf{S}}_{1} \cdot \hat{\mathbf{S}}_{2}, \\
& H_{12}^{1}=-2 i \frac{t \lambda}{U}\left[\hat{S}_{1}^{+} \hat{S}_{2}^{-}-\hat{S}_{1}^{-} \hat{S}_{2}^{+}\right], \\
& H_{12}^{2}=2 \frac{\lambda^{2}}{U}\left(\hat{S}_{1}^{3} \hat{S}_{2}^{3}-\frac{1}{2} \hat{S}_{1}^{+} \hat{S}_{2}^{-}-\frac{1}{2} \hat{S}_{1}^{-} \hat{S}_{2}^{+}\right) .
\end{aligned}
$$

These are expressed in a covariant manner in terms of the spin-orbit vector as

$$
\begin{aligned}
& H_{12}^{0}=2 \frac{t^{2}}{U} \hat{\mathbf{S}}_{1} \cdot \hat{\mathbf{S}}_{2}, \\
& H_{12}^{1}=-4 \frac{t}{U} \lambda \cdot\left(\hat{\mathbf{S}}_{1} \times \hat{\mathbf{S}}_{2}\right), \\
& H_{12}^{2}=2 \frac{1}{U}\left[2\left(\lambda \cdot \hat{\mathbf{S}}_{1}\right)\left(\lambda \cdot \hat{\mathbf{S}}_{2}\right)-\lambda^{2} \hat{\mathbf{S}}_{1} \cdot \hat{\mathbf{S}}_{2}\right] .
\end{aligned}
$$

This then brings us to the full superexchange Hamiltonian:

$$
\begin{aligned}
H= & \sum_{j \delta} \frac{2\left(t^{2}-\lambda_{\delta}^{2}\right)}{U} \hat{\mathbf{S}}_{j} \cdot \hat{\mathbf{S}}_{j+\delta}-\frac{4 t}{U} \lambda_{\delta} \cdot\left(\hat{\mathbf{S}}_{j} \times \hat{\mathbf{S}}_{j+\delta}\right) \\
& +\frac{4}{U}\left(\lambda_{\delta} \cdot \hat{\mathbf{S}}_{j}\right)\left(\lambda_{\delta} \cdot \hat{\mathbf{S}}_{j+\delta}\right) .
\end{aligned}
$$

Using the Rashba spin-orbit texture amounts to substituting $\lambda_{\delta}=(-1)^{\ell} \lambda \delta \times \mathbf{e}_{z}$, with $\ell=u, d$ indexing the layer of the YBCO bilayer unit cell, as in the main text. In fact, each bond is counted twice in this expression such that if we perform the sum over bonds we must double the exchange interactions.

\section{Phonon coupling}

We now derive the coupling of the phonon displacement to the spin system by downfolding the electron-phonon Hamiltonian in the same manner. We include the spin-orbit and magnetostrictive couplings by taking

$$
t \rightarrow t+\alpha Q, \quad \lambda \rightarrow \lambda+\kappa Q
$$

in the above formulas and expanding to linear order in $Q$. To leading order, this leads to a spin-phonon interaction for each pair of sites 1,2 of

$$
\begin{aligned}
H_{\mathrm{int}}= & \frac{8 t \alpha}{U} \hat{Q}_{12} \hat{\mathbf{S}}_{1} \cdot \hat{\mathbf{S}}_{2}-\frac{8 \alpha}{U} \hat{Q}_{12} \lambda \cdot \hat{\mathbf{S}}_{1} \times \hat{\mathbf{S}}_{2} \\
& -\frac{8 t \kappa}{U \lambda} \hat{Q}_{12} \lambda \cdot \hat{\mathbf{S}}_{1} \times \hat{\mathbf{S}}_{2}-\frac{8 \lambda \kappa}{U} \hat{Q}_{12} \mathbf{S}_{1} \cdot \mathbf{S}_{2} \\
& +\frac{16 \kappa}{U \lambda} \hat{Q}_{12} \lambda \cdot \hat{\mathbf{S}}_{1} \lambda \cdot \hat{\mathbf{S}}_{2} .
\end{aligned}
$$

The first two terms come from linear variation of the scalar hopping $t$, while the last three come from the linear variation in the spin-orbit coupling. We anticipate the hierarchy of coupling constants $8 t \alpha / U \gg 8 \lambda \alpha / U \gtrsim 8 t \kappa / U \gg 8 \lambda \kappa / U$.

\section{APPENDIX B: EASY-PLANE MODEL}

Here we show how to diagonalize the easy-plane spin-wave Hamiltonian. We assume a classical Néel order which lies in the $x-y$ plane at an angle $\theta$ from the $x$ axis, and further 
choose the $x$ and $y$ axes to align with the $a$ and $b$ crystallographic axes, respectively. We then introduce the 1,2,3 coordinate system, such that

$$
\mathbf{e}_{3}=\cos \theta \mathbf{e}_{x}+\sin \theta \mathbf{e}_{y}
$$

defines the axis along which the Néel order lies. Then we choose the transverse 1 and 2 directions such that

$$
\mathbf{e}_{1} \times \mathbf{e}_{2}=\mathbf{e}_{3},
$$

i.e., the coordinate system is right-handed. One such choice is

$$
\begin{aligned}
& \mathbf{e}_{1}=\mathbf{e}_{z}, \\
& \mathbf{e}_{2}=\sin \theta \mathbf{e}_{x}-\cos \theta \mathbf{e}_{y}, \\
& \mathbf{e}_{3}=\cos \theta \mathbf{e}_{x}+\sin \theta \mathbf{e}_{y} .
\end{aligned}
$$

In this basis, we write the Holstein-Primakoff expansion as

$$
\hat{S}_{j}^{3}= \begin{cases}\frac{1}{2}-a_{j}^{\dagger} a_{j} & j \in A \\ b_{j}^{\dagger} b_{j}-\frac{1}{2} & j \in B .\end{cases}
$$

On the two sublattices, we then have a linear spin-wave expansion of

$$
\hat{S}_{j}^{+}=\hat{S}_{j}^{1}+i \hat{S}_{j}^{2}= \begin{cases}a_{j} & j \in A \\ b_{j}^{\dagger} & j \in B .\end{cases}
$$

The Hamiltonian can be then written in terms of the Holstein-Primakoff bosons as

$$
\begin{aligned}
H_{0}= & \sum_{\mathbf{q}}\left(a_{\mathbf{q}}^{\dagger}, b_{-\mathbf{q}}\right)\left(\begin{array}{cc}
2 J & (2 J-\Gamma) \gamma_{\mathbf{q}}^{s} \\
(2 J-\Gamma) \gamma_{\mathbf{q}}^{s} & 2 J
\end{array}\right)\left(\begin{array}{c}
a_{\mathbf{q}} \\
b_{-\mathbf{q}}^{\dagger}
\end{array}\right) \\
& -\sum_{\mathbf{q}} \Gamma \gamma_{\mathbf{q}}^{s}\left(a_{\mathbf{q}}^{\dagger} b_{\mathbf{q}}+a_{\mathbf{q}} b_{\mathbf{q}}^{\dagger}\right) .
\end{aligned}
$$

Next, we introduce the four-component Nambu spinor,

$$
\Psi_{\mathbf{q}}=\left(\begin{array}{c}
a_{\mathbf{q}} \\
b_{\mathbf{q}} \\
a_{-\mathbf{q}}^{\dagger} \\
b_{-\mathbf{q}}^{\dagger}
\end{array}\right),
$$

and the associated Nambu Pauli matrices $\tau_{a}$ (which act on the $\left(X, X^{\dagger}\right)$ doublet), as well as the sublattice Pauli matrices $\zeta_{a}$ [which act on the $(a, b)$ doublet]. We then have

$$
H=\frac{1}{2} \sum_{\mathbf{q}} \Psi_{\mathbf{q}}^{\dagger} \mathbb{M}_{\mathbf{q}} \Psi_{\mathbf{q}},
$$

with the quadratic form

$$
\mathbb{M}_{\mathbf{q}}=\left(2 J-\Gamma \gamma_{\mathbf{q}}^{s} \zeta_{1}\right)+\tau_{1}\left(2 J-\Gamma \gamma_{\mathbf{q}}^{s} \zeta_{1}\right)
$$

The central object of interest is the magnon Green's function, which is obtained by diagonalizing the kernel:

$$
\mathbb{D}^{-1}(q)=i \omega_{m} \tau_{3}-\mathbb{M}_{\mathbf{q}} .
$$

This can be diagonalized by first going to the eigenbasis of $\zeta_{1}$, which diagonalizes the sublattice eigenvalue $\zeta= \pm 1$. We then diagonalize the Nambu matrices to obtain the magnon dispersion relation:

$$
\Omega_{\mathbf{p} \zeta}=\sqrt{\left(2 J-\zeta \Gamma \gamma_{\mathbf{p}}^{s}\right)^{2}-(2 J-\Gamma)^{2}\left(\gamma_{\mathbf{p}}^{s}\right)^{2}} .
$$

While the two magnon bands are degenerate for the Heisenberg model, they now become split in the presence of anisotropy. At long wavelengths, one is gapped with gap

$$
\Omega_{\mathbf{p},-} \sim \sqrt{8 J \Gamma}+\cdots,
$$

while the other one remains gapless, with a spin-wave velocity of

$$
\Omega_{\mathbf{p},+} \sim \sqrt{2 J(2 J-\Gamma)}|\mathbf{p}| .
$$

We now determine the coupling to the electric field through the DM-type interaction for the case of Néel order in the $x-y$ plane. We consider the coupling

$$
H_{\mathrm{int}}=\sum_{j \in A, \boldsymbol{\delta}} g \hat{E}_{j, j+\delta}^{z}\left(\mathbf{e}_{z} \times \boldsymbol{\delta}\right) \cdot\left(\hat{\mathbf{S}}_{j} \times \hat{\mathbf{S}}_{j+\delta}\right) .
$$

We first express this in momentum space and separate out the linear and quadratic contributions as

$$
\begin{aligned}
& H_{\mathrm{int}}^{(2)}=\sum_{\mathbf{q}} g \hat{E}_{\mathbf{q}}^{z} \sum_{\delta}\left[\left(\mathbf{e}_{z} \times \boldsymbol{\delta}\right) \cdot \mathbf{e}_{2}\right] e^{i \frac{\mathbf{q} \cdot \boldsymbol{\delta}}{2}}\left(\hat{\mathbf{S}}_{j} \times \hat{\mathbf{S}}_{j+\boldsymbol{\delta}}\right)_{2, \mathbf{q}}, \\
& H_{\mathrm{int}}^{(3)}=\sum_{\mathbf{q}} g \hat{E}_{\mathbf{q}}^{z} \sum_{\delta}\left[\left(\mathbf{e}_{z} \times \boldsymbol{\delta}\right) \cdot \mathbf{e}_{3}\right] e^{i \frac{\mathbf{q} \cdot \boldsymbol{\delta}}{2}}\left(\hat{\mathbf{S}}_{j} \times \hat{\mathbf{S}}_{j+\boldsymbol{\delta}}\right)_{3, \mathbf{q}},
\end{aligned}
$$

with

$$
\left(\hat{\mathbf{S}}_{j} \times \hat{\mathbf{S}}_{j+\delta}\right)_{\mathbf{q}}=\sum_{j} \frac{e^{i \mathbf{q} \cdot \mathbf{R}_{j}}}{\sqrt{\operatorname{Vol} .}}\left(\hat{\mathbf{S}}_{j} \times \hat{\mathbf{S}}_{j+\delta}\right) .
$$

We find the resulting interaction of $H_{\text {int }}=H_{\text {int }}^{(2)}+H_{\text {int }}^{(3)}$ with

$$
\begin{aligned}
H_{\mathrm{int}}^{(2)}= & \sum_{\mathbf{p}} \frac{i g}{2} \hat{E}_{-\mathbf{p}}^{z}\left(\cos \theta \sin \frac{p_{x}}{2}+\sin \theta \sin \frac{p_{y}}{2}\right) \\
& \times\left(a_{\mathbf{q}}-b_{\mathbf{q}}\right)+\text { H.c., } \\
H_{\mathrm{int}}^{(3)}= & \sum_{\mathbf{q}, \mathbf{p}} \frac{g \hat{E}_{-\mathbf{q}}^{z}}{\sqrt{\mathrm{Vol}}}\left(\sin \theta \sin p_{x}-\cos \theta \sin p_{y}\right) \\
& \times a_{\mathbf{p}-\frac{\mathbf{q}}{2}}^{\dagger} b_{-\mathbf{p}-\frac{\mathbf{q}}{2}}^{\dagger}+\text { H.c. },
\end{aligned}
$$

and, in particular, if we project onto $\mathbf{q}=0$ for the electric field, we find the first term vanishes linearly with $\mathbf{q}$, while the second term recovers a finite homogeneous value.

\section{APPENDIX C: BILAYER HAMILTONIAN}

Here we calculate the spin-wave Hamiltonian for the bilayer system, assuming Néel order lies in the $a-b$ plane. We use the same 1,2,3 coordinates which were introduced in Appendix B for the case of easy-plane Néel order. The only further complication is due to the bilayer structure.

We focus on the case of the easy-plane Néel order since, following Ref. [153], we expect the in-plane Néel order to be stabilized by the frustrated anisotropies. Heuristically, this can be understood from the fact that, while different bond directions in the $a-b$ plane frustrate the anisotropies of each other, when the spin lies in the $x-y$ plane, it gains some anisotropy energy. On the other hand, when it points along $z$ it gains no anisotropy energy. Therefore, the in-plane order is stabilized. We would further expect that, because spin-rotation symmetry is broken explicitly by the frustrated spin-orbit coupling, a similar order-by-disorder mechanism ultimately 
opens a spin-wave gap even though classically the frustration produces a rotationally invariant interaction.

The only difference from the single-layer case is that we must also alternate the sublattice between the two bilayers, so we write the Holstein-Primakoff expansion as

$$
\hat{S}_{j \ell}^{3}= \begin{cases}\frac{1}{2}-a_{j \ell}^{\dagger} a_{j \ell} & (j, \ell) \in A \\ b_{j \ell}^{\dagger} b_{j \ell}-\frac{1}{2} & (j, \ell) \in B .\end{cases}
$$

We use the notation $(j, \ell) \in A, B$ to indicate that the sites on the $A$ sublattice in the upper layer are in the $B$ sublattice in the lower layer, and vice versa. On the two sublattices we have linear spin-wave expansions of

$$
\hat{S}_{j \ell}^{+}=\hat{S}_{j \ell}^{1}+i \hat{S}_{j \ell}^{2}= \begin{cases}a_{j \ell} & (j, \ell) \in A \\ b_{j \ell}^{\dagger} & (j, \ell) \in B .\end{cases}
$$

We now compute all the needed operators in terms of the local axes, expand in terms of the Holstein-Primakoff bosons, and express them in momentum space.

\section{Interlayer exchange}

We first express the interlayer coupling in terms of the local axes. This is easy because it is a spin scalar and therefore is basis independent. As such, we have

$$
\begin{aligned}
\hat{\mathbf{S}}_{j u} \cdot \hat{\mathbf{S}}_{j d} & =\hat{S}_{j u}^{3} \hat{S}_{j d}^{3}+\frac{1}{2} \hat{S}_{j u}^{+} \hat{S}_{j d}^{-}+\frac{1}{2} \hat{S}_{j u}^{-} \hat{S}_{j d}^{+} \\
& = \begin{cases}\frac{1}{2}\left[a_{j u}^{\dagger} a_{j u}+b_{j d}^{\dagger} b_{j d}+a_{j u} b_{j d}+a_{j u}^{\dagger} b_{j d}^{\dagger}\right] & j \in A \\
\frac{1}{2}\left[b_{j u}^{\dagger} b_{j u}+a_{j d}^{\dagger} a_{j d}+b_{j u} a_{j d}+b_{j u}^{\dagger} a_{j d}^{\dagger}\right] & j \in B,\end{cases}
\end{aligned}
$$

where the two cases correspond to the case where $j$ is such that $\mathbf{R}_{j}$ is in the $A$ sublattice on the upper layer (and therefore on the $B$ sublattice on the lower layer). This is the only term which couples the two layers. The remaining terms are now evaluated for the upper layer. To obtain the terms on the lower layer, we take $\lambda \rightarrow-\lambda$ and switch the two sublattices with each other.

To translate this to momentum space, we note that there is no dispersion from this term, only an ainterband coupling. We then obtain

$$
\begin{aligned}
H_{u d}= & J_{u d} \sum_{j \in A, B} \hat{\mathbf{S}}_{j u} \cdot \hat{\mathbf{S}}_{j d} \\
= & \frac{1}{2} J_{u d} \sum_{\mathbf{p} \ell}\left(a_{\mathbf{p} \ell}^{\dagger} a_{\mathbf{p} \ell}+b_{\mathbf{p} \ell}^{\dagger} b_{\mathbf{p} \ell}\right) \\
& +\frac{1}{2} J_{u d} \sum_{\mathbf{p}}\left(a_{\mathbf{p} u}^{\dagger}, b_{-\mathbf{p} u}\right)\left(\begin{array}{ll}
0 & 1 \\
1 & 0
\end{array}\right)\left(\begin{array}{c}
a_{\mathbf{p} d} \\
b_{-\mathbf{p} d}^{\dagger}
\end{array}\right) \\
& +\frac{1}{2} J_{u d} \sum_{\mathbf{p}}\left(a_{\mathbf{p} d}^{\dagger}, b_{-\mathbf{p} d}\right)\left(\begin{array}{ll}
0 & 1 \\
1 & 0
\end{array}\right)\left(\begin{array}{c}
a_{\mathbf{p} u} \\
b_{-\mathbf{p} u}^{\dagger}
\end{array}\right) .
\end{aligned}
$$

\section{Scalar exchange}

We now evaluate the scalar exchange interaction for each layer. Again, this is simplified since it is invariant upon changing to the local axes. The sum over all neighboring bonds is implemented by summing over $j \in A$ and all the $\delta$ vectors. We then have

$$
\hat{\mathbf{S}}_{j \ell} \cdot \hat{\mathbf{S}}_{j+\delta \ell}=\frac{1}{2}\left[a_{j \ell}^{\dagger} a_{j \ell}+b_{j+\delta \ell}^{\dagger} b_{j+\delta \ell}+a_{j \ell} b_{j+\delta \ell}+a_{j \ell}^{\dagger} b_{j+\delta \ell}^{\dagger}\right]
$$

for the scalar term, which is valid for both layers, provided we are careful to only sum over sites in the $A$ sublattice, which are translated relative to each other between each respective layer. We translate this into momentum space to find the Hamiltonian

$$
H_{0}=2 J_{0} \sum_{\ell} \sum_{\mathbf{p}}\left[a_{\mathbf{p} \ell}^{\dagger} a_{\mathbf{p} \ell}+b_{\mathbf{p} \ell}^{\dagger} b_{\mathbf{p} \ell}+\gamma_{\mathbf{p}} a_{\mathbf{p} \ell} b_{-\mathbf{p} \ell}+\gamma_{\mathbf{p}} a_{\mathbf{p} \ell}^{\dagger} b_{-\mathbf{p} \ell}^{\dagger}\right]
$$

with usual form factor $\gamma_{\mathbf{p}}=\frac{1}{2}\left(\cos p_{x}+\cos p_{y}\right)$ and $J_{0}=4\left(t^{2}-\lambda^{2}\right) / U$ the scalar exchange.

\section{Vector exchange}

The vector exchange is obtained by expanding in the $1,2,3$ basis as

$$
\hat{\mathbf{S}}_{j} \times \hat{\mathbf{S}}_{j+\delta}=\mathbf{e}_{3} \frac{\hat{S}_{j}^{-} \hat{S}_{j+\delta}^{+}-\hat{S}_{j}^{+} \hat{S}_{j+\delta}^{-}}{2 i}+\hat{S}_{j}^{3}\left(i \mathbf{e}_{+} \hat{S}_{j+\delta}^{+}-i \mathbf{e}_{-} \hat{S}_{j+\delta}^{-}\right)-\left(i \mathbf{e}_{+} \hat{S}_{j}^{+}-i \mathbf{e}_{-} \hat{S}_{j}^{-}\right) \hat{S}_{j+\delta}^{3},
$$

with $\mathbf{e}_{ \pm}=\frac{1}{2}\left(\mathbf{e}_{1} \mp i \mathbf{e}_{2}\right)$. We expand this operator up to quadratic order in the Holstein-Primakoff bosons. We obtain

$$
\hat{\mathbf{S}}_{j} \times \hat{\mathbf{S}}_{j+\delta}=\mathbf{e}_{3} \frac{a_{j}^{\dagger} b_{j+\delta}^{\dagger}-a_{j} b_{j+\delta}}{2 i}+\frac{1}{2}\left(i \mathbf{e}_{+} b_{j+\delta}^{\dagger}-i \mathbf{e}_{-} b_{j+\delta}\right)+\frac{1}{2}\left(i \mathbf{e}_{+} a_{j}-i \mathbf{e}_{-} a_{j}^{\dagger}\right) .
$$

In particular, we need the projection of this operator onto the vector $\mathbf{e}_{z} \times \delta$. We obtain

$$
\hat{\mathbf{S}}_{j} \times \hat{\mathbf{S}}_{k} \cdot\left(\mathbf{e}_{z} \times \boldsymbol{\delta}\right)= \begin{cases}\sin \theta\left(\frac{a_{j}^{\dagger} b_{j+\delta}^{\dagger}-a_{j} b_{j+\delta}}{2 i}\right)-\frac{\cos \theta}{4}\left(b_{j+\delta}^{\dagger}+b_{j+\delta}+a_{j}+a_{j}^{\dagger}\right) & \boldsymbol{\delta}=\mathbf{e}_{x} \\ -\cos \theta\left(\frac{a_{j}^{\dagger} b_{j+\delta}^{\dagger}-a_{j} b_{j+\delta}}{2 i}\right)-\frac{\sin \theta}{4}\left(b_{j+\delta}^{\dagger}+b_{j+\delta}+a_{j}+a_{j}^{\dagger}\right) & \boldsymbol{\delta}=\mathbf{e}_{y} .\end{cases}
$$


We have suppressed the layer index for brevity (since at the moment the result is the same for both). To express this in momentum space, we note that the linear term will cancel since the sum involves terms with both $\delta$ and $-\boldsymbol{\delta}$. This leaves

$$
\begin{aligned}
H_{1}=-\sum_{j \in A, \ell}(-1)^{\ell} \frac{8 t \lambda}{U}[ & \sin \theta\left(\frac{a_{j \ell}^{\dagger} b_{j+x \ell}^{\dagger}-a_{j \ell} b_{j+x \ell}}{2 i}-\frac{a_{j \ell}^{\dagger} b_{j-x \ell}^{\dagger}-a_{j \ell} b_{j-x \ell}}{2 i}\right) \\
& \left.-\cos \theta\left(\frac{a_{j \ell}^{\dagger} b_{j+y \ell}^{\dagger}-a_{j \ell} b_{j+y \ell}}{2 i}-\frac{a_{j \ell}^{\dagger} b_{j-y \ell}^{\dagger}-a_{j \ell} b_{j-y \ell}}{2 i}\right)\right] .
\end{aligned}
$$

Going to momentum space, we find

$$
H_{1}=\sum_{\ell \mathbf{p}}-(-1)^{\ell} \frac{8 t \lambda}{U}\left[\sin \theta \sin p_{x}-\cos \theta \sin p_{y}\right]\left(a_{\mathbf{p} \ell} b_{-\mathbf{p} \ell}+b_{-\mathbf{p} \ell}^{\dagger} a_{\mathbf{p} \ell}^{\dagger}\right) .
$$

\section{Tensor exchange}

The tensor exchange is

$$
\hat{\mathbf{S}}_{j} \cdot\left(\mathbf{e}_{z} \times \boldsymbol{\delta}\right)\left(\mathbf{e}_{z} \times \boldsymbol{\delta}\right) \cdot \hat{\mathbf{S}}_{k}= \begin{cases}\left(-\cos \theta \hat{S}_{j}^{2}+\sin \theta \hat{S}_{j}^{3}\right)\left(-\cos \theta \hat{S}_{k}^{2}+\sin \theta \hat{S}_{k}^{3}\right) & \boldsymbol{\delta}=\mathbf{e}_{x} \\ \left(-\sin \theta \hat{S}_{j}^{2}-\cos \theta \hat{S}_{j}^{3}\right)\left(-\sin \theta \hat{S}_{k}^{2}-\cos \theta \hat{S}_{k}^{3}\right) & \boldsymbol{\delta}=\mathbf{e}_{y} .\end{cases}
$$

Next, we express this in terms of the angular momentum ladder operators $S^{ \pm}=S^{1} \pm i S^{2}$. We have

$$
\hat{\mathbf{S}}_{j \ell} \cdot\left(\mathbf{e}_{z} \times \boldsymbol{\delta}\right)\left(\mathbf{e}_{z} \times \boldsymbol{\delta}\right) \cdot \hat{\mathbf{S}}_{j+\delta \ell}=\left\{\begin{array}{l}
\left(-\cos \theta \frac{\hat{S}_{j \ell}^{+}-\hat{S}_{-\ell}^{-}}{2 i}+\sin \theta \hat{S}_{j \ell}^{3}\right)\left(-\cos \theta \frac{\hat{S}_{j+\delta \ell}^{+}-\hat{S}_{j+\delta \ell}^{-}}{2 i}+\sin \theta \hat{S}_{j+\delta \ell}^{3}\right) \quad \boldsymbol{\delta}=\mathbf{e}_{x} \\
\left(-\sin \theta \frac{\hat{S}_{j \ell}^{+}-\hat{S}_{j \ell}^{-}}{2 i}-\cos \theta \hat{S}_{j \ell}^{3}\right)\left(-\sin \theta \frac{\hat{S}_{j+\delta \ell}^{+}-\hat{S}_{j+\delta \ell}^{-}}{2 i}-\cos \theta \hat{S}_{j+\delta \ell}^{3}\right) \quad \boldsymbol{\delta}=\mathbf{e}_{y} .
\end{array}\right.
$$

This is now expressed in terms of the Holstein-Primakoff bosons, up to quadratic order. This is slightly more complicated because there are cross terms between $S^{3}$, which contains the zeroth- and second-order terms, and the transverse terms which contain linear terms. We drop terms which are overall constants, such that

$$
\begin{aligned}
\hat{\mathbf{S}}_{j \ell} & \cdot\left(\mathbf{e}_{z} \times \boldsymbol{\delta}\right)\left(\mathbf{e}_{z} \times \boldsymbol{\delta}\right) \cdot \hat{\mathbf{S}}_{j+\delta \ell} \\
\quad & = \begin{cases}-\frac{1}{4} \cos ^{2} \theta\left(a_{j \ell}-a_{j \ell}^{\dagger}\right)\left(b_{j+\delta \ell}^{\dagger}-b_{j+\delta \ell}\right)+\frac{1}{2} \sin ^{2} \theta\left(a_{j \ell}^{\dagger} a_{j \ell}+b_{j+\delta \ell}^{\dagger} b_{j+\delta \ell}\right)+\frac{1}{4 i} \sin \theta \cos \theta\left(a_{j \ell}-a_{j \ell}^{\dagger}-b_{j+\delta \ell}^{\dagger}+b_{j+\delta \ell}\right) & \boldsymbol{\delta}=\mathbf{e}_{x} \\
-\frac{1}{4} \sin ^{2} \theta\left(a_{j \ell}-a_{j \ell}^{\dagger}\right)\left(b_{j+\delta \ell}^{\dagger}-b_{j+\delta \ell}\right)+\frac{1}{2} \cos ^{2} \theta\left(a_{j \ell}^{\dagger} a_{j \ell}+b_{j+\delta \ell}^{\dagger} b_{j+\delta \ell}\right)-\frac{1}{4 i} \sin \theta \cos \theta\left(a_{j \ell}-a_{j \ell}^{\dagger}-b_{j+\delta \ell}^{\dagger}+b_{j+\delta \ell}\right) & \boldsymbol{\delta}=\mathbf{e}_{y} .\end{cases}
\end{aligned}
$$

We now pass to momentum space again. Just like in the vector coupling, the linear term will cancel because the $x$ and $y$ anisotropies enter with opposite signs in the linear part. Then we observe that of the two quadratic terms, the one which conserves boson number, when summed over the four different bond values, will give $a_{j \ell}^{\dagger} a_{j \ell}+b_{j+\delta \ell}^{\dagger} b_{j+\delta}$, independent of the Néel ordering vector. Therefore, there is one contribution which is simply

$$
H_{2,1}=\frac{8 \lambda^{2}}{U} \sum_{\mathbf{p} \ell} a_{\mathbf{p} \ell}^{\dagger} a_{\mathbf{p} \ell}+b_{\mathbf{p} \ell}^{\dagger} b_{\mathbf{p} \ell}
$$

The other contribution comes from the first term and in real space reads

$$
H_{2,2}=-\frac{2 \lambda^{2}}{U} \sum_{j \in A, \ell}\left[\cos ^{2} \theta\left(a_{j \ell}-a_{j \ell}^{\dagger}\right)\left(b_{j+x \ell}^{\dagger}-b_{j+x \ell}+b_{j-x \ell}^{\dagger}-b_{j-x \ell}\right)+\sin ^{2} \theta\left(a_{j \ell}-a_{j \ell}^{\dagger}\right)\left(b_{j+y \ell}^{\dagger}-b_{j+y \ell}+b_{j-y \ell}^{\dagger}-b_{j-y \ell}\right)\right] .
$$

In momentum space, this reads

$$
H_{2,2}=-\frac{1}{2} \Gamma \sum_{\mathbf{p} \ell}\left(b_{\mathbf{p} \ell}^{\dagger}-b_{-\mathbf{p} \ell}\right)\left(a_{\mathbf{p} \ell}-a_{-\mathbf{p} \ell}^{\dagger}\right)\left(\cos p_{x} \cos ^{2} \theta+\cos p_{y} \sin ^{2} \theta\right),
$$

with $\Gamma=8 \lambda^{2} / U$. 


\section{Full bilayer model}

We now present the full bilayer Hamiltonian in momentum space, to second order in the Holstein-Primakoff bosons. We have

$$
\begin{aligned}
H= & \frac{1}{2} J_{u d} \sum_{\mathbf{p}} a_{\mathbf{p} u}^{\dagger} b_{-\mathbf{p} d}^{\dagger}+a_{\mathbf{p} d}^{\dagger} b_{-\mathbf{p} u}^{\dagger}+b_{-\mathbf{p} d} a_{\mathbf{p} u}+b_{-\mathbf{p} u} a_{\mathbf{p} d}+\frac{1}{2} J_{u d} \sum_{\mathbf{p} \ell} a_{\mathbf{p} \ell}^{\dagger} a_{\mathbf{p} \ell}+b_{\mathbf{p} \ell}^{\dagger} b_{\mathbf{p} \ell} \\
& +\sum_{\ell \mathbf{p}}\left(a_{\mathbf{p} \ell}^{\dagger}, b_{-\mathbf{p} \ell}\right)\left(\begin{array}{cc}
2 J_{0}+\Gamma & 2 J_{0} \gamma_{\mathbf{p}}^{s}-D_{\ell} \gamma_{\mathbf{p}}^{p}(\theta)+\frac{1}{2} \Gamma \tilde{\gamma}_{\mathbf{p}}^{d}(\theta) \\
2 J_{0} \gamma_{\mathbf{p}}^{s}-D_{\ell} \gamma_{\mathbf{p}}^{p}(\theta)+\frac{1}{2} \Gamma \tilde{\gamma}_{\mathbf{p}}^{d}(\theta) & 2 J_{0}+\Gamma
\end{array}\right)\left(\begin{array}{c}
a_{\mathbf{p} \ell} \\
b_{-\mathbf{p} \ell}^{\dagger}
\end{array}\right) \\
& -\frac{1}{2} \sum_{\mathbf{p} \ell} \Gamma \tilde{\gamma}^{d}(\theta)\left(a_{\mathbf{p} \ell}^{\dagger} b_{\mathbf{p} \ell}+b_{\mathbf{p} \ell}^{\dagger} a_{\mathbf{p} \ell}\right) .
\end{aligned}
$$

Here we have introduced multiple form factors (which are dependent on the Néel order angle), which are defined as

$$
\begin{aligned}
\gamma_{\mathbf{p}}^{s} & =\frac{1}{2}\left(\cos p_{x}+\cos p_{y}\right), \\
\gamma_{\mathbf{p}}^{p}(\theta) & =\sin \theta \sin p_{x}-\cos \theta \sin p_{y}, \\
\tilde{\gamma}_{\mathbf{p}}^{d}(\theta) & =\cos p_{x} \cos ^{2} \theta+\cos p_{y} \sin ^{2} \theta=\gamma_{\mathbf{p}}^{s}+\cos 2 \theta \gamma_{\mathbf{p}}^{d}, \\
\gamma_{\mathbf{p}}^{d} & =\frac{1}{2}\left(\cos p_{x}-\cos p_{y}\right),
\end{aligned}
$$

and the exchange constants are, in terms of the Hubbard model parameters,

$$
\begin{aligned}
J_{0} & =4 \frac{t^{2}-\lambda^{2}}{U}, \\
D_{\ell} & =8 \frac{t \lambda}{U}(-1)^{\ell}, \\
\Gamma & =8 \frac{\lambda^{2}}{U} .
\end{aligned}
$$

It will also be relevant to include the matrix elements of the DM-type interaction with the electric field, which takes the form

$$
H_{\mathrm{int}}=\sum_{j \in A \delta \ell} g \hat{E}_{j, j+\delta}^{z}\left(\mathbf{e}_{z} \times \boldsymbol{\delta}\right) \cdot \hat{\mathbf{S}}_{j \ell} \times \hat{\mathbf{S}}_{j+\delta \ell} .
$$

This will yield the same result as previously, except we must sum over the layer index $\ell$. We thus find

$$
\begin{aligned}
H_{\mathrm{int}}^{(2)}= & \sum_{\mathbf{p} \ell} \hat{E}_{-\mathbf{p}}^{z} i \frac{g}{2}\left(\cos \theta \sin \frac{p_{x}}{2}+\sin \theta \sin \frac{p_{y}}{2}\right) \\
& \times\left(a_{\mathbf{p} \ell}-b_{\mathbf{p} \ell}\right)+\text { H.c., } \\
H_{\mathrm{int}}^{(3)}= & \sum_{\mathbf{q}, \mathbf{p} \ell} \frac{g \hat{E}_{\mathbf{q}}^{z}}{\sqrt{\operatorname{Vol}}}\left(\sin \theta \sin p_{x}-\cos \theta \sin p_{y}\right) \\
& \times a_{\mathbf{p}+\frac{\mathbf{q}}{2} \ell}^{\dagger} b_{-\mathbf{p}+\frac{\mathbf{q}}{2} \ell}^{\dagger}+\text { H.c. }
\end{aligned}
$$

We can verify that inversion takes $a_{\mathbf{q} u} \rightarrow a_{-\mathbf{q} d}$ and similarly for $b$, and also takes $Q_{\mathbf{q}} \rightarrow-Q_{-\mathbf{q}}$. Therefore, the interaction as written preserves inversion symmetry.

\section{Diagonalizing bilayer maodel}

The spin-wave Hamiltonian for the full bilayer system can be diagonalized exactly. Similar to the monolayer case, we introduce $\tau_{a}$ for Nambu space Pauli matrices and $\zeta_{a}$ for sublattice space Pauli matrices. We must now also introduce an additional layer space $l_{a}$ set of Pauli matrices. In terms of these, and the eight-component Nambu-sublattice-layer spinor $\Psi_{\mathrm{p}}$, we have the Hamiltonian

$$
H=\frac{1}{2} \sum_{\mathbf{p}} \Psi_{\mathbf{p}}^{\dagger} \hat{\mathbb{M}}_{\mathbf{p}} \Psi_{\mathbf{p}}
$$

with the matrix

$$
\begin{aligned}
\hat{\mathbb{M}}_{\mathbf{p}}= & \frac{1}{2} J_{u d}\left[1+\tau_{1} \zeta_{1} l_{1}\right]+2 J_{0}+\Gamma-\frac{1}{2} \Gamma\left(\gamma_{\mathbf{p}}^{s}+\cos 2 \theta \gamma_{\mathbf{p}}^{d}\right) \zeta_{1} \\
& +\left[2 J_{0} \gamma_{\mathbf{p}}^{s}+\frac{1}{2} \Gamma\left(\gamma_{\mathbf{p}}^{s}+\cos 2 \theta \gamma_{\mathbf{p}}^{d}\right)\right] \tau_{1} \zeta_{1}+D \gamma_{\mathbf{p}}^{p} \tau_{2} \zeta_{2} l_{3} .
\end{aligned}
$$

Our aim is to diagonalize the dynamical matrix, $\hat{\mathbb{K}}_{\mathbf{p}}=\tau_{3} \hat{\mathbb{M}}_{\mathbf{p}}$, which in turn can be used to compute the magnon Matsubara Green's function by inverting the kernel

$$
\hat{\mathbb{D}}_{\mathbf{p}}^{-1}=i \omega_{m} \tau_{3}-\hat{\mathbb{M}}_{\mathbf{p}}=\tau_{3}\left(i \omega_{m}-\hat{\mathbb{K}}_{\mathbf{p}}\right) .
$$

We begin by noting that the sublattice parity matrix $\Pi=\zeta_{1} l_{1}$ commutes with the Green's function and obeys $\Pi^{2}=1$. We therefore find two twofold degenerate representations, each of definite parity as determined by the eigenvalue of $\Pi= \pm 1$.

To finish diagonalizing the spin-wave kernel, we define $Z=\zeta_{1}$. In each subspace of $\Pi$, we have two basis states, which can be labeled by their eigenvalue of $Z$. For instance, for $\Pi=+1$ the two states are $|++\rangle$ and $|--\rangle$, with the first number labeling the eigenvalue of $\zeta_{1}=Z$, and the second labeling the eigenvalue of $l_{1}=\Pi \zeta_{1}$. The action of $\zeta_{2} l_{3}$ on these basis states can then be mapped onto the action of $-Y$, such that the dynamical matrix assumes the form

$$
\hat{\mathbb{K}}=\tau_{3}\left[a_{3}+b_{3} Z\right]+i \tau_{2}\left[a_{2}+b_{2} Z\right]+i \tau_{1} c Y .
$$

We know that the eigenvalues will come in particle-hole conjugate pairs, and we therefore consider squaring $\mathbb{K}$ and diagonalizing that matrix. We find

$$
\begin{aligned}
\hat{\mathbb{K}}^{2}= & \left(a_{3}+b_{3} Z\right)^{2}-\left(a_{2}+b_{2} Z\right)^{2}-c^{2} \\
& +2 i \tau_{2} c b_{3} X+2 \tau_{3} c b_{2} X .
\end{aligned}
$$

This matrix is now reduced to a Clifford algebra form, with $Z, \tau_{2} X, \tau_{3} X$ forming a set of three anticommuting matrices which all square to one. We therefore find that the eigenvalues $\lambda$ of $\hat{\mathbb{K}}$ are

$$
\begin{aligned}
\lambda_{ \pm}= & E_{ \pm}^{2}=a_{3}^{2}+b_{3}^{2}-a_{2}^{2}-b_{2}^{2}-c^{2} \\
& \pm \sqrt{\left(2 a_{3} b_{3}-2 a_{2} b_{2}\right)^{2}-\left(2 c b_{3}\right)^{2}+\left(2 c b_{2}\right)^{2}} .
\end{aligned}
$$


It follows that the energy eigenvalues are

$$
E_{\zeta}=\sqrt{a_{3}^{2}+b_{3}^{2}-a_{2}^{2}-b_{2}^{2}-c^{2}-2 \zeta \sqrt{\left(a_{3} b_{3}-a_{2} b_{2}\right)^{2}-\left(c b_{3}\right)^{2}+\left(c b_{2}\right)^{2}},}
$$

assuming that the energies remain real, and therefore there is no dynamical instability. We have introduced the quantum number $\zeta= \pm$ here, which is chosen such that in the limit of $\mathbf{p} \rightarrow \mathbf{0}$, this coincides with the eigenvalue of $\zeta_{1}$, at least for a certain range of $\Gamma / J_{u d}$. The parameters in the above formula are

$$
\begin{aligned}
a_{3} & =2 J_{0}+\Gamma+\frac{1}{2} J_{u d}, \\
b_{3} & =-\frac{1}{2} \Gamma\left(\gamma_{\mathbf{p}}^{s}+\cos 2 \theta \gamma_{\mathbf{p}}^{d}\right), \\
a_{2} & =\frac{1}{2} J_{u d} \Pi, \\
b_{2} & =2 J_{0} \gamma_{\mathbf{p}}^{s}+\frac{1}{2} \Gamma\left(\gamma_{\mathbf{p}}^{s}+\cos 2 \theta \gamma_{\mathbf{p}}^{d}\right), \\
c & =D \gamma_{\mathbf{p}}^{p} .
\end{aligned}
$$

In particular, we have the following results as $\mathbf{p} \rightarrow \mathbf{0}$. First, as $\mathbf{p} \rightarrow \mathbf{0}$ we find that the quantum numbers become identified as the eigenvalue of $\zeta_{1}$, so we will label them as $\zeta= \pm$, even though this assignment is only accurate strictly at $\mathbf{p}=\mathbf{0}$. Second, we find the energies at $\mathbf{p}=\mathbf{0}$ of

$$
E_{\Pi= \pm, \zeta= \pm}(\mathbf{p}=\mathbf{0})=\sqrt{\left(2 J_{0}+\Gamma+\frac{1}{2} J_{u d}-\zeta \frac{1}{2} \Gamma\right)^{2}-\left(2 J_{0}+\frac{1}{2} \Gamma+\zeta \Pi J_{u d} / 2\right)^{2}}
$$

This simplifies to yield

$$
\begin{aligned}
& E_{++}(0)=0 \\
& E_{+-}(0)=\sqrt{2\left(2 J_{0}+\Gamma\right)\left(\Gamma+J_{u d}\right)}, \\
& E_{-+}(0)=\sqrt{2\left(2 J_{0}+\Gamma / 2\right) J_{u d}}, \\
& E_{--}(0)=\sqrt{2\left(2 J_{0}+\Gamma+\frac{1}{2} J_{u d}\right) \Gamma .}
\end{aligned}
$$

The group velocity of the gapless pseudo-Goldstone mode is, within this approximation, given by the dispersion relation

$$
\begin{aligned}
E_{++}(\mathbf{p}) & =\sqrt{A_{x x} p_{x}^{2}+2 A_{x y} p_{x} p_{y}+A_{y y} p_{y}^{2}}, \\
A_{x x} & =\left(2 J_{0}+\frac{1}{2} \Gamma+\frac{1}{2} J_{u d}\right)\left(\Gamma \cos ^{2} \theta+J_{0}-\frac{2 D^{2} \sin ^{2} \theta}{\Gamma+J_{u d}}\right), \\
A_{x y} & =\left(2 J_{0}+\frac{1}{2} \Gamma+\frac{1}{2} J_{u d}\right) \frac{2 D^{2}}{\Gamma+J_{u d}} \sin \theta \cos \theta, \\
A_{y y} & =\left(2 J_{0}+\frac{1}{2} \Gamma+\frac{1}{2} J_{u d}\right)\left(\Gamma \sin ^{2} \theta+J_{0}-\frac{2 D^{2} \cos ^{2} \theta}{\Gamma+J_{u d}}\right) .
\end{aligned}
$$

This has a stability criterion that the quadratic form formed by the elements $A_{x x}, A_{x y}, A_{y y}$ be positive semidefinite. For $\theta=0$, this amounts to

$$
J_{u d} / J_{0}>4 \frac{D^{2}}{J_{0}^{2}}-\Gamma / J_{0}^{2} \sim 6(\lambda / t)^{2} .
$$

Therefore, for weak spin-orbit coupling (we take $\lambda / t \sim 0.025$ ), this is quite reasonable.

\section{Green's function}

We now obtain the magnon Green's function, which is used in the linear response calculations. Writing out the Nambu structure explicitly in block matrix form, the inverse Green's function takes the form

$$
\hat{\mathbb{D}}_{\mathbf{q}}^{-1}(z)=\left(\begin{array}{cc}
\mathbb{1} & 0 \\
0 & -\mathbb{1}
\end{array}\right)\left(\begin{array}{cc}
z-\hat{\mathbb{A}}_{\mathbf{q}} & -\left(\hat{\mathbb{B}}_{\mathbf{q}}-i \hat{\mathbb{C}}_{\mathbf{q}}\right) \\
\hat{\mathbb{B}}_{\mathbf{q}}+i \hat{\mathbb{C}}_{\mathbf{q}} & z+\hat{\mathbb{A}}_{\mathbf{q}}
\end{array}\right),
$$

with $z$ lying in the complex plane and $\hat{\mathbb{A}}, \hat{\mathbb{B}}, \hat{\mathbb{C}}$ matrices in the sublattice and bilayer subspaces. 
Provided certain conditions are satisfied by the matrices $\hat{\mathbb{A}}, \hat{\mathbb{B}}, \hat{\mathbb{C}}$, we can find the Green's function by explicitly performing the block matrix inversion procedure. We then find

$$
\begin{aligned}
\hat{\mathbb{D}}_{\mathbf{q}}(z)= & \left(\begin{array}{cc}
{\left[z-\hat{\mathbb{A}}_{\mathbf{q}}+\left(\hat{\mathbb{B}}_{\mathbf{q}}-i \hat{\mathbb{C}}_{\mathbf{q}}\right)\left(z+\hat{\mathbb{A}}_{\mathbf{q}}\right)^{-1}\left(\hat{\mathbb{B}}_{\mathbf{q}}+i \hat{\mathbb{C}}_{\mathbf{q}}\right)\right]^{-1}} & 0 \\
0 & {\left[z+\hat{\mathbb{A}}_{\mathbf{q}}+\left(\hat{\mathbb{B}}_{\mathbf{q}}+i \hat{\mathbb{C}}_{\mathbf{q}}\right)\left(z-\hat{\mathbb{A}}_{\mathbf{q}}\right)^{-1}\left(\hat{\mathbb{B}}_{\mathbf{q}}-i \hat{\mathbb{C}}_{\mathbf{q}}\right)\right]^{-1}}
\end{array}\right) \\
& \times\left(\begin{array}{cc}
\mathbb{1} & \left(\hat{\mathbb{B}}_{\mathbf{q}}-i \hat{\mathbb{C}}_{\mathbf{q}}\right)\left(z+\hat{\mathbb{A}}_{\mathbf{q}}\right)^{-1} \\
-\left(\hat{\mathbb{B}}_{\mathbf{q}}+i \hat{\mathbb{C}}_{\mathbf{q}}\right)\left(z-\hat{\mathbb{A}}_{\mathbf{q}}\right)^{-1} & \mathbb{1}
\end{array}\right)\left(\begin{array}{cc}
\mathbb{1} & 0 \\
0 & -\mathbb{1}
\end{array}\right) .
\end{aligned}
$$

We can then perform all the necessary analytical continuations.

\section{Photon-magnon coupling in bilayer model}

In this section, we derive the magnon-photon coupling in the bilayer model. Throughout this section, we assume the photonelectron coupling to of the scalar type $\kappa \sum_{j \in A, \delta, \ell} c_{j+\delta \ell}^{\dagger} \hat{E}_{j, j+\delta} c_{j \ell}$. In Appendix C 7 a, we derive the effective coupling between magnons and photons. Based on this coupling in Appendix C $7 \mathrm{~b}$, we derive the change in susceptibility due to magnons.

\section{a. Phonon-magnon coupling}

We now perform the adiabatic elimination of doublon states and derive the effective coupling between photon and magnon degrees of freedom. Analogously to Appendix $\mathrm{C}$ we find the scalar $H_{\mathrm{sc}}$ and vector $H_{\mathrm{vec}}$ terms. By expanding up to the linear order in $\kappa$, we find the scalar coupling term,

$$
H_{\mathrm{sc}}^{(2)} \approx \frac{8}{U} \frac{(2 t)}{\sqrt{M}} \kappa \sum_{\boldsymbol{k}, \boldsymbol{q}, \ell}(-1)^{\ell}\left(\hat{E}_{-\boldsymbol{q}} a_{\boldsymbol{q}-\boldsymbol{k}, \ell} b_{\boldsymbol{k}, \ell}+\hat{E}_{\boldsymbol{q}} a_{\boldsymbol{q}-\boldsymbol{k}, \ell}^{\dagger} b_{\boldsymbol{k}, \ell}^{\dagger}\right) \psi_{\boldsymbol{k}-\frac{q}{2}}^{(s)}+\frac{8}{U} \frac{(2 t)}{\sqrt{M}} \kappa \sum_{\boldsymbol{k}, \boldsymbol{k}^{\prime}, \ell}(-1)^{\ell} \hat{E}_{\boldsymbol{q}}\left(b_{\boldsymbol{k}+\boldsymbol{q}, \ell}^{\dagger} b_{\boldsymbol{k}, \ell}+a_{\boldsymbol{k}+\boldsymbol{q}, \ell}^{\dagger} a_{\boldsymbol{k}, \ell}\right) \psi_{\boldsymbol{q}}^{(s)},
$$

where $\psi_{k}^{(s)}=\frac{1}{2}\left(\cos \left(k_{x}\right)+\cos \left(k_{y}\right)\right)$. Analogously, we find quadratic the vector term,

$$
\begin{aligned}
H_{\mathrm{vec}}^{(2)} & =-i \frac{2 \lambda}{U} \sum_{j, \delta, \ell}(-1)^{\ell}\left(\hat{c}_{j, \ell}^{\dagger} \hat{c}_{j+\delta, \ell} \hat{c}_{j+\delta, \ell}^{\dagger} \hat{E}_{j, j+\delta, \ell} \cdot \boldsymbol{\sigma} \hat{c}_{j, \ell}-\hat{c}_{j, \ell}^{\dagger} \hat{E}_{j, j+\delta, \ell} \cdot \boldsymbol{\sigma} \hat{c}_{j+\delta, \ell} \hat{c}_{j+\delta, \ell}^{\dagger} \hat{c}_{j, \ell}\right) \\
& =\frac{8 \lambda}{U} \frac{1}{\sqrt{M}} \kappa \sum_{\boldsymbol{q}, q^{\prime}}(-1)^{\ell}\left(\hat{E}_{\boldsymbol{q}^{\prime}+q} a_{\boldsymbol{q}^{\prime}, \ell}^{\dagger} \ell_{\boldsymbol{q}, \ell}^{\dagger}+\hat{E}_{\boldsymbol{q}^{\prime}+\boldsymbol{q}}^{*} a_{\boldsymbol{q}^{\prime}, \ell} b_{\boldsymbol{q}, \ell}\right)\left(\sin \frac{\left(q_{x}^{\prime}-q_{x}\right)}{2} \sin \theta-\sin \frac{\left(q_{y}^{\prime}-q_{y}\right)}{2} \cos \theta\right),
\end{aligned}
$$

and linear terms:

$$
H_{\mathrm{vec}}^{(1)}=\frac{4 t \lambda i}{U} \sum_{\boldsymbol{q}, \ell}(-1)^{\ell} \hat{E}_{-\boldsymbol{q}}\left(b_{\boldsymbol{q}, \ell}-a_{\boldsymbol{q}, \ell}+b_{-\boldsymbol{q}, \ell}^{\dagger}-a_{-\boldsymbol{q}, \ell}^{\dagger}\right)\left(\cos \theta \sin \frac{q_{x}}{2}+\sin \frac{q_{y}}{2} \sin \theta\right) .
$$

Below, we derive the susceptibility change due to the presence of magnons.

\section{b. Susceptibility}

In this section, we provide technical details of calculation of susceptibility in Sec. V. We consider the bilayer model derived above with the anisotropy parameter $\Gamma$ and without spin-orbit coupling $\lambda$. With the free bilayer Hamiltonian $\hat{H}_{0}=$ $\frac{1}{2} \sum_{k} \Psi_{k}^{\dagger} \mathbb{M}_{k} \Psi_{k}$ and the photon-magnon term $\hat{\boldsymbol{E}}_{0} \hat{V}$ with $\hat{V}=\frac{1}{2} \sum_{k} \Psi_{k}^{\dagger} \mathbb{V}_{k} \Psi_{k}$, which we get from Eq. (C34) by setting the field momentum to zero. The Nambu matrices are

$$
\begin{gathered}
\mathbb{M}_{\boldsymbol{k}}=\left(2 J-\Gamma \psi_{k}^{(s)} \zeta_{1}\right)+(2 J-\Gamma) \psi_{k}^{(s)} \tau_{1} \zeta_{1}+\frac{J_{u d}}{2}\left(1+\tau_{1} \zeta_{1} l_{1}\right) \\
\mathbb{V}_{\boldsymbol{k}}=2 g^{\prime}\left(1+\psi_{k}^{(s)} \tau_{1} \zeta_{1}\right) l_{3}
\end{gathered}
$$

The imaginary-frequency susceptibility reads

$$
\chi\left(i \omega_{n}\right)=\int d \tau e^{i \omega_{n} \tau}\left\langle T_{\tau} \hat{V}(\tau) \hat{V}(0)\right\rangle=\frac{1}{\beta} \sum_{k} \sum_{m} \operatorname{Tr}\left\{\mathbb{G}_{k}\left(i \omega_{m}-i \omega_{n}\right) \mathbb{V}_{\boldsymbol{k}} \mathbb{G}_{\boldsymbol{k}}\left(i \omega_{m}\right) \mathbb{V}_{\boldsymbol{k}}\right\},
$$

where $T_{\tau}$ denotes the time ordering and in the last line we used Wick's theorem. We note that in Eq. (C35) the $1 / 4$ factor is canceled by two identical contributions to the susceptibility and due to the parity $(\boldsymbol{k} \rightarrow-\boldsymbol{k})$ symmetry. The unperturbed propagator reads

$$
\mathbb{G}_{\boldsymbol{k}}\left(i \omega_{m}\right)=\left(i \tau^{3} \omega_{m}-\mathbb{M}_{0}\right)^{-1}
$$


In the zero-temperature limit, we find

$$
\chi(i \Omega)=\sum_{k} \int \frac{d \omega}{2 \pi} \operatorname{Tr}\left\{\mathbb{G}_{k}(i \omega-i \Omega) \mathbb{V}_{k} \mathbb{G}_{k}(i \omega) \mathbb{V}_{k}\right\}
$$

To derive the expression for susceptibility of arbitrary $\Gamma$, it is convenient to parameterized the matrix $\mathbb{M}_{k}$ as follows:

$$
\mathbb{M}_{k}=\alpha+\beta \zeta_{1}+\gamma \tau_{1} \zeta_{1}+\delta \tau_{1} \zeta_{1} l_{1}
$$

where $\alpha=2 J+J_{\mathrm{ud}} / 2, \beta=-\Gamma \psi_{k}^{(s)}, \gamma=(2 J-\Gamma) \psi_{k}^{(s)}, \delta=J_{\mathrm{ud}} / 2$. In terms of these parameters, the band energies are given by

$$
\begin{aligned}
\chi(i \Omega)= & 4 g^{\prime 2} \sum_{\zeta= \pm} \int_{k} \\
& \times \frac{2\left(\Omega_{\mathbf{k},+}^{(\zeta)}+\Omega_{\mathbf{k},-}^{(\zeta)}\right)\left((\alpha+\beta)\left(\alpha+\beta-4 \gamma \psi^{(s)}\right)+\left(\gamma^{2}-\delta^{2}\right)+\psi^{(s) 2}\left((\alpha+\beta)^{2}+\left(\gamma^{2}-\delta^{2}\right)+\Omega_{\mathbf{k},+}^{(\zeta)} \Omega_{\mathbf{k},-}^{(\zeta)}\right)-\Omega_{\mathbf{k},+}^{(\zeta)} \Omega_{\mathbf{k},-}^{(\zeta)}\right)}{\Omega_{\mathbf{k},+}^{(\zeta)} \Omega_{\mathbf{k},-}^{(\zeta)}\left(\left(\Omega_{\mathbf{k},+}^{(\zeta)}+\Omega_{\mathbf{k},-}^{(\zeta)}\right)^{2}+\Omega^{2}\right)}
\end{aligned}
$$

\section{APPENDIX D: ELIMINATING PHONON MODE}

Here we adiabatically eliminate the phonon mode using the path-integral formulation. If we neglect the phonon dispersion, we can consider the local coupling on each bond, in which case we suppress the bond index and consider a single oscillator.

The relevant Matsubara action is

$$
\begin{aligned}
\mathcal{S}= & \sum_{\omega_{m}} \frac{1}{2} M_{\mathrm{ph}}\left(\omega_{m}^{2}+\Omega_{\mathrm{ph}}^{2}\right) Q_{-\omega_{m}} Q_{\omega_{m}} \\
& +\left[-Z_{\mathrm{ph}} e \omega_{m} A_{\omega_{m}}^{z}+F_{\omega_{m}}\right] Q_{-\omega_{m}} .
\end{aligned}
$$

Here, the Matsubara frequencies are $\omega_{m}=2 \pi m T$ and $Q_{\omega_{m}}$ is the frequency-domain phonon coordinate, with effective mass $M_{\mathrm{ph}}$ and Born effective charge $Z_{\mathrm{eff}} e$. Here $F_{\omega_{m}}$ encodes the spin-system operator the phonon linearly couples to, and $A_{\omega_{n}}^{z}$ is the $z$ component of the electromagnetic vector potential. We work in a gauge where the scalar potential is zero, such that the electric field is purely determined in terms of the vector potential.

Importantly, upon Wick rotation, the dipolar coupling to the electric field becomes $-\omega_{m} A_{\omega_{m}}^{z} Z_{\mathrm{ph}} e Q_{-\omega_{m}}$, and we can identify

$$
\hat{E}_{\omega_{m}}^{z}=\omega_{m} A_{\omega_{m}}^{z} \rightarrow i \omega A^{z}(\omega) \rightarrow-\partial_{t} A^{z}
$$

upon returning to real-frequencies, and then the time-domain.

We can integrate out the phonon mode in the saddlepoint approximation, which is exact for a Gaussian degree of freedom. We then find the effective action describing the electric-field and its coupling to the spin operator. The saddlepoint equation is

$$
M\left(\omega_{m}^{2}+\Omega_{T}^{2}\right) Q_{\omega_{m}}=Z_{\mathrm{ph}} e \omega_{m} A_{\omega_{m}}^{z}-F_{\omega_{m}} .
$$

We then substitute this back in to the action to obtain the effective action for the electric field and magnons of

$$
\begin{aligned}
\mathcal{S}_{\mathrm{eff}}= & \sum_{\omega_{m}}-\frac{1}{2} \frac{1}{M_{\mathrm{ph}}\left(\omega_{m}^{2}+\Omega_{\mathrm{ph}}^{2}\right)}\left(Z_{\mathrm{ph}} e \omega_{m} A_{\omega_{m}}^{z}-F_{\omega_{m}}\right) \\
& \times\left(-Z_{\mathrm{ph}} e \omega_{m} A_{-\omega_{m}}^{z}-F_{-\omega_{m}}\right) .
\end{aligned}
$$

Expanding out these terms, we find the first contribution is purely dressing the dielectric function of the electromagnetic field and is independent of the spin system. We neglect this effect in this paper, though, in principle, it is probably important and should be considered in future works. The last term on the other hand, $\sim F_{\omega_{m}} F_{-\omega_{m}}$ induces an effective spin-spin interaction. Assuming that $F$ is a two-body spin operator, this will then be an emergent four-body term. Again, this is likely to be important and interesting, but because it is independent of the cavity modes we will neglect it in this paper.

Finally, the term of interest is the middle term, which is what leads to the cavity-magnon coupling. We find it to be

$$
\mathcal{S}_{\mathrm{eff}}^{\mathrm{int}}=\sum_{\omega_{m}} \frac{1}{M_{\mathrm{ph}}\left(\omega_{m}^{2}+\Omega_{\mathrm{ph}}^{2}\right)} Z_{\mathrm{ph}} e \omega_{m} A_{\omega_{m}}^{z} F_{-\omega_{m}} .
$$

For low frequencies, $\omega_{m} \ll \Omega_{T}$, we approximate this by a static interaction between the electric field and the magnon operator. This is found by returning to a Hamiltonian, noting that $\omega_{m} A_{\omega_{m}}^{z} \rightarrow \hat{E}^{z}$ and $F_{\omega_{m}} \rightarrow \hat{F}$ in the canonical operator formalism. This yields the desired magnetoelectric interaction Hamiltonian:

$$
H^{\mathrm{int}}=\frac{Z_{\mathrm{ph}} e}{M_{\mathrm{ph}} \Omega_{T}^{2}} \hat{E}^{z} \hat{F} .
$$

In the lattice model, this is then summed over every bond, which is permissible when all interactions are local.

\section{APPENDIX E: CAVITY COUPLING}

Here we present some of the calculations used in modeling the cavity and, in particular, the near-field coupling scheme we outline in the main text. We also outline the approach we take for the calculations on cavity-induced squeezing of magnons.

\section{Near-field cavity}

We first consider the problem of a small metallic sphere with plasma response

$$
\epsilon_{m}(\omega)=\epsilon_{0}\left(1-\Omega_{\mathrm{pl}}^{2} / \omega^{2}\right)
$$

and radius $R$. We consider placing this within the electric field maximum of a terahertz resonator, which we take to have a roughly homogeneous field polarized parallel to the surface of the sample, as illustrated in Fig. 13. Simple electrostatics then yields that the terahertz field $\mathbf{E}_{\text {ext }}(\omega)$ induces a dipole 


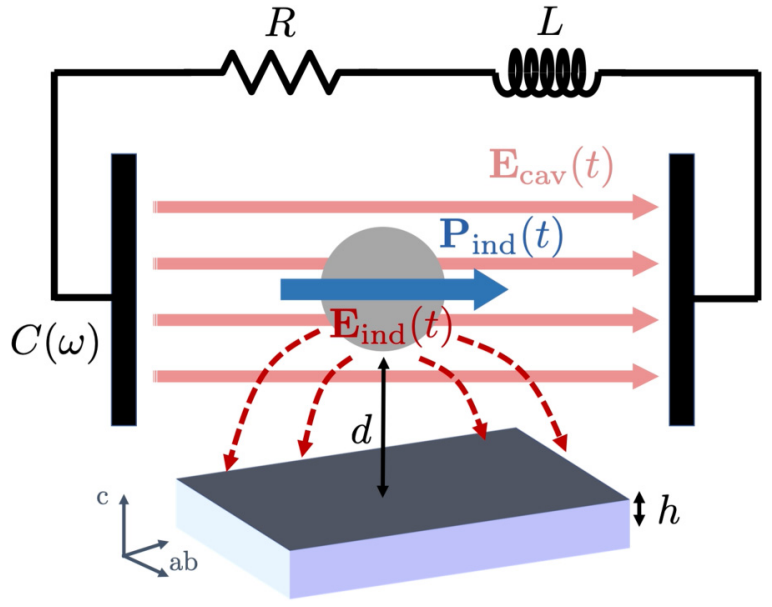

FIG. 13. Schematic of the proposed near-field coupling scheme: A metal object (grey sphere) is placed inside the resonator, which we model as an $R L C$ circuit. The cavity electric field $\mathbf{E}_{\mathrm{cav}}(t)$ induces a dipole moment $\mathbf{P}_{\text {ind }}(t)$ in the metal object. The sample (of thickness $h$ ) is placed a distance $d$ below the induced dipole and experiences a dipole field $\mathbf{E}_{\text {ind }}(t)$, which samples momenta of order $\sim 1 / d$.

moment in the sphere given by

$$
\mathbf{p}(\omega)=4 \pi \epsilon_{0}\left[\frac{\epsilon_{m}(\omega)-\epsilon_{0}}{2 \epsilon_{0}+\epsilon_{m}(\omega)}\right] R^{3} \mathbf{E}_{\mathrm{ext}}(\omega) .
$$

Assuming the plasma frequency is much larger than the resonator frequency, we obtain the simplified result:

$$
\mathbf{p}(\omega)=4 \pi \epsilon_{0} R^{3} \mathbf{E}_{\text {ext }}(\omega) .
$$

We therefore see that the object serves to focus the resonator field to a subwavelength volume, producing a near field which has the same spectral characteristics as the resonator, but with the field profile of a localized electric dipole moment. The induced moment produces the near field

$$
\mathbf{E}_{\text {ind }}=\frac{R^{3}}{r^{3}} \mathbf{E}_{\mathrm{ext}} \cdot[3 \hat{\mathbf{r}} \otimes \hat{\mathbf{r}}-1],
$$

where $\mathbf{r}=r \hat{\mathbf{r}}$ is the vector from the dipole moment to the coordinate.

We next consider the coupling of this moment to the magnons in the sample, which we assume is placed a distance $d$ below the induced dipole, with the $a b$ plane parallel to the induced moment. Our calculations reveal that the magnons couple to the $E_{z}$ component, so we Fourier transform the $E_{z}$ component of the induced field to obtain the dependence on in-plane momentum. We find

$$
E_{z}\left(\omega, \mathbf{q}_{\|}, d\right)=\frac{1}{\sqrt{\text { Area }}} \int d^{2} \mathbf{r} e^{-i \mathbf{r} \cdot \mathbf{q}_{\|}} \frac{-3 R^{3} d}{\left(r^{2}+d^{2}\right)^{\frac{5}{2}}} \mathbf{E}_{\mathrm{ext}} \cdot \hat{\mathbf{r}},
$$

where now we use $\mathbf{r}$ to label the coordinate with respect to the location of the dipole, projected onto the $a b$ plane a distance $d$ below. We can integrate by parts to get

$E_{z}\left(\omega, \mathbf{q}_{\|}, d\right)=\frac{1}{\sqrt{\text { Area }}} d R^{3} i \mathbf{q}_{\|} \cdot \mathbf{E}_{\text {ext }} \int d^{2} \mathbf{r} e^{-i \mathbf{r} \cdot \mathbf{q}_{\|}} \frac{1}{\left(r^{2}+d^{2}\right)^{\frac{3}{2}}}$.
The remaining angular integral is evaluated to be

$$
\int d^{2} \mathbf{r} e^{-i \mathbf{r} \cdot \mathbf{q}_{\|}} \frac{1}{\left(r^{2}+d^{2}\right)^{\frac{3}{2}}}=2 \pi \int_{0}^{\infty} d r \frac{r J_{0}\left(\left|\mathbf{q}_{\|}\right| r\right)}{\left(r^{2}+d^{2}\right)^{\frac{3}{2}}},
$$

and then we evaluate the radial integral, which yields

$$
\int d^{2} \mathbf{r} e^{-i \mathbf{r} \cdot \mathbf{q}_{\|}} \frac{1}{\left(r^{2}+d^{2}\right)^{\frac{3}{2}}}=\frac{2 \pi}{d} e^{-d\left|\mathbf{q}_{\|}\right|} .
$$

We therefore find the momentum space profile:

$$
E_{z}\left(\omega, \mathbf{q}_{\|}, d\right)=\frac{1}{\sqrt{\text { Area }}} R^{3} 2 \pi i \mathbf{q}_{\|} \cdot \mathbf{E}_{\text {ext }} e^{-d\left|\mathbf{q}_{\|}\right|} .
$$

The fact that this decays exponentially is related to the nearfield nature of the profile. We find that this has a maximum in momentum space at $\mathbf{q} \sim 1 / d$, yielding the desired effect of sampling the coupling strength at finite momentum. One assumption we make is that the cavity eigenmode is not changed by the presence of the substrate, which is, in general, not the case. However, we will use this model anyway, bearing in mind the actual parameters in an experiment may be quite different, and more quantitative calculations need to be performed if device geometry is a crucial consideration.

\section{Cavity spectral function}

We now turn our attention to the modeling of $\mathbf{E}_{\text {ext }}$, which is produced by the terahertz resonator. We model the system as an $R L C$ circuit, and model the field as being generated by a parallel-plate capacitor. In that case, the equation of motion for the charge, in the frequency domain, is

$$
\left(-I \omega^{2}-i \omega R+\frac{1}{C_{\text {eff }}(\omega)}\right) Q_{\text {cav }}(\omega)=0,
$$

where $I$ and $R$ are the effective inductance and resistance of the circuit, and $C_{\text {eff }}(\omega)$ is an effective capacitance, which includes the bare geometric capacitance $C_{0}=\epsilon_{0} A_{\text {eff }} / l_{\text {eff }}$, as well as the dressed capacitance due to the embedded object, and its resulting coupling to the magnons below. We make the crude approximation that the field generated by the induced dipole is the same as the vacuum field, which obviously requires refinement in future works. If we consider the sample to have a thickness $h$, we find the electrostatic energy at frequency $\omega$ is determined by the functional

$$
\begin{aligned}
U= & \frac{1}{2} \epsilon_{0} \mathbf{E}_{\mathrm{cav}}^{2} A_{\mathrm{eff}} \ell_{\mathrm{eff}}+\frac{1}{2} \int_{d-h / 2}^{d+h / 2} d z \\
& \times \int_{\mathbf{q}} \chi_{z z}(\omega, \mathbf{q})\left|\mathbf{E}_{\mathrm{cav}} \cdot \boldsymbol{\epsilon}_{\mathbf{q}}(z)\right|^{2},
\end{aligned}
$$

with

$$
\boldsymbol{\epsilon}_{\mathbf{q}}(z)=2 \pi R^{3} i \mathbf{q} e^{-z q} .
$$

We identify the total capacitance as $C_{\text {eff }}=\frac{1}{\ell_{\text {eff }}^{2}} \delta^{2} U / \delta^{2} E$, which gives

$$
C(\omega)=\epsilon_{0} \frac{A_{\mathrm{eff}}}{\ell_{\mathrm{eff}}}+\frac{4 \pi^{2} h R^{6}}{\ell_{\mathrm{eff}}^{2}} \int_{\mathbf{q}} \chi_{z z}(\omega, \mathbf{q})\left|\hat{\mathbf{e}}_{\mathrm{cav}} \cdot \mathbf{q}\right|^{2} \frac{\sinh h q e^{-2 d q}}{h q} .
$$

The second contribution is regarded as the capacitance between the resonator and substrate or, alternatively, is the 
substrate's contribution to the energetic cost of the induced dipole. Therefore, the capacitive coupling to the magnons is given by

$$
C_{\mathrm{mag}}(\omega)=\frac{4 \pi^{2} h R^{6}}{\ell_{\mathrm{eff}}^{2}} \int_{\mathbf{q}} \chi_{z z}(\omega, \mathbf{q})\left|\hat{\mathbf{e}}_{\mathrm{cav}} \cdot \mathbf{q}\right|^{2} \frac{\sinh h q e^{-2 d q}}{h q} .
$$

We therefore end up with the dressed cavity spectral function (up to an overall constant) of

$$
A_{\text {cav }}(\omega)=-\frac{1}{\pi} \mathfrak{s} \frac{1}{\omega^{2}+i \gamma \omega-\Omega_{0}^{2}\left(1+K_{\text {mag }}(\omega)\right)^{-1}},
$$

with the usual relations $\gamma=R / I, \Omega_{0}^{2}=1 /(I C)$ and the dimensionless coupling function

$K_{\mathrm{mag}}(\omega)=\frac{4 \pi^{2} h R^{6}}{\ell_{\mathrm{eff}} A_{\mathrm{eff}} a^{4}} \int_{\mathbf{q}} \frac{\chi_{z z}(\omega, \mathbf{q})}{\epsilon_{0}}\left|\hat{\mathbf{e}}_{\mathrm{cav}} \cdot \mathbf{q}\right|^{2} \frac{\sinh \frac{h}{a}|\mathbf{q}| e^{-2 \frac{d}{a}|\mathbf{q}|}}{\frac{h}{a}|\mathbf{q}|}$.

In the above expression, we have replaced the lattice unit of $a \sim 4 \AA$, originating from the momentum integral, such that now $\mathbf{q}$ is actually dimensionless.

We regard the geometric factors as somewhat phenomenological, in that they are flexible with respect to our model and should be determined from actual device parameters or simulations. For a rough estimate, we take $d / a \sim R / a \sim$ $100, h / a \sim 10$, and $\ell_{\text {eff }} A_{\text {eff }} / a^{3} \sim 10^{6}$. Note this corresponds to $d \sim R \sim 40 \mathrm{~nm}, h \sim 4 \mathrm{~nm}$, and an effective volume of $\ell_{\mathrm{eff}} A_{\mathrm{eff}} \sim 40 \mathrm{~nm} \times 40 \mathrm{~nm} \times 40 \mathrm{~nm}$. If we approximate $\chi$ by $F|\mathbf{q} \cdot \mathbf{N}|^{2} /\left(\Omega^{2}-\omega^{2}\right)$ and consider the case of $\mathbf{N}=\hat{\mathbf{e}}_{\mathrm{cav}}=\mathbf{e}_{x}$, we get a rough estimate for $K$ of

$$
K_{\mathrm{mag}} \sim \frac{3 \cdot 5 !}{8 \cdot 2^{6}} \frac{h(R / d)^{6} a^{2}}{\ell_{\mathrm{eff}} A_{\mathrm{eff}}} \frac{F}{\Omega_{\mathrm{sw}}^{2}-\omega^{2}},
$$

where $F \sim 6.2 \times 10^{4} \mathrm{meV}^{2}$ is the oscillator strength estimated in the main text. The numerical prefactor is of order one, and so the main suppression is due to the disconnect between the capacitor size and the lattice scale, with $h a^{2} / \ell_{\text {eff }} A_{\text {eff }} \sim 10^{-5}$.

Bearing this in mind by approximating

$$
K_{\text {mag }}(\omega)=\alpha^{2} \text {, }
$$

where $\alpha^{2} \sim 10^{-5}$ is the effective coupling constant, we obtain the spectral function

$$
A_{\mathrm{cav}}(\omega)=-\frac{1}{\pi} \Im \frac{1}{\omega^{2}+i \gamma \omega-\Omega_{0}^{2}\left(1+\frac{\alpha^{2} F}{\Omega_{\mathrm{sw}}^{2}-\left(\omega+i 0^{+}\right)^{2}}\right)^{-1}} .
$$

Recall that typical values for $\Omega_{\mathrm{sw}}$ and $F$ are $\Omega_{\mathrm{sw}} \sim 1 \mathrm{THz}$ and $F \sim 3.8 \times 10^{3}(\mathrm{THz})^{2}$.

This can reveal a clear avoided crossing in frequency space as the cavity resonance is tuned through the spin-wave res- onance. This doesn't take dispersion of the spin wave into account, for instance, and this could possibly spoil the effect since dispersion of the resonance will act as a sort of inhomogeneous broadening. Depending on the characteristic momentum of the magnon sampled by the geometry, this may be important.

If we are going to be more careful, we see that the correction due to the dispersion of the mode is approximated as, replacing the lattice unit $a$,

$$
\Omega_{\mathbf{q}}^{2}=\Omega_{0}^{2}+c^{2} \mathbf{q}^{2}
$$

with momentum $\mathbf{q}$ in units of $a^{-1}$ and $c=\sqrt{2} J_{0} a$. Then, we find for the response function $K$, in the limit of $h \ll d$, and including the angular dependence as well,

$$
\begin{aligned}
K_{\mathrm{mag}}(\omega)=5 ! \pi \frac{h a^{2}}{V_{\mathrm{eff}}}\left(\frac{R}{2 d}\right)^{6} & {\left[\frac{3}{4}(\hat{\mathbf{N}} \cdot \hat{\mathbf{E}})^{2}\right.} \\
& \left.+\frac{1}{4}(\hat{\mathbf{N}} \times \hat{\mathbf{E}})^{2}\right] \frac{F}{\Omega_{0}^{2}} g(z, \kappa),
\end{aligned}
$$

with the function

$$
g(z, \kappa)=\frac{1}{5 !} \int_{0}^{\infty} d x x^{5} e^{-x} \frac{1}{1+\kappa^{2} x^{2}-\left(z+i 0^{+}\right)^{2}},
$$

where we have introduced

$$
\begin{aligned}
& z=\left(\omega+i 0^{+}\right) / \Omega_{0}, \\
& \kappa=\frac{c}{2 d \Omega_{0}} .
\end{aligned}
$$

We have also used $\hat{\mathbf{N}}, \hat{\mathbf{E}}$ to indicate the unit vectors along the Néel vector and in-plane cavity polarization, respectively. The previous calculation holds in the limit of $\kappa^{2} \sim \frac{a^{2}}{d^{2}} \frac{J_{0}}{\Gamma} \rightarrow 0$, in which case the function $g$ reduces to the clear resonance at $z=1$. On the other hand, for $\kappa \gtrsim 1$, we cannot discount the dispersion effects, and this will quickly destroy the resonance due to the inhomogeneous broadening of the dispersing magnon. While the ratio $a^{2} / d^{2}$ is clearly quite small (and part of the goal is to enlarge this parameter), the ratio $J_{0} / \Gamma \sim 10^{4}$ is also quite large, reflecting the rapid onset of dispersion. We have inadvertently chosen $d / a \sim 10^{2}$ such that $\kappa \sim 1$. If we decrease $\kappa$ to a value of order .1, we can probably resolve the resonance. This means increasing $d / a$ to be around $d / a \sim 300$, which will in turn decrease the overall size of the coupling constant by $1 / 3^{6}$. This would kill the effect, unless we concomitantly scale $R$ such that $R / d$ remains constant. This means $R \sim d \sim 300 a \sim 1200 \AA$.
[1] S. Choi, J. Choi, R. Landig, G. Kucsko, H. Zhou, J. Isoya, F. Jelezko, S. Onoda, H. Sumiya, V. Khemani, C. v. Keyserlingk, N. Y. Yao, E. Demler, and M. D. Lukin, Observation of discrete time-crystalline order in a disordered dipolar many-body system, Nature (London) 543, 221 (2017).

[2] J. Zhang, P. W. Hess, A. Kyprianidis, P. Becker, A. Lee, J. Smith, G. Pagano, I.-D. Potirniche, A. C. Potter,
A. Vishwanath, N. Y. Yao, and C. Monroe, Observation of a discrete time crystal, Nature (London) 543, 217 (2017).

[3] M. Heyl, Dynamical quantum phase transitions: A review, Rep. Prog. Phys. 81, 054001 (2018).

[4] I.-D. Potirniche, A. C. Potter, M. Schleier-Smith, A. Vishwanath, and N. Y. Yao, Floquet Symmetry-Protected 
Topological Phases in Cold-Atom Systems, Phys. Rev. Lett. 119, 123601 (2017).

[5] A. C. Potter and T. Morimoto, Dynamically enriched topological orders in driven two-dimensional systems, Phys. Rev. B 95, 155126 (2017).

[6] T. Oka and H. Aoki, Photovoltaic Hall effect in graphene, Phys. Rev. B 79, 081406(R) (2009).

[7] N. H. Lindner, G. Refael, and V. Galitski, Floquet topological insulator in semiconductor quantum wells, Nat. Phys. 7, 490 (2011).

[8] T. Kitagawa, E. Berg, M. Rudner, and E. Demler, Topological characterization of periodically driven quantum systems, Phys. Rev. B 82, 235114 (2010).

[9] M. Claassen, H.-C. Jiang, B. Moritz, and T. P. Devereaux, Dynamical time-reversal symmetry breaking and photo-induced chiral spin liquids in frustrated Mott insulators, Nat. Commun. 8, 1192 (2017).

[10] E. V. Boström, M. Claassen, J. W. McIver, G. Jotzu, A. Rubio, and M. A. Sentef, Light-induced topological magnons in twodimensional van der Waals magnets, SciPost Phys. 9, 061 (2020).

[11] D. V. Else, B. Bauer, and C. Nayak, Floquet Time Crystals, Phys. Rev. Lett. 117, 090402 (2016).

[12] H. Bernien, S. Schwartz, A. Keesling, H. Levine, A. Omran, H. Pichler, S. Choi, A. S. Zibrov, M. Endres, M. Greiner, V. Vuletic, and M. D. Lukin, Probing many-body dynamics on a 51-atom quantum simulator, Nature (London) 551, 579 (2017).

[13] F. Mivehvar, H. Ritsch, and F. Piazza, Superradiant Topological Peierls Insulator Inside an Optical Cavity, Phys. Rev. Lett. 118, 073602 (2017).

[14] A. Chiocchetta, D. Kiese, F. Piazza, and S. Diehl, Cavityinduced quantum spin liquids, Nat. Commun. 12, 5901 (2021).

[15] E. Altman and A. Auerbach, Oscillating Superfluidity of Bosons in Optical Lattices, Phys. Rev. Lett. 89, 250404 (2002).

[16] M. Bukov, S. Gopalakrishnan, M. Knap, and E. Demler, Prethermal Floquet Steady States and Instabilities in the Periodically Driven, Weakly Interacting Bose-Hubbard Model, Phys. Rev. Lett. 115, 205301 (2015).

[17] D. N. Basov, R. D. Averitt, and D. Hsieh, Towards properties on demand in quantum materials, Nat. Mater. 16, 1077 (2017).

[18] A. Cavalleri, Photo-induced superconductivity, Contemp. Phys. 59, 31 (2018).

[19] J. Liu, K. Hejazi, and L. Balents, Floquet Engineering of Multiorbital Mott Insulators: Applications to Orthorhombic Titanates, Phys. Rev. Lett. 121, 107201 (2018).

[20] D. M. Kennes, M. Claassen, M. A. Sentef, and C. Karrasch, Light-induced $d$-wave superconductivity through Floquetengineered Fermi surfaces in cuprates, Phys. Rev. B 100, 075115 (2019).

[21] N. Walldorf, D. M. Kennes, J. Paaske, and A. J. Millis, The antiferromagnetic phase of the Floquet-driven Hubbard model, Phys. Rev. B 100, 121110(R) (2019).

[22] M. A. Sentef, A. Tokuno, A. Georges, and C. Kollath, Theory of Laser-Controlled Competing Superconducting and Charge Orders, Phys. Rev. Lett. 118, 087002 (2017).

[23] D. Malz, J. Knolle, and A. Nunnenkamp, Topological magnon amplification, Nat. Commun. 10, 3937 (2019).
[24] M. Gu and J. M. Rondinelli, Nonlinear phononic control and emergent magnetism in Mott insulating titanates, Phys. Rev. B 98, 024102 (2018).

[25] I. Martin, G. Refael, and B. Halperin, Topological Frequency Conversion in Strongly Driven Quantum Systems, Phys. Rev. X 7, 041008 (2017).

[26] A. J. Sternbach, S. H. Chae, S. Latini, A. A. Rikhter, Y. Shao, B. Li, D. Rhodes, B. Kim, P. J. Schuck, X. Xu, X.-Y. Zhu, R. D. Averitt, J. Hone, M. M. Fogler, A. Rubio, and D. N. Basov, Programmable hyperbolic polaritons in van der Waals semiconductors, Science 371, 617 (2021).

[27] T. W. Ebbesen, Hybrid light-matter states in a molecular and material science perspective, Acc. Chem. Res. 49, 2403 (2016).

[28] E. Orgiu, J. George, J. A. Hutchison, E. Devaux, J. F. Dayen, B. Doudin, F. Stellacci, C. Genet, J. Schachenmayer, C. Genes, G. Pupillo, P. Samorì, and T. W. Ebbesen, Conductivity in organic semiconductors hybridized with the vacuum field, Nat. Mater. 14, 1123 (2015).

[29] A. X. Gray, M. C. Hoffmann, J. Jeong, N. P. Aetukuri, D. Zhu, H. Y. Hwang, N. C. Brandt, H. Wen, A. J. Sternbach, S. Bonetti, A. H. Reid, R. Kukreja, C. Graves, T. Wang, P. Granitzka, Z. Chen, D. J. Higley, T. Chase, E. Jal, E. Abreu, M. K. Liu, T. C. Weng, D. Sokaras, D. Nordlund, M. Chollet, R. Alonso-Mori, H. Lemke, J. M. Glownia, M. Trigo, Y. Zhu, H. Ohldag, J. W. Freeland, M. G. Samant, J. Berakdar, R. D. Averitt, K. A. Nelson, S. S. P. Parkin, and H. A. Durr, Ultrafast terahertz field control of electronic and structural interactions in vanadium dioxide, Phys. Rev. B 98, 045104 (2018).

[30] R. Mankowsky, A. Subedi, M. Först, S. O. Mariager, M. Chollet, H. T. Lemke, J. S. Robinson, J. M. Glownia, M. P. Minitti, A. Frano, M. Fechner, N. A. Spaldin, T. Loew, B. Keimer, A. Georges, and A. Cavalleri, Nonlinear lattice dynamics as a basis for enhanced superconductivity in $\mathrm{YBa}_{2} \mathrm{Cu}_{3} \mathrm{O}_{6.5}$, Nature (London) 516, 71 (2014).

[31] S. F. Maehrlein, I. Radu, P. Maldonado, A. Paarmann, M. Gensch, A. M. Kalashnikova, R. V. Pisarev, M. Wolf, P. M. Oppeneer, J. Barker, and T. Kampfrath, Dissecting spinphonon equilibration in ferrimagnetic insulators by ultrafast lattice excitation, Sci. Adv. 4, eaar5164 (2018).

[32] A. S. Disa, M. Fechner, T. F. Nova, B. Liu, M. Först, D. Prabhakaran, P. G. Radaelli, and A. Cavalleri, Polarizing an antiferromagnet by optical engineering of the crystal field, Nat. Phys. 16, 937 (2020).

[33] M. Budden, T. Gebert, M. Buzzi, G. Jotzu, E. Wang, T. Matsuyama, G. Meier, Y. Laplace, D. Pontiroli, M. Riccò, F. Schlawin, D. Jaksch, and A. Cavalleri, Evidence for metastable photo-induced superconductivity in $\mathrm{K} 3 \mathrm{C} 60$, Nat. Phys. 17, 611 (2021).

[34] R. V. Mikhaylovskiy, T. J. Huisman, V. A. Gavrichkov, S. I. Polukeev, S. G. Ovchinnikov, D. Afanasiev, R. V. Pisarev, T. Rasing, and A. V. Kimel, Resonant Pumping of $d$ - $d$ Crystal Field Electronic Transitions as a Mechanism of Ultrafast Optical Control of the Exchange Interactions in Iron Oxides, Phys. Rev. Lett. 125, 157201 (2020).

[35] A. S. McLeod, J. Zhang, M. Q. Gu, F. Jin, G. Zhang, K. W. Post, X. G. Zhao, A. J. Millis, W. B. Wu, J. M. Rondinelli, R. D. Averitt, and D. N. Basov, Multi-messenger nanoprobes of hidden magnetism in a strained manganite, Nat. Mater. 19, 397 (2020). 
[36] D. Afanasiev, J. R. Hortensius, B. A. Ivanov, A. Sasani, E. Bousquet, Y. M. Blanter, R. V. Mikhaylovskiy, A. V. Kimel, and A. D. Caviglia, Ultrafast control of magnetic interactions via light-driven phonons, Nat. Mater. 20, 607 (2021).

[37] H. Qiu, L. Zhou, C. Zhang, J. Wu, Y. Tian, S. Cheng, S. Mi, H. Zhao, Q. Zhang, D. Wu, B. Jin, J. Chen, and P. Wu, Ultrafast spin current generated from an antiferromagnet, Nat. Phys. 17, 388 (2021).

[38] A. Ron, S. Chaudhary, G. Zhang, H. Ning, E. Zoghlin, S. D. Wilson, R. D. Averitt, G. Refael, and D. Hsieh, Ultrafast Enhancement of Ferromagnetic Spin Exchange Induced by Ligand-to-Metal Charge Transfer, Phys. Rev. Lett. 125, 197203 (2020).

[39] M. Buzzi, G. Jotzu, A. Cavalleri, J. I. Cirac, E. A. Demler, B. I. Halperin, M. D. Lukin, T. Shi, Y. Wang, and D. Podolsky, Higgs-Mediated Optical Amplification in a Nonequilibrium Superconductor, Phys. Rev. X 11, 011055 (2021).

[40] A. Kogar, A. Zong, P. E. Dolgirev, X. Shen, J. Straquadine, Y.-Q. Bie, X. Wang, T. Rohwer, I.-C. Tung, Y. Yang, R. Li, J. Yang, S. Weathersby, S. Park, M. E. Kozina, E. J. Sie, H. Wen, P. Jarillo-Herrero, I. R. Fisher, X. Wang et al., Lightinduced charge density wave in $\mathrm{LaTe}_{3}$, Nat. Phys. 16, 159 (2020).

[41] D. J. Lovinger, E. Zoghlin, P. Kissin, G. Ahn, K. Ahadi, P. Kim, M. Poore, S. Stemmer, S. J. Moon, S. D. Wilson, and R. D. Averitt, Magnetoelastic coupling to coherent acoustic phonon modes in the ferrimagnetic insulator $\mathrm{GdTiO}_{3}$, Phys. Rev. B 102, 085138 (2020).

[42] T. F. Nova, A. S. Disa, M. Fechner, and A. Cavalleri, Metastable ferroelectricity in optically strained $\mathrm{SrTiO}_{3}$, Science 364, 1075 (2019).

[43] K. A. Cremin, J. Zhang, C. C. Homes, G. D. Gu, Z. Sun, M. M. Fogler, A. J. Millis, D. N. Basov, and R. D. Averitt, Photoenhanced metastable c-axis electrodynamics in stripe-ordered cuprate $\mathrm{La}_{1.885} \mathrm{Ba}_{0.115} \mathrm{CuO}_{4}$, Proc. Natl. Acad. Sci. USA 116, 19875 (2019).

[44] M. Mitrano, A. Cantaluppi, D. Nicoletti, S. Kaiser, A. Perucchi, S. Lupi, P. Di Pietro, D. Pontiroli, M. Riccó, S. R. Clark, D. Jaksch, and A. Cavalleri, Possible lightinduced superconductivity in $\mathrm{K}_{3} \mathrm{C}_{60}$ at high temperature, Nature (London) 530, 461 (2016).

[45] K. Katsumi, Z. Z. Li, H. Raffy, Y. Gallais, and R. Shimano, Superconducting fluctuations probed by the Higgs mode in $\mathrm{Bi}_{2} \mathrm{Sr}_{2} \mathrm{CaCu}_{2} \mathrm{O}_{8}+\mathrm{x}$ thin films, Phys. Rev. B 102, 054510 (2020).

[46] X. Li, T. Qiu, J. Zhang, E. Baldini, J. Lu, A. M. Rappe, and K. A. Nelson, Terahertz field-induced ferroelectricity in quantum paraelectric SrTiO3, Science 364, 1079 (2019).

[47] X. Shi, W. You, Y. Zhang, Z. Tao, P. M. Oppeneer, X. Wu, R. Thomale, K. Rossnagel, M. Bauer, H. Kapteyn, and M. Murnane, Ultrafast electron calorimetry uncovers a new longlived metastable state in $1 \mathrm{~T}-\mathrm{TaSe}_{2}$ mediated by mode-selective electron-phonon coupling, Sci. Adv. 5, eaav4449 (2019).

[48] R. I. Tobey, D. Prabhakaran, A. T. Boothroyd, and A. Cavalleri, Ultrafast Electronic Phase Transition in $\mathrm{La}_{1 / 2} \mathrm{Sr}_{3 / 2} \mathrm{MnO}_{4}$ by Coherent Vibrational Excitation: Evidence for Nonthermal Melting of Orbital Order, Phys. Rev. Lett. 101, 197404 (2008).

[49] R. Mikhaylovskiy, E. Hendry, A. Secchi, J. Mentink, M. Eckstein, A. Wu, R. Pisarev, V. Kruglyak, M. Katsnelson,
T. Rasing, and A. Kimel, Ultrafast optical modification of exchange interactions in iron oxides, Nat. Commun. 6, 8190 (2015).

[50] A. Zong, A. Kogar, Y.-Q. Bie, T. Rohwer, C. Lee, E. Baldini, E. Ergecen, M. B. Yilmaz, B. Freelon, E. J. Sie, H. Zhou, J. Straquadine, P. Walmsley, P. E. Dolgirev, A. V. Rozhkov, I. R. Fisher, P. Jarillo-Herrero, B. V. Fine, and N. Gedik, Evidence for topological defects in a photoinduced phase transition, Nat. Phys. 15, 27 (2019).

[51] A. Pashkin, M. Porer, M. Beyer, K. W. Kim, A. Dubroka, C. Bernhard, X. Yao, Y. Dagan, R. Hackl, A. Erb, J. Demsar, R. Huber, and A. Leitenstorfer, Femtosecond Response of Quasiparticles and Phonons in Superconducting $\mathrm{YBa}_{2} \mathrm{Cu}_{3} \mathrm{O}_{7-\delta}$ Studied by Wideband Terahertz Spectroscopy, Phys. Rev. Lett. 105, 067001 (2010).

[52] K. Katsumi, N. Tsuji, Y. I. Hamada, R. Matsunaga, J. Schneeloch, R. D. Zhong, G. D. Gu, H. Aoki, Y. Gallais, and R. Shimano, Higgs Mode in the $d$-Wave Superconductor $\mathrm{Bi}_{2} \mathrm{Sr}_{2} \mathrm{CaCu}_{2} \mathrm{O}_{8}+\mathrm{x}$ Driven by an Intense Terahertz Pulse, Phys. Rev. Lett. 120, 117001 (2018).

[53] D. V. Pilon, C. H. Lui, T. H. Han, D. Shrekenhamer, A. J. Frenzel, W. J. Padilla, Y. S. Lee, and N. Gedik, Spin-Induced Optical Conductivity in the Spin-Liquid Candidate Herbertsmithite, Phys. Rev. Lett. 111, 127401 (2013).

[54] H. Niwa, N. Yoshikawa, K. Tomari, R. Matsunaga, D Song, H. Eisaki, and R. Shimano, Light-induced nonequilibrium response of the superconducting cuprate $\mathrm{La}_{2-x} \mathrm{Sr}_{x} \mathrm{CuO}_{4}$, Phys. Rev. B 100, 104507 (2019).

[55] R. Matsunaga and R. Shimano, Nonequilibrium BCS State Dynamics Induced by Intense Terahertz Pulses in a Superconducting NbN Film, Phys. Rev. Lett. 109, 187002 (2012).

[56] E. Baldini, M. A. Sentef, S. Acharya, T. Brumme, E. Sheveleva, F. Lyzwa, E. Pomjakushina, C. Bernhard, M. v. Schilfgaarde, F. Carbone, A. Rubio, and C. Weber, Electronphonon-driven three-dimensional metallicity in an insulating cuprate, Proc. Natl. Acad. Sci. USA 117, 6409 (2020).

[57] P. Sivarajah, A. Steinbacher, B. Dastrup, J. Lu, M. Xiang, W. Ren, S. Kamba, S. Cao, and K. A. Nelson, THz-frequency magnon-phonon-polaritons in the collective strong-coupling regime, J. Appl. Phys. 125, 213103 (2019).

[58] S. Rajasekaran, E. Casandruc, Y. Laplace, D. Nicoletti, G. D. Gu, S. R. Clark, D. Jaksch, and A. Cavalleri, Parametric amplification of a superconducting plasma wave, Nat. Phys. 12, 1012 (2016)

[59] P. E. Dolgirev, A. Zong, M. H. Michael, J. B. Curtis, D. Podolsky, A. Cavalleri, and E. Demler, Periodic dynamics in superconductors induced by an impulsive optical quench, arXiv:2104.07181.

[60] T. Byrnes, N. Y. Kim, and Y. Yamamoto, Exciton-polariton condensates, Nat. Phys. 10, 803 (2014).

[61] M. A. Sentef, M. Ruggenthaler, and A. Rubio, Cavity quantum-electrodynamical polaritonically enhanced electronphonon coupling and its influence on superconductivity, Sci. Adv. 4, eaau6969 (2018).

[62] J. B. Curtis, Z. M. Raines, A. A. Allocca, M. Hafezi, and V. M. Galitski, Cavity Quantum Eliashberg Enhancement of Superconductivity, Phys. Rev. Lett. 122, 167002 (2019).

[63] F. Schlawin, A. Cavalleri, and D. Jaksch, Cavity-Mediated Electron-Photon Superconductivity, Phys. Rev. Lett. 122, 133602 (2019). 
[64] Y. Ashida, A. İmamoğlu, J. Faist, D. Jaksch, A. Cavalleri, and E. Demler, Quantum Electrodynamic Control of Matter: Cavity-Enhanced Ferroelectric Phase Transition, Phys. Rev. X 10, 041027 (2020)

[65] A. A. Allocca, Z. M. Raines, J. B. Curtis, and V. M. Galitski, Cavity superconductor-polaritons, Phys. Rev. B 99, 020504 (2019).

[66] Z. M. Raines, A. A. Allocca, and V. M. Galitski, Manifestations of spin-orbit coupling in a cuprate superconductor, Phys. Rev. B 100, 224512 (2019).

[67] A. Grankin, M. Hafezi, and V. M. Galitski, Enhancement of superconductivity with external phonon squeezing, Phys. Rev. B 104, L220503 (2021).

[68] M. Kiffner, J. R. Coulthard, F. Schlawin, A. Ardavan, and D. Jaksch, Manipulating quantum materials with quantum light, Phys. Rev. B 99, 085116 (2019).

[69] D. N. Basov, A. Asenjo-Garcia, P. J. Schuck, X. Zhu, and A. Rubio, Polariton panorama, Nanophotonics 10, 549 (2020).

[70] M. A. Sentef, J. Li, F. Künzel, and M. Eckstein, Quantum to classical crossover of Floquet engineering in correlated quantum systems, Phys. Rev. Res. 2, 033033 (2020).

[71] M. Kiffner, J. Coulthard, F. Schlawin, A. Ardavan, and D. Jaksch, Mott polaritons in cavity-coupled quantum materials, New J. Phys. 21, 073066 (2019).

[72] H. Dehghani, Z. M. Raines, V. M. Galitski, and M. Hafezi, Optical enhancement of superconductivity via targeted destruction of charge density waves, Phys. Rev. B 101, 224506 (2020).

[73] T. Karzig, C.-E. Bardyn, N. H. Lindner, and G. Refael, Topological Polaritons, Phys. Rev. X 5, 031001 (2015).

[74] T. S. Parvini, V. A. S. V. Bittencourt, and S. V. Kusminskiy, Antiferromagnetic cavity optomagnonics, Phys. Rev. Res. 2, 022027(R) (2020).

[75] Q. Zhang, M. Lou, X. Li, J. L. Reno, W. Pan, J. D. Watson, M. J. Manfra, and J. Kono, Collective non-perturbative coupling of 2D electrons with high-quality-factor terahertz cavity photons, Nat. Phys. 12, 1005 (2016).

[76] G. Mazza and A. Georges, Superradiant Quantum Materials, Phys. Rev. Lett. 122, 017401 (2019).

[77] G. Scalari, C. Maissen, D. Turčinková, D. Hagenmüller, S. D. Liberato, C. Ciuti, C. Reichl, D. Schuh, W. Wegscheider, M. Beck, and J. Faist, Ultrastrong coupling of the cyclotron transition of a $2 \mathrm{D}$ electron gas to a $\mathrm{THz}$ metamaterial, Science $\mathbf{3 3 5}$, 1323 (2012).

[78] M. Geiser, F. Castellano, G. Scalari, M. Beck, L. Nevou, and J. Faist, Ultrastrong Coupling Regime and Plasmon Polaritons in Parabolic Semiconductor Quantum Wells, Phys. Rev. Lett. 108, 106402 (2012).

[79] A. Thomas, E. Devaux, K. Nagarajan, T. Chervy, M. Seidel, D. Hagenmuller, S. Schutz, J. Schachenmayer, C. Genet, G. Pupillo, and T. W. Ebbesen, Exploring superconductivity under strong coupling with the vacuum electromagnetic field, arXiv: 1911.01459

[80] Z. M. Raines, A. A. Allocca, M. Hafezi, and V. M. Galitski, Cavity Higgs polaritons, Phys. Rev. Res. 2, 013143 (2020).

[81] J. Schachenmayer, C. Genes, E. Tignone, and G. Pupillo, Cavity-Enhanced Transport of Excitons, Phys. Rev. Lett. 114, 196403 (2015).
[82] D. Hagenmller, J. Schachenmayer, S. Schtz, C. Genes, and G. Pupillo, Cavity-Enhanced Transport of Charge, Phys. Rev. Lett. 119, 223601 (2017).

[83] Y. Ashida, A. Imamoglu, and E. Demler, Nonperturbative waveguide quantum electrodynamics, arXiv:2105.08833.

[84] K. Head-Marsden, J. Flick, C. J. Ciccarino, and P. Narang, Quantum information and algorithms for correlated quantum matter, Chem. Rev. 121, 3061 (2021)

[85] N. Rivera, J. Flick, and P. Narang, Variational Theory of Nonrelativistic Quantum Electrodynamics, Phys. Rev. Lett. 122, 193603 (2019).

[86] G. R. Keiser, N. Karl, S. R. U. Haque, I. Brener, D. M. Mittleman, and R. D. Averitt, Structural tuning of nonlinear terahertz metamaterials using broadside coupled split ring resonators, AIP Adv. 11, 095103 (2021).

[87] E. Dagotto, Correlated electrons in high-temperature superconductors, Rev. Mod. Phys. 66, 763 (1994).

[88] M. Imada, A. Fujimori, and Y. Tokura, Metal-insulator transitions, Rev. Mod. Phys. 70, 1039 (1998).

[89] P. Curty and H. Beck, Thermodynamics and Phase Diagram of High Temperature Superconductors, Phys. Rev. Lett. 91, 257002 (2003).

[90] S. Peli, S. D. Conte, R. Comin, N. Nembrini, A. Ronchi, P. Abrami, F. Banfi, G. Ferrini, D. Brida, S. Lupi, M. Fabrizio, A. Damascelli, M. Capone, G. Cerullo, and C. Giannetti, Mottness at finite doping and charge instabilities in cuprates, Nat. Phys. 13, 806 (2017).

[91] B. Keimer, S. A. Kivelson, M. R. Norman, S. Uchida, and J. Zaanen, From quantum matter to high-temperature superconductivity in copper oxides, Nature (London) 518, 179 (2015).

[92] Z. M. Raines, V. G. Stanev, and V. M. Galitski, Enhancement of superconductivity via periodic modulation in a threedimensional model of cuprates, Phys. Rev. B 91, 184506 (2015).

[93] M. Fechner and N. A. Spaldin, Effects of intense optical phonon pumping on the structure and electronic properties of yttrium barium copper oxide, Phys. Rev. B 94, 134307 (2016).

[94] Y. Wang, C.-C. Chen, B. Moritz, and T. P. Devereaux, LightEnhanced Spin Fluctuations and d-Wave Superconductivity at a Phase Boundary, Phys. Rev. Lett. 120, 246402 (2018).

[95] J.-i. Okamoto, A. Cavalleri, and L. Mathey, Theory of Enhanced Interlayer Tunneling in Optically Driven High-Tc Superconductors, Phys. Rev. Lett. 117, 227001 (2016).

[96] D. Hagenmller, J. Schachenmayer, C. Genet, T. W. Ebbesen, and G. Pupillo, Enhancement of the electron-phonon scattering induced by intrinsic surface plasmon-phonon polaritons, ACS Photon. 6, 1073 (2019).

[97] M. Woerner, C. Somma, K. Reimann, T. Elsaesser, P. Q. Liu, Y. Yang, J. L. Reno, and I. Brener, Terahertz Driven Amplification of Coherent Optical Phonons in GaAs Coupled to a Metasurface, Phys. Rev. Lett. 122, 107402 (2019).

[98] H. Sumikura, T. Wang, P. Li, A.-K. U. Michel, A. Heßler, L. Jung, M. Lewin, M. Wuttig, D. N. Chigrin, and T. Taubner, Highly confined and switchable mid-infrared surface phonon polariton resonances of planar circular cavities with a phase change material, Nano Lett. 19, 2549 (2019).

[99] Q. Zhang, Z. Zhen, Y. Yang, G. Gan, D. Jariwala, and X. Cui, Hybrid phonon-polaritons at atomically-thin van der Waals heterointerfaces for infrared optical modulation, Opt. Express 27, 18585 (2019). 
[100] A. J. Giles, S. Dai, I. Vurgaftman, T. Hoffman, S. Liu, L. Lindsay, C. T. Ellis, N. Assefa, I. Chatzakis, T. L. Reinecke, J. G. Tischler, M. M. Fogler, J. H. Edgar, D. N. Basov, and J. D. Caldwell, Ultralow-loss polaritons in isotopically pure boron nitride, Nat. Mater. 17, 134 (2018).

[101] T. Terashige, T. Ono, T. Miyamoto, T. Morimoto, H. Yamakawa, N. Kida, T. Ito, T. Sasagawa, T. Tohyama, and H. Okamoto, Doublon-holon pairing mechanism via exchange interaction in two-dimensional cuprate Mott insulators, Sci. Adv. 5, eaav2187 (2019).

[102] V. J. Emery, Theory of High-Tc Superconductivity in Oxides, Phys. Rev. Lett. 58, 2794 (1987).

[103] J. R. Schrieffer, X. G. Wen, and S. C. Zhang, Dynamic spin fluctuations and the bag mechanism of high-Tc superconductivity, Phys. Rev. B 39, 11663 (1989).

[104] C. L. Kane, P. A. Lee, and N. Read, Motion of a single hole in a quantum antiferromagnet, Phys. Rev. B 39, 6880 (1989).

[105] A. H. MacDonald, S. M. Girvin, and D. Yoshioka, $\frac{t}{U}$ expansion for the Hubbard model, Phys. Rev. B 37, 9753 (1988).

[106] K. A. Chao, J. Spałek, and A. M. Oleś, Canonical perturbation expansion of the Hubbard model, Phys. Rev. B 18, 3453 (1978).

[107] D. J. Scalapino, E. Loh, and J. E. Hirsch, d-wave pairing near a spin-density-wave instability, Phys. Rev. B 34, 8190 (1986).

[108] A. M. Oleś, Comment on " $t / U$ expansion for the Hubbard model," Phys. Rev. B 41, 2562 (1990).

[109] J. P. Carbotte, E. Schachinger, and D. N. Basov, Coupling strength of charge carriers to spin fluctuations in high-temperature superconductors, Nature (London) 401, 354 (1999).

[110] A. Abanov, A. V. Chubukov, and J. Schmalian, Quantumcritical theory of the spin-fermion model and its application to cuprates: Normal state analysis, Adv. Phys. 52, 119 (2003).

[111] P. A. Lee, N. Nagaosa, and X.-G. Wen, Doping a Mott insulator: Physics of high-temperature superconductivity, Rev. Mod. Phys. 78, 17 (2006).

[112] S. Sachdev, Quantum criticality and the phase diagram of the cuprates, Physica C: Supercond. Applic. 470, S4 (2010).

[113] F. Grusdt, M. Kánasz-Nagy, A. Bohrdt, C. S. Chiu, G. Ji, M. Greiner, D. Greif, and E. Demler, Parton Theory of Magnetic Polarons: Mesonic Resonances and Signatures in Dynamics, Phys. Rev. X 8, 011046 (2018).

[114] T. Sakai, D. Poilblanc, and D. J. Scalapino, Hole pairing and phonon dynamics in generalized two-dimensional t-J Holstein models, Phys. Rev. B 55, 8445 (1997)

[115] A. S. Mishchenko, N. Nagaosa, Z.-X. Shen, G. De Filippis, V. Cataudella, T. P. Devereaux, C. Bernhard, K. W. Kim, and J. Zaanen, Charge Dynamics of Doped Holes in High $T_{\mathrm{c}}$ Cuprate Superconductors: A Clue from Optical Conductivity, Phys. Rev. Lett. 100, 166401 (2008).

[116] S. Sachdev, E. Berg, S. Chatterjee, and Y. Schattner, Spin density wave order, topological order, and Fermi surface reconstruction, Phys. Rev. B 94, 115147 (2016).

[117] P. A. Lee and N. Nagaosa, Collective modes in the superconducting ground states in the gauge theory description of the cuprates, Phys. Rev. B 68, 024516 (2003).
[118] F. C. Zhang and T. M. Rice, Effective Hamiltonian for the superconducting Cu oxides, Phys. Rev. B 37, 3759 (1988).

[119] P. A. Fleury and R. Loudon, Scattering of light by one- and two-magnon excitations, Phys. Rev. 166, 514 (1968).

[120] A. V. Chubukov and D. M. Frenkel, Resonant Two-Magnon Raman Scattering in Antiferromagnetic Insulators, Phys. Rev. Lett. 74, 3057 (1995).

[121] J. Zhao, A. V. Bragas, D. J. Lockwood, and R. Merlin, Magnon Squeezing in an Antiferromagnet: Reducing the Spin Noise Below the Standard Quantum Limit, Phys. Rev. Lett. 93, 107203 (2004).

[122] T. P. Devereaux, A. Virosztek, and A. Zawadowski, Chargetransfer fluctuation, d-wave superconductivity, and the $B_{1 g}$ Raman phonon in cuprates, Phys. Rev. B 51, 505 (1995).

[123] T. P. Devereaux, A. Virosztek, and A. Zawadowski, Neutron scattering and the $B_{1 g}$ phonon in the cuprates, Phys. Rev. B 59, 14618 (1999).

[124] Y. Wang, T. P. Devereaux, and C.-C. Chen, Theory of timeresolved Raman scattering in correlated systems: Ultrafast engineering of spin dynamics and detection of thermalization, Phys. Rev. B 98, 245106 (2018).

[125] J. Lee and B. Min, Phonon-induced magnon Raman scattering in high Tc cuprates, Solid State Commun. 99, 627 (1996).

[126] T. T. Mai, K. F. Garrity, A. McCreary, J. Argo, J. R. Simpson, V. Doan-Nguyen, R. Valdes Aguilar, and A. High Walker, Magnon-phonon hybridization in quasi-2D antiferromagnet $\mathrm{MnPSe}_{3}$, arXiv:2011.12557.

[127] U. F. P. Seifert and L. Balents, Optical excitation of magnons in an easy-plane antiferromagnet: Application to $\mathrm{Sr}_{2} \mathrm{IrO}_{4}$, Phys. Rev. B 100, 125161 (2019).

[128] A. Bolens, Theory of electronic magnetoelectric coupling in d5 Mott insulators, Phys. Rev. B 98, 125135 (2018).

[129] K. Grishunin, E. A. Mashkovich, A. M. Balbashov, A. K. Zvezdin, and A. V. Kimel, Excitation and detection of $\mathrm{THz}$ coherent spin waves in antiferromagnetic $\alpha-\mathrm{Fe}_{2} \mathrm{O}_{3}$, Phys. Rev. B 104, 024419 (2021)

[130] T. Kampfrath, A. Sell, G. Klatt, A. Pashkin, S. Mährlein, T. Dekorsy, M. Wolf, M. Fiebig, A. Leitenstorfer, and R. Huber, Coherent terahertz control of antiferromagnetic spin waves, Nat. Photon. 5, 31 (2011).

[131] K. Yamaguchi, M. Nakajima, and T. Suemoto, Coherent Control of Spin Precession Motion with Impulsive Magnetic Fields of Half-Cycle Terahertz Radiation, Phys. Rev. Lett. 105, 237201 (2010).

[132] A. H. M. Reid, T. Rasing, R. V. Pisarev, H. A. Dürr, and M. C. Hoffmann, Terahertz-driven magnetism dynamics in the orthoferrite $\mathrm{DyFeO}_{3}$, Appl. Phys. Lett. 106, 082403 (2015).

[133] J. Nishitani, K. Kozuki, T. Nagashima, and M. Hangyo, Terahertz radiation from coherent antiferromagnetic magnons excited by femtosecond laser pulses, Appl. Phys. Lett. 96, 221906 (2010).

[134] J. Xu, C. Zhong, X. Han, D. Jin, L. Jiang, and X. Zhang, Floquet Cavity Electromagnonics, Phys. Rev. Lett. 125, 237201 (2020).

[135] X. Zhang, C.-L. Zou, L. Jiang, and H. X. Tang, Strongly Coupled Magnons and Cavity Microwave Photons, Phys. Rev. Lett. 113, 156401 (2014).

[136] J. Li, S.-Y. Zhu, and G. S. Agarwal, Squeezed states of magnons and phonons in cavity magnomechanics, Phys. Rev. A 99, 021801(R) (2019). 
[137] T. Neuman, D. S. Wang, and P. Narang, Nanomagnonic Cavities for Strong Spin-Magnon Coupling and Magnon-Mediated Spin-Spin Interactions, Phys. Rev. Lett. 125, 247702 (2020).

[138] B. Keimer, R. J. Birgeneau, A. Cassanho, Y. Endoh, M. Greven, M. A. Kastner, and G. Shirane, Soft phonon behavior and magnetism at the low temperature structural phase transition of $\mathrm{La}_{1.65} \mathrm{Nd}_{0.35} \mathrm{CuO}_{4}$, Z. Phys. B 91, 373 (1993).

[139] T. J. Yen, W. J. Padilla, N. Fang, D. C. Vier, D. R. Smith, J. B. Pendry, D. N. Basov, and X. Zhang, Terahertz magnetic response from artificial materials, Science 303, 1494 (2004).

[140] I.-C. Benea-Chelmus, Y. Salamin, F. F. Settembrini, Y. Fedoryshyn, W. Heni, D. L. Elder, L. R. Dalton, J. Leuthold, and J. Faist, Electro-optic interface for ultrasensitive intracavity electric field measurements at microwave and terahertz frequencies, Optica 7, 498 (2020).

[141] J. Zhang, X. Chen, S. Mills, T. Ciavatti, Z. Yao, R. Mescall, H. Hu, V. Semenenko, Z. Fei, H. Li, V. Perebeinos, H. Tao, Q. Dai, X. Du, and M. Liu, Terahertz nanoimaging of graphene, ACS Photon. 5, 2645 (2018).

[142] S. Klingler, H. Maier-Flaig, R. Gross, C.-M. Hu, H. Huebl, S. T. B. Goennenwein, and M. Weiler, Combined Brillouin light scattering and microwave absorption study of magnonphoton coupling in a split-ring resonator/YIG film system, Appl. Phys. Lett. 109, 072402 (2016).

[143] S. Mandal, L. N. Kapoor, S. Ghosh, J. Jesudasan, S. Manni, A. Thamizhavel, P. Raychaudhuri, V. Singh, and M. M. Deshmukh, Coplanar Cavity for Strong Coupling Between Photons and Magnons in van der Waals Antiferromagnet, Appl. Phys. Lett. 117, 263101 (2020).

[144] B.-Y. Jiang, L. M. Zhang, A. H. C. Neto, D. N. Basov, and M. M. Fogler, Generalized spectral method for near-field optical microscopy, J. Appl. Phys. 119, 054305 (2016).

[145] Y. Wu, Metastructure-enhanced terahertz magnon-polaritons, $\mathrm{Ph} . D$. thesis, University of California, Los Angeles, 2020.

[146] J. S. Schalch, K. Post, G. Duan, X. Zhao, Y. D. Kim, J. Hone, M. M. Fogler, X. Zhang, D. N. Basov, and R. D. Averitt, Strong metasurface-Josephson plasma resonance coupling in superconducting $\mathrm{La}_{2-x} \mathrm{Sr}_{x} \mathrm{CuO}_{4}$, Adv. Opt. Mater. 7, 1900712 (2019).

[147] J. Sloan, N. Rivera, and J. D. Joannopoulos, I. Kaminer, M. Soljačić, Controlling spins with surface magnon polaritons, Phys. Rev. B 100, 235453 (2019).

[148] C. Maissen, G. Scalari, F. Valmorra, M. Beck, J. Faist, S. Cibella, R. Leoni, C. Reichl, C. Charpentier, and W. Wegscheider, Ultrastrong coupling in the near field of complementary split-ring resonators, Phys. Rev. B 90, 205309 (2014).

[149] D. MacNeill, J. T. Hou, D. R. Klein, P. Zhang, P. JarilloHerrero, and L. Liu, Gigahertz Frequency Antiferromagnetic Resonance and Strong Magnon-Magnon Coupling in the Layered Crystal $\mathrm{CrCl}_{3}$, Phys. Rev. Lett. 123, 047204 (2019).

[150] A. Bayer, M. Pozimski, S. Schambeck, D. Schuh, R. Huber, D. Bougeard, and C. Lange, Terahertz light-matter interaction beyond unity coupling strength, Nano Lett. 17, 6340 (2017).

[151] G. L. Paravicini-Bagliani, F. Appugliese, E. Richter, F. Valmorra, J. Keller, M. Beck, N. Bartolo, C. Rössler, T. Ihn, K. Ensslin, C. Ciuti, G. Scalari, and J. Faist, Magneto-transport controlled by Landau polariton states, Nat. Phys. 15, 186 (2019).
[152] D. Coffey, T. M. Rice, and F. C. Zhang, DzyaloshinskiiMoriya interaction in the cuprates, Phys. Rev. B 44, 10112 (1991).

[153] N. E. Bonesteel, Theory of anisotropic superexchange in insulating cuprates, Phys. Rev. B 47, 11302 (1993).

[154] L. Shekhtman, O. Entin-Wohlman, and A. Aharony, Moriya's Anisotropic Superexchange Interaction, Frustration, and Dzyaloshinsky's Weak Ferromagnetism, Phys. Rev. Lett. 69, 836 (1992).

[155] W. Koshibae, Y. Ohta, and S. Maekawa, Comment on "Moriya's Anisotropic Superexchange Interaction, Frustration, and Dzyaloshinsky's Weak Ferromagnetism,” Phys. Rev. Lett. 71, 467 (1993).

[156] L. Shekhtman, O. Entin-Wohlman, and A. Aharony, Shekhtman, Entin-Wohlman, and Aharony Reply, Phys. Rev. Lett. 71, 468 (1993).

[157] W. Koshibae, Y. Ohta, and S. Maekawa, Electronic and magnetic structures of cuprates with spin-orbit interaction, Phys. Rev. B 47, 3391 (1993).

[158] C. Wu, J. Zaanen, and S.-C. Zhang, Spin-Orbit CouplingInduced Magnetic Phase in the $d$-Density-Wave Phase of $\mathrm{La}_{2-x} \mathrm{Ba}_{x} \mathrm{CuO}_{4}$ Superconductors, Phys. Rev. Lett. 95, 247007 (2005).

[159] W. A. Atkinson, Microscopic model for the hidden Rashba effect in $\mathrm{YBa}_{2} \mathrm{Cu}_{3} \mathrm{O}_{6+x}$, Phys. Rev. B 101, 024513 (2020).

[160] K. Cao, F. Giustino, and P. G. Radaelli, Theory of Electromagnons in CuO, Phys. Rev. Lett. 114, 197201 (2015).

[161] D. M. Juraschek, M. Fechner, A. V. Balatsky, and N. A. Spaldin, Dynamical multiferroicity, Phys. Rev. Mater. 1, 014401 (2017).

[162] D. Talbayev, S. A. Trugman, S. Lee, H. T. Yi, S.-W. Cheong, and A. J. Taylor, Long-wavelength magnetic and magnetoelectric excitations in the ferroelectric antiferromagnet $\mathrm{BiFeO}_{3}$, Phys. Rev. B 83, 094403 (2011).

[163] H. Katsura, A. V. Balatsky, and N. Nagaosa, Dynamical Magnetoelectric Coupling in Helical Magnets, Phys. Rev. Lett. 98, 027203 (2007).

[164] A. M. Shuvaev, V. D. Travkin, V. Y. Ivanov, A. A. Mukhin, and A. Pimenov, Evidence for Electroactive Excitation of the Spin Cycloid in $\mathrm{TbMnO}_{3}$, Phys. Rev. Lett. 104, 097202 (2010).

[165] V. I. Fal'ko and B. N. Narozhny, Triplet pairing due to spin-orbit-assisted electron-phonon coupling, Phys. Rev. B 74, 012501 (2006).

[166] M. Lee, H.-J. Lee, J. H. Lee, and S. B. Chung, Topological superconductivity from transverse optical phonons in oxide heterostructures, Phys. Rev. Mater. 4, 034202 (2020).

[167] S. Chaudhary, A. Haim, Y. Peng, and G. Refael, Phononinduced Floquet topological phases protected by space-time symmetries, Phys. Rev. Res. 2, 043431 (2020).

[168] B. Ma and G. Fiete, Dzyaloshinskii-moriya induced topological magnon-phonon hybridization in 2D antiferromagnetic insulators with tunable Chern numbers, arXiv:2107.11484.

[169] L. Zhao, C. A. Belvin, R. Liang, D. A. Bonn, W. N. Hardy, N. P. Armitage, and D. Hsieh, A global inversion-symmetrybroken phase inside the pseudogap region of $\mathrm{YBa}_{2} \mathrm{Cu}_{3} \mathrm{O}_{y}$, Nat. Phys. 13, 250 (2017). 
[170] Z. Sun, M. M. Fogler, D. N. Basov, and A. J. Millis, Collective modes and terahertz near-field response of superconductors, Phys. Rev. Res. 2, 023413 (2020).

[171] A. Bitzer, H. Merbold, A. Thoman, T. Feurer, H. Helm, and M. Walther, Terahertz near-field imaging of electric and magnetic resonances of a planar metamaterial, Opt. Express 17, 3826 (2009).

[172] Q. Lu, A. T. Bollinger, X. He, R. Sundling, I. Bozovic, and A. Gozar, Surface Josephson plasma waves in a high-temperature superconductor, npj Quantum Mater. 5, 69 (2020).

[173] J. Zhang, Infrared and Terahertz Near-Field Spectroscopy and Microscopy on 3d and 4d Correlated Electron Materials, Ph.D. thesis, State University of New York at Stony Brook, 2019.

[174] D. N. Basov, M. M. Fogler, and F. J. García de Abajo, Polaritons in van der Waals materials, Science 354, aag1992 (2016).

[175] H. T. Stinson, J. S. Wu, B. Y. Jiang, Z. Fei, A. S. Rodin, B. C. Chapler, A. S. McLeod, A. Castro Neto, Y. S. Lee, M. M. Fogler, and D. N. Basov, Infrared nanospectroscopy and imaging of collective superfluid excitations in anisotropic superconductors, Phys. Rev. B 90, 014502 (2014).

[176] Z. Fei, G. O. Andreev, W. Bao, L. M. Zhang, A. S. McLeod, C. Wang, M. K. Stewart, Z. Zhao, G. Dominguez, M. Thiemens, M. M. Fogler, M. J. Tauber, A. H. Castro-Neto, C. N. Lau, F. Keilmann, and D. N. Basov, Infrared nanoscopy of dirac plasmons at the graphene- $\mathrm{SiO}_{2}$ interface, Nano Lett. 11, 4701 (2011).

[177] S. S. Sunku, G. X. Ni, B. Y. Jiang, H. Yoo, A. Sternbach, A. S. McLeod, T. Stauber, L. Xiong, T. Taniguchi, K. Watanabe, P. Kim, M. M. Fogler, and D. N. Basov, Photonic crystals for nano-light in moiré graphene superlattices, Science 362, 1153 (2018).

[178] B. Normand, H. Kohno, and H. Fukuyama, Spin-phonon coupling in the single-layer extended t-J model, Phys. Rev. B 53, 856 (1996).

[179] E. Erlandsen, A. Kamra, A. Brataas, and A. Sudbø, Enhancement of superconductivity mediated by antiferromagnetic squeezed magnons, Phys. Rev. B 100, 100503(R) (2019).

[180] E. Erlandsen and A. Sudbø, Schwinger boson study of superconductivity mediated by antiferromagnetic spin fluctuations, Phys. Rev. B 102, 214502 (2020).

[181] D. M. Kennes, E. Y. Wilner, D. R. Reichman, and A. J. Millis, Transient superconductivity from electronic squeezing of optically pumped phonons, Nat. Phys. 13, 479 (2017).

[182] M. Babadi, M. Knap, I. Martin, G. Refael, and E. Demler, Theory of parametrically amplified electron-phonon superconductivity, Phys. Rev. B 96, 014512 (2017).

[183] M. Knap, M. Babadi, G. Refael, I. Martin, and E. Demler, Dynamical Cooper pairing in nonequilibrium electron-phonon systems, Phys. Rev. B 94, 214504 (2016).

[184] Y. Murakami, N. Tsuji, M. Eckstein, and P. Werner, Nonequilibrium steady states and transient dynamics of conventional superconductors under phonon driving, Phys. Rev. B 96, 045125 (2017).

[185] This is aside from the possible reports of inversion symmetry breaking [169,273,274], which we do not address here.

[186] P. W. Anderson, New approach to the theory of superexchange interactions, Phys. Rev. 115, 2 (1959).

[187] I. Dzyaloshinsky, A thermodynamic theory of "weak" ferromagnetism of antiferromagnetics, J. Phys. Chem. Solids 4, 241 (1958).
[188] T. Moriya, Anisotropic superexchange interaction and weak ferromagnetism, Phys. Rev. 120, 91 (1960).

[189] T. Das and A. V. Balatsky, Engineering three-dimensional topological insulators in Rashba-type spin-orbit coupled heterostructures, Nat. Commun. 4, 1972 (2013).

[190] N. Harrison, B. J. Ramshaw, and A. Shekhter, Nodal bilayersplitting controlled by spin-orbit interactions in underdoped high-Tc cuprates, Sci. Rep. 5, 10914 (2015).

[191] K. Gotlieb, C.-Y. Lin, M. Serbyn, W. Zhang, C. L. Smallwood, C. Jozwiak, H. Eisaki, Z. Hussain, A. Vishwanath, and A. Lanzara, Revealing hidden spin-momentum locking in a high-temperature cuprate superconductor, Science 362, 1271 (2018).

[192] A. C. Potter, T. Senthil, and P. A. Lee, Mechanisms for subgap optical conductivity in Herbertsmithite, Phys. Rev. B 87, 245106 (2013).

[193] J. Son, B. C. Park, C. H. Kim, H. Cho, S. Y. Kim, L. J. Sandilands, C. Sohn, J.-G. Park, S. J. Moon, and T. W. Noh, Unconventional spin-phonon coupling via the DzyaloshinskiiMoriya interaction, npj Quantum Mater. 4, 17 (2019).

[194] P. Padmanabhan, F. L. Buessen, R. Tutchton, K. W. C. Kwock, S. Gilinsky, M. C. Lee, M. A. McGuire, S. R. Singamaneni, D. A. Yarotski, A. Paramekanti, J. X. Zhu, and R. P. Prasankumar, Coherent helicity-dependent spin-phonon oscillations in the ferromagnetic van der Waals crystal CrI3, arXiv:2010.04915.

[195] D. M. Juraschek, Q. N. Meier, and P. Narang, Parametric Excitation of an Optically Silent Goldstone-Like Phonon Mode, Phys. Rev. Lett. 124, 117401 (2020).

[196] D. M. Juraschek, P. Narang, and N. A. Spaldin, Phonomagnetic analogs to opto-magnetic effects, Phys. Rev. Res. 2, 043035 (2020).

[197] W. Stephan, M. Capone, M. Grilli, and C. Castellani, Influence of electron-phonon interaction on superexchange, Phys. Lett. A 227, 120 (1997).

[198] Y. Mukai, H. Hirori, T. Yamamoto, H. Kageyama, and K. Tanaka, Antiferromagnetic resonance excitation by terahertz magnetic field resonantly enhanced with split ring resonator, Appl. Phys. Lett. 105, 022410 (2014).

[199] H. Y. Yuan, S. Zheng, Q. He, J. Xiao, and R. A. Duine, Unconventional magnon excitation by off-resonant microwaves, Phys. Rev. B 103, 134409 (2021).

[200] H. Y. Yuan and X. R. Wang, Magnon-photon coupling in antiferromagnets, Appl. Phys. Lett. 110, 082403 (2017).

[201] R. E. Camley, Long-Wavelength Surface Spin Waves on Antiferromagnets, Phys. Rev. Lett. 45, 283 (1980).

[202] J. Liu, L. Wang, and K. Shen, Dipolar spin waves in uniaxial easy-axis antiferromagnets: A natural topological nodal-line semimetal, Phys. Rev. Res. 2, 023282 (2020).

[203] Y. Tanabe, T. Moriya, and S. Sugano, Magnon-Induced Electric Dipole Transition Moment, Phys. Rev. Lett. 15, 1023 (1965).

[204] T. Moriya, Theory of absorption and scattering of light by magnetic crystals, J. Appl. Phys. 39, 1042 (1968).

[205] B. S. Shastry and B. I. Shraiman, Theory of Raman Scattering in Mott-Hubbard Systems, Phys. Rev. Lett. 65, 1068 (1990).

[206] H. Katsura, N. Nagaosa, and A. V. Balatsky, Spin Current and Magnetoelectric Effect in Noncollinear Magnets, Phys. Rev. Lett. 95, 057205 (2005) 
[207] C. Jia, S. Onoda, N. Nagaosa, and J. H. Han, Bond electronic polarization induced by spin, Phys. Rev. B 74, 224444 (2006).

[208] C. Jia, S. Onoda, N. Nagaosa, and J. H. Han, Microscopic theory of spin-polarization coupling in multiferroic transition metal oxides, Phys. Rev. B 76, 144424 (2007).

[209] L. N. Bulaevskii, C. D. Batista, M. V. Mostovoy, and D. I. Khomskii, Electronic orbital currents and polarization in Mott insulators, Phys. Rev. B 78, 024402 (2008).

[210] D. M. Juraschek, D. S. Wang, and P. Narang, Sum-frequency excitation of coherent magnons, Phys. Rev. B 103, 094407 (2021).

[211] M. Opel, R. Hackl, T. P. Devereaux, A. Virosztek, A. Zawadowski, A. Erb, E. Walker, H. Berger, and L. Forró, Physical origin of the buckling in $\mathrm{CuO} 2$ : Electron-phonon coupling and Raman spectra, Phys. Rev. B 60, 9836 (1999).

[212] B. Liu, M. Först, M. Fechner, D. Nicoletti, J. Porras, T. Loew, B. Keimer, and A. Cavalleri, Pump Frequency Resonances for Light-Induced Incipient Superconductivity in $\mathrm{YBa}_{2} \mathrm{Cu}_{3} \mathrm{O}_{6.5}$, Phys. Rev. X 10, 011053 (2020).

[213] W. Kress, U. Schröder, J. Prade, A. D. Kulkarni, and F. W. de Wette, Lattice dynamics of the high-Tc superconductor $\mathrm{YBa}_{2} \mathrm{Cu}_{3} \mathrm{O}_{7-x}$, Phys. Rev. B 38, 2906 (1988).

[214] R. Liu, C. Thomsen, W. Kress, M. Cardona, B. Gegenheimer, F. W. de Wette, J. Prade, A. D. Kulkarni, and U. Schröder, Frequencies, eigenvectors, and single-crystal selection rules of $\mathrm{k}=0$ phonons in $\mathrm{YBa}_{2} \mathrm{Cu}_{3} \mathrm{O}_{7-\delta}$ : Theory and experiment, Phys. Rev. B 37, 7971 (1988).

[215] D. Munzar, C. Bernhard, A. Golnik, J. Humlíčček, and M. Cardona, Anomalies of the infrared-active phonons in underdoped $\mathrm{YBa}_{2} \mathrm{Cu}_{3} \mathrm{O}_{y}$ as evidence for the intra-bilayer Josephson effect, Solid State Commun. 112, 365 (1999).

[216] C. C. Homes, T. Timusk, D. A. Bonn, R. Liang, and W. N. Hardy, Optical phonons polarized along the $\mathrm{c}$ axis of $\mathrm{YBa}_{2} \mathrm{Cu}_{3} \mathrm{O}_{6+x}$, for $\mathrm{x}=0.50 .95$, Can. J. Phys. 73, 663 (1995).

[217] T. P. Devereaux, T. Cuk, Z.-X. Shen, and N. Nagaosa, Anisotropic Electron-Phonon Interaction in the Cuprates, Phys. Rev. Lett. 93, 117004 (2004).

[218] S. Johnston, F. Vernay, B. Moritz, Z.-X. Shen, N. Nagaosa, J. Zaanen, and T. P. Devereaux, Systematic study of electronphonon coupling to oxygen modes across the cuprates, Phys. Rev. B 82, 064513 (2010).

[219] J. Humlíček, A. P. Litvinchuk, W. Kress, B. Lederle, C. Thomsen, M. Cardona, H.-U. Habermeier, I. E. Trofimov, and W. König, Lattice vibrations of $\mathrm{Y}_{1-x} \mathrm{Pr}_{x} \mathrm{Ba}_{2} \mathrm{Cu}_{3} \mathrm{O}_{7}$ : theory and experiment, Physica C 206, 345 (1993).

[220] R. Henn, T. Strach, E. Schönherr, and M. Cardona, Isotope effects on the optical phonons of $\mathrm{YBa}_{2} \mathrm{Cu}_{3} \mathrm{O}_{7}$ : Eigenvector and infrared charge determination, Phys. Rev. B 55, 3285 (1997).

[221] S. L. Cooper, D. Reznik, A. Kotz, M. A. Karlow, R. Liu, M. V. Klein, W. C. Lee, J. Giapintzakis, D. M. Ginsberg, B. W. Veal, and A. P. Paulikas, Optical studies of the $a$-, $b$-, and $c$-axis charge dynamics in $\mathrm{YBa}_{2} \mathrm{Cu}_{3} \mathrm{O}_{6+x}$, Phys. Rev. B 47, 8233 (1993).

[222] N. N. Kovaleva, A. V. Boris, T. Holden, C. Ulrich, B. Liang, C. T. Lin, B. Keimer, C. Bernhard, J. L. Tallon, D. Munzar, and A. M. Stoneham, $c$-axis lattice dynamics in Bi-based cuprate superconductors, Phys. Rev. B 69, 054511 (2004).

[223] We will only consider linear corrections in the phonon amplitude, and therefore we can treat the operator as a c-number for the purposes of the Schrieffer-Wolf transformation [197].
[224] Due to frustration at the mean-field level, the ground state remains invariant under in-plane rotations of the Néel vector, even though spin-rotation symmetry is explicitly broken by spin-orbit interactions. Therefore, whereas linear spin-wave theory predicts that one mode will remain gapless as a Goldstone mode, this is in fact a pseudo-Goldstone mode which must ultimately acquire a gap through an order-by-disorder mechanism.

[225] This definition of $\chi^{\text {diel }}$ is according to the notation used in, e.g., Jackson, and is not to be confused with the response function $\chi$ as defined in, e.g., Nozieres and Pines, which is actually the density-density response function.

[226] A. S. McLeod, P. Kelly, M. D. Goldflam, Z. Gainsforth, A. J. Westphal, G. Dominguez, M. H. Thiemens, M. M. Fogler, and D. N. Basov, Model for quantitative tip-enhanced spectroscopy and the extraction of nanoscale-resolved optical constants, Phys. Rev. B 90, 085136 (2014).

[227] F. Benz, M. K. Schmidt, A. Dreismann, R. Chikkaraddy, Y. Zhang, A. Demetriadou, C. Carnegie, H. Ohadi, B. d. Nijs, R. Esteban, J. Aizpurua, and J. J. Baumberg, Single-molecule optomechanics in "picocavities", Science 354, 726 (2016).

[228] C. Maissen, S. Chen, E. Nikulina, A. Govyadinov, and R. Hillenbrand, Probes for Ultrasensitive THz Nanoscopy, ACS Photon. 6, 1279 (2019).

[229] A. Cvitkovic, N. Ocelic, and R. Hillenbrand, Analytical model for quantitative prediction of material contrasts in scatteringtype near-field optical microscopy, Opt. Express 15, 8550 (2007).

[230] M. S. Anderson, Surface enhanced infrared absorption by coupling phonon and plasma resonance, Appl. Phys. Lett. 87, 144102 (2005).

[231] D. Isakov, C. J. Stevens, F. Castles, and P. S. Grant, A split ring resonator dielectric probe for near-field dielectric imaging, Sci. Rep. 7, 2038 (2017).

[232] X. Chen, X. Liu, X. Guo, S. Chen, H. Hu, E. Nikulina, X. Ye, Z. Yao, H. A. Bechtel, M. C. Martin, G. L. Carr, Q. Dai, S. Zhuang, Q. Hu, Y. Zhu, R. Hillenbrand, M. Liu, and G. You, THz near-field imaging of extreme subwavelength metal structures, ACS Photonics 7, 687 (2020).

[233] C. Chen, N. Hayazawa, and S. Kawata, A $1.7 \mathrm{~nm}$ resolution chemical analysis of carbon nanotubes by tip-enhanced Raman imaging in the ambient, Nat. Commun. 5, 3312 (2014).

[234] H.-T. Chen, R. Kersting, and G. C. Cho, Terahertz imaging with nanometer resolution, Appl. Phys. Lett. 83, 3009 (2003).

[235] C.-L. Yang, C.-S. Lee, K.-W. Chen, and K.-Z. Chen, Noncontact measurement of complex permittivity and thickness by using planar resonators, IEEE Trans. Microwave Theory Tech. 64, 247 (2016).

[236] G. Duan, J. Schalch, X. Zhao, A. Li, C. Chen, R. D. Averitt, and X. Zhang, A survey of theoretical models for terahertz electromagnetic metamaterial absorbers, Sensors Actuators A: Physical 287, 21 (2019).

[237] S. Bagiante, F. Enderli, J. Fabiańska, H. Sigg, and T. Feurer, Giant electric field enhancement in split ring resonators featuring nanometer-sized gaps, Sci. Rep. 5, 8051 (2015).

[238] Actually, it is not exactly equal to the bulk magnon resonance due to contributions from finite momentum magnons. It nevertheless remains close, at least for the parameters chosen here.

[239] M. H. Michael, A. von Hoegen, M. Fechner, M. Först, A Cavalleri, and E. Demler, Parametric resonance of Josephson 
plasma waves: A theory for optically amplified interlayer superconductivity in $\mathrm{YBa}_{2} \mathrm{Cu}_{3} \mathrm{O}_{6+x}$, Phys. Rev. B 102, 174505 (2020).

[240] In principle, one should also include other effects such as those implied by the full Rashba bilayer model, spin-wave broadening due to interactions or impurities, or the effects of weak $c$-axis exchange. The fate of the Van Hove singularity upon including all of these effects is clearly more complicated, albeit interesting and relevant to future studies.

[241] S. Sachdev, A. V. Chubukov, and A. Sokol, Crossover and scaling in a nearly antiferromagnetic fermi liquid in two dimensions, Phys. Rev. B 51, 14874 (1995).

[242] M. Le Tacon, G. Ghiringhelli, J. Chaloupka, M. M. Sala, V. Hinkov, M. W. Haverkort, M. Minola, M. Bakr, K. J. Zhou, S. Blanco-Canosa, C. Monney, Y. T. Song, G. L. Sun, C. T. Lin, G. M. De Luca, M. Salluzzo, G. Khaliullin, T. Schmitt, L. Braicovich, and B. Keimer, Intense paramagnon excitations in a large family of high-temperature superconductors, Nat. Phys. 7, 725 (2011).

[243] H. Sakai, J. Fujioka, T. Fukuda, M. S. Bahramy, D. Okuyama, R. Arita, T. Arima, A. Q. R. Baron, Y. Taguchi, and Y. Tokura, Soft phonon mode coupled with antiferromagnetic order in incipient-ferroelectric Mott insulators $\mathrm{Sr}_{1-x} \mathrm{Ba}_{x} \mathrm{MnO}_{3}$, Phys. Rev. B 86, 104407 (2012).

[244] E. Berg, E. Fradkin, and S. A. Kivelson, Theory of the striped superconductor, Phys. Rev. B 79, 064515 (2009).

[245] P. W. Anderson, Ten years of superconductivity: 1980-1990, Perspectives Condens. Matter Phys. 278 (1993).

[246] G. Ji, M. Xu, L. H. Kendrick, C. S. Chiu, J. C. Brüggenjürgen, D. Greif, A. Bohrdt, F. Grusdt, E. Demler, M. Lebrat, and M. Greiner, Coupling a Mobile Hole to an Antiferromagnetic Spin Background: Transient Dynamics of a Magnetic Polaron, Phys. Rev. X 11, 021022 (2021).

[247] C.-J. Kang and G. Kotliar, Optical Properties of the InfiniteLayer $\mathrm{La}_{1-x} \mathrm{Sr}_{x} \mathrm{NiO}_{2}$ and Hidden Hund's Physics, Phys. Rev. Lett. 126, 127401 (2021).

[248] J. Krishna, H. LaBollita, A. O. Fumega, V. Pardo, and A. S. Botana, Effects of Sr doping on the electronic and spinstate properties of infinite-layer nickelates: Nature of holes, Phys. Rev. B 102, 224506 (2020).

[249] M. Jiang, M. Berciu, and G. A. Sawatzky, Critical Nature of the Ni Spin State in Doped $\mathrm{NdNiO}_{2}$, Phys. Rev. Lett. 124, 207004 (2020).

[250] H. Sakakibara, H. Usui, K. Suzuki, T. Kotani, H. Aoki, and K. Kuroki, Model Construction and a Possibility of Cupratelike Pairing in a New $d^{9}$ Nickelate Superconductor (Nd,Sr) $\mathrm{NiO}_{2}$, Phys. Rev. Lett. 125, 077003 (2020).

[251] D. Li, K. Lee, B. Y. Wang, M. Osada, S. Crossley, H. R. Lee, Y. Cui, Y. Hikita, and H. Y. Hwang, Superconductivity in an infinite-layer nickelate, Nature (London) 572, 624 (2019).

[252] D. M. Juraschek and P. Narang, Highly Confined Phonon Polaritons in Monolayers of Perovskite Oxides, Nano Lett. 21, 5098 (2021).

[253] F. Schrodi, A. Aperis, and P. M. Oppeneer, Multichannel superconductivity of monolayer $\mathrm{FeSe}$ on $\mathrm{SrTiO}_{3}$ : Interplay of spin fluctuations and electron-phonon interaction, Phys. Rev. B 102, 180501(R) (2020).

[254] J. Paglione and R. L. Greene, High-temperature superconductivity in iron-based materials, Nat. Phys. 6, 645 (2010).
[255] L. Savary and L. Balents, Quantum spin liquids: a review, Rep. Prog. Phys. 80, 016502 (2017).

[256] P. Czajka, T. Gao, M. Hirschberger, P. Lampen-Kelley, A. Banerjee, J. Yan, D. G. Mandrus, S. E. Nagler, and N. P. Ong, Oscillations of the thermal conductivity in the spin-liquid state of $\alpha-\mathrm{RuCl}_{3}$, Nat. Phys. 17, 915 (2021).

[257] B. J. Kim, H. Ohsumi, T. Komesu, S. Sakai, T. Morita, H. Takagi, and T. Arima, Phase-Sensitive Observation of a SpinOrbital Mott State in $\mathrm{Sr}_{2} \mathrm{IrO}_{4}$, Science 323, 1329 (2009).

[258] B. J. Kim, H. Jin, S. J. Moon, J.-Y. Kim, B.-G. Park, C. S. Leem, J. Yu, T. W. Noh, C. Kim, S.-J. Oh, J.-H. Park, V. Durairaj, G. Cao, and E. Rotenberg, Novel $\mathrm{J}_{\text {eff }}=1 / 2$ Mott State Induced by Relativistic Spin-Orbit Coupling in $\mathrm{Sr}_{2} \mathrm{IrO}_{4}$, Phys. Rev. Lett. 101, 076402 (2008).

[259] J. Bertinshaw, Y. K. Kim, G. Khaliullin, B. J. Kim, Square lattice iridates, Annu. Rev. Condens. Matter Phys. 10, 315 (2019)

[260] T. Sarkar, D. S. Wei, J. Zhang, N. R. Poniatowski, P. R. Mandal, A. Kapitulnik, and R. L. Greene, Ferromagnetic order beyond the superconducting dome in a cuprate superconductor, Science 368, 532 (2020).

[261] N. P. Armitage, P. Fournier, and R. L. Greene, Progress and perspectives on electron-doped cuprates, Rev. Mod. Phys. 82, 2421 (2010).

[262] R. L. Greene, P. R. Mandal, N. R. Poniatowski, and T. Sarkar, The strange metal state of the electron-doped cuprates, Annu. Rev. Condens. Matter Phys. 11, 213 (2020).

[263] N. R. Poniatowski, T. Sarkar, S. Das Sarma, and R. L. Greene, Resistivity saturation in an electron-doped cuprate, Phys. Rev. B 103, L020501 (2021).

[264] T. Sarkar, N. R. Poniatowski, J. S. Higgins, P. R. Mandal, M. K. Chan, and R. L. Greene, Hidden strange metallic state in underdoped electron-doped cuprates, Phys. Rev. B 103, 224501 (2021).

[265] Y. Cao, V. Fatemi, A. Demir, S. Fang, S. L. Tomarken, J. Y. Luo, J. D. Sanchez-Yamagishi, K. Watanabe, T. Taniguchi, E. Kaxiras, R. C. Ashoori, and P. Jarillo-Herrero, Correlated insulator behaviour at half-filling in magic-angle graphene superlattices, Nature (London) 556, 80 (2018).

[266] M. Vogl, M. Rodriguez-Vega, B. Flebus, A. H. MacDonald, and G. A. Fiete, Floquet engineering of topological transitions in a twisted transition metal dichalcogenide homobilayer, Phys. Rev. B 103, 014310 (2021).

[267] S. Chatterjee, P. E. Dolgirev, I. Esterlis, A. A. Zibrov, M. D. Lukin, N. Y. Yao, and E. Demler, Single-spin qubit magnetic spectroscopy of two-dimensional superconductivity, Phys. Rev. Research 4, L012001 (2022).

[268] N. R. Poniatowski, J. B. Curtis, A. Yacoby, and P. Narang, Spectroscopic signatures of time-reversal symmetry breaking superconductivity, arXiv:2103.05641.

[269] J. F. Rodriguez-Nieva, K. Agarwal, T. Giamarchi, B. I. Halperin, M. D. Lukin, and E. Demler, Probing onedimensional systems via noise magnetometry with single spin qubits, Phys. Rev. B 98, 195433 (2018).

[270] S. Chatterjee, J. F. Rodriguez-Nieva, and E. Demler, Diagnosing phases of magnetic insulators via noise magnetometry with spin qubits, Phys. Rev. B 99, 104425 (2019).

[271] M. Zeng, L.-H. Hu, H.-Y. Hu, Y.-Z. You, and C. Wu, Phase-fluctuation Induced Time-Reversal Symmetry Breaking Normal State, arXiv:2102.06158. 
[272] V. Grinenko, D. Weston, F. Caglieris, C. Wuttke, C. Hess, T. Gottschall, J. Wosnitza, A. Rydh, K. Kihou, C.-H. Lee, R. Sarkar, S. Dengre, I. Maccari, J. Garaud, A. Charnukha, R. Hühne, K. Nielsch, B. Büchner, H.-H. Klauss, and E. Babaev, State with spontaneously broken time-reversal symmetry above the superconducting phase transition, Nat. Phys. 17, 1254 (2021).
[273] Z. Viskadourakis, S. S. Sunku, S. Mukherjee, B. M. Andersen, T. Ito, T. Sasagawa, and C. Panagopoulos, Ferroelectricity in underdoped La-based cuprates, Sci. Rep. 5, 15268 (2015).

[274] S. Mukherjee, B. M. Andersen, Z. Viskadourakis, I. Radulov, and C. Panagopoulos, Theory of the magnetoeletric effect in a lightly doped high-Tc cuprate, Phys. Rev. B 85, 140405(R) (2012). 